\title{
Aplicações da teoria de Bases de Gröbner para o cálculo da cohomologia de Hochschild.
}

Ana Melisa Paiba Amaya

\author{
DisSERTAÇÃO APRESENTADA \\ $\mathrm{AO}$ \\ Instituto De Matemática e EstatísticA \\ DA \\ Universidade DE SÃo PAUlo \\ PARA \\ OBTENÇÃO DO TÍTULO \\ $\mathrm{DE}$ \\ Mestre em CIÊNCIAS
}

Programa: Matemática

Orientador: Prof. Dr. Eduardo do Nascimento Marcos

Durante o desenvolvimento deste trabalho a autora recebeu auxílio financeiro da FAPESP

São Paulo, dezembro de 2018 


\section{Aplicações da teoria de Bases de Gröbner para o cálculo da cohomologia de Hochschild.}

Esta versão da dissertação contém as correções e alterações sugeridas pela Comissão Julgadora durante a defesa da versão original do trabalho, realizada em 24/10/2018. Uma cópia da versão original está disponível no

Instituto de Matemática e Estatística da Universidade de São Paulo.

Comissão Julgadora:

- Prof. Dr. Eduardo do Nascimento Marcos (orientador) - IME-USP

- Prof. Dra. Regina Maria de Aquino - UFES

- Prof. Dr. Viktor Bekkert - UFMG 


\section{Agradecimentos}

Primeiramente, agradeço ao meu orientador, o professor Eduardo do Nascimento Marcos pela sua excelente orientação. O trabalho junto com ele é muito motivante . Ele é um ótimo matemático que com seus conhecimentos e compreensão facilitou o desenvolvimento desta dissertação e a minha estadia aqui em São Paulo. Eu me sinto muito grata para com ele e acho que eu não poderia ter um melhor orientador do que ele.

Agradeço aos meus pais Hector Paiba e Martha Amaya por seu amor e esforço colocado em mim e pelo qual eu consegui alcançar as minhas metas até agora. Aos meus irmãos, por ser a minha motivação diária para continuar trabalhando no meu crescimento pessoal e acadêmico. E agradeço a Raúl, meu companheiro e suporte quem esteve comigo em todo momento nos últimos anos.

Agradeço a Alex Sierra pela sua disponibilidade para esclarecer as minhas dúvidas relacionadas à dissertação.

Agradeço à Fundação de apoio à pesquisa do estado de São Paulo (FAPESP) pelo suporte financeiro (Processo FAPESP no 2016/09809-5). As opiniões, hipóteses e conclusões ou recomendações expressas neste material são de responsabilidade da autora e não necessariamente refletem a visão da FAPESP. 


\section{Resumo}

\section{PAIBA, A. M. Aplicações da teoria de Bases de Gröbner para o cálculo da cohomologia}

de Hochschild. 2018. 46 f. Dissertação (Mestrado) - Instituto de Matemática e Estatística, Universidade de São Paulo, São Paulo, 2018.

A Cohomologia de Hochschild é um invariante associado a álgebras o qual pode nos fornecer propiedades homologicas das álgebras e suas categorias de módulos. Além disso tem aplicações em Geometria Algébrica e Teoria de Representações, entre outras áreas. Para álgebras $A$ sobre um corpo, o $i$-ésimo grupo de cohomologia de Hochschild $H H^{i}(A, M)$ de $A$, com coeficientes no bimódulo $M$, coincide com $\operatorname{Ext}_{A^{e}}^{i}(A, M)$. Logo, este pode ser calculado usando uma resolução projetiva da álgebra como $A$-bimódulo. Diferentes autores como Dieter Happel, Claude Cibils, Edward Green, David Anick, Michael Bardzell e Andrea Solotar desenvolveram ferramentas para a construção destas resoluções em casos específicos [Hap89, Cib90, Bar97, AG87]. Um resultado recente e muito importante é apresentado por Andrea Solotar e Sergio Chohuy [CS15], onde se mostra a construção de uma resolução projetiva de bimódulos para álgebras associativas generalizando o resultado para álgebras monomiais feito por Bardzell [Bar97]. Nesta dissertação pretendemos introduzir ao leitor no conceito de Cohomologia de Hochschild mostrando a importância da mesma mediante resultados conhecidos para álgebras de dimensão finita. Além disso, apresentamos os conceitos e resultados do trabalho de Chohuy e Solotar mencionado acima. No decorrer deste trabalho complementamos algumas demonstrações dos resultados enunciados com o fim de propiciar uma ferramenta para o melhor entendimento dos tópicos trabalhados aqui.

Palavras-chave: Cohomologia de Hochschild, álgebras associativas, resolução projetiva, sistemas de redução. 


\section{Abstract}

\section{PAIBA, A. M. Aplicações da teoria de Bases de Gröbner para o cálculo da cohomologia}

de Hochschild. 2018. 46 f. Dissertação (Mestrado) - Instituto de Matemática e Estatística, Universidade de São Paulo, São Paulo, 2018.

The Hochschild Cohomology is an invariant attached to associative algebras which may provide us some homological aspects of the algebras and its category of modules. Moreover, it has applications to Algebraic Geometry and Representation Theory, among others areas. For algebras $A$ over a field the Hochschild cohomology group $H H^{i}(A, M)$ of $A$ with coeficients in a bimodule $M$ coincides with $\operatorname{Ext}_{A^{e}}^{i}(A, M)$. So it can be computed using a projective resolution of the algebra, as a bimodule over itself. Therefore different authors like Dieter Happel, Claude Cibils, Edward Green, David Anick, Michael Bardzell, Sergio Chohuy and Andrea Solotar developed tools for the construction of these resolutions in particular cases [Hap89, Cib90, Bar97, AG87]. A recent and very important result was introduced by Andrea Solotar and Sergio Chohuy [CS15], where they show a construction of a projective bimodule resolution for associative algebras generalizing the result for monomial algebras made by Bardzell [Bar97]. In this dissertation we intend to introduce the reader in the cohomology Hochschild concept, showing its importance through known results for finite dimensional algebras. Besides, we exhibit the concepts and results of Chohuy and Solotar mentioned before. During this text, we complement some demonstrations with the purpose of giving a tool for the a better understanding.

Keywords: Hochschild cohomology, associative algebras, projective resolution, reduction system. 


\section{Sumário}

$\begin{array}{ll}\text { Introdução } & 1\end{array}$

1 Álgebras $\quad 3$

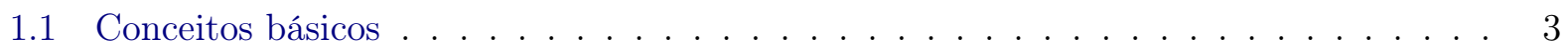

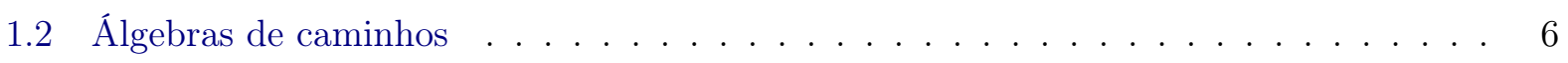

1.3 Teorema de Gabriel . . . . . . . . . . . . . . . . . . . . . . 8

2 Cohomologia de Hochschild $\quad 11$

2.1 Definição de cohomologia de Hochschild . . . . . . . . . . . . . . . . . 11

2.2 Interpretação dos primeiros grupos de cohomologia . . . . . . . . . . . . . . . . 16

$2.2 .1 \quad$ 0-ésimo grupo de cohomologia . . . . . . . . . . . . . . . . . . 16

2.2 .2 Primeiro grupo de cohomologia . . . . . . . . . . . . . . . 16

2.2 .3 Segundo grupo de cohomologia . . . . . . . . . . . . . . . . . 18

2.3 Álgebras hereditárias . . . . . . . . . . . . . . . . . . . . . . . . 20

2.4 Carcases estreitos . . . . . . . . . . . . . . . . . . . . 21

2.5 Álgebras de radical quadrado zero . . . . . . . . . . . . . . . . . . . 22

3 Resolução projetiva para Álgebras associativas $\quad \mathbf{2 7}$

3.1 Sistemas de Redução . . . . . . . . . . . . . . . . . . . . . . 27

3.1.1 Construção de um sistema de redução que satisfaz a condição do Diamante 31

3.2 Ambiguidades . . . . . . . . . . . . . . . . . . . . 33

3.2 .1 Álgebras monomiais . . . . . . . . . . . . . . . . . . . . 34

3.3 Resolução projetiva . . . . . . . . . . . . . . . . . . . . . . 35

3.4 Primeiros homomorfismos da resolução . . . . . . . . . . . . . . . . . . . . . . 43

3.5 Contra exemplo da questão de Happel . . . . . . . . . . . . . . . . . . . . . . 45

$\begin{array}{ll}\text { Considerações finais } & 51\end{array}$

$\begin{array}{ll}\text { Referências Bibliográficas } & 53\end{array}$ 


\section{Introdução}

A Cohomologia de Hochschild é um invariante por equivalências derivadas associados a álgebras associativas que além de fornecer informações importantes de tipos particulares de álgebras tem conexão com outras áreas da matemática como Geometria Algébrica e Teoria de Representações de Álgebras de dimensão finita[Ger64]. O conceito de Cohomologia de Hochschild foi introduzido no ano 1945 por Hochschild, quem ao mesmo tempo faz uso desta cohomologia para mostrar uma condição necessária e suficiente para uma álgebra $A$ ser separável, e além disso proporciona uma interpretação dos primeiros grupos de cohomologia [Hoc45].

Uma outra abordagem dos grupos de cohomologia no caso em que $A$ é uma álgebra sobre um corpo $k$, está baseada na aplicação do funtor $\operatorname{Hom}(-, M)$, com $M$ um $A$-bimódulo, à resolução padrão [CE16]. Isto faz com que as resoluções projetivas de $A$ sobre a sua álgebra envolvente $A^{e}:=A^{o p} \otimes_{k} A$, ou equivalentemente, resoluções projetivas de $A$ como $A$-bimódulo sejam uma ferramenta útil para o cálculo dos grupos de cohomologia de Hochschild. Para o caso de álgebras associativas de dimensão finita Happel fornece os módulos projetivos de uma resolução projetiva minimal, sem definir os homomorfismos [Hap89]. Em [Cib90] Cibils define uma resolução no caso em que $A / \mathrm{radA}$ é uma álgebra separável. Bardzell exibe uma resolução de bimódulos para álgebras monomiais, isto é, álgebras da forma $A=k Q / I$ com $k$ um corpo, $Q$ um carcás finito e $I$ um ideal bilateral gerado por relações monomiais [Bar97]. Resultados para casos mais gerais (álgebras não monomiais) foram obtidos por Anick e Green [Ani86][AG87]. Um trabalho recente que generaliza o feito por Bardzell e que faz uso das ideias da Teoria de Bases de Gröbner é o trabalho feito por Chohuy e Solotar, onde é calculada de maneira indutiva uma resolução projetiva para álgebras (não necessariamente de dimensão finita) da forma $k Q / I$, com $Q$ um carcás com um número finito de vértices e $I$ um ideal bilateral de $k Q$. Para isto, os projetivos e os homomorfismos da resolução são definidos mediantes $n$-ambiguidades construídas a partir de um sistema de redução $\mathcal{R}$ que satisfaz a condição do Diamante para $I$ [CS15].

Nesta dissertação pretendemos introduzir ao leitor ao conceito de Cohomologia de Hochschild, assim como mostrar alguns resultados conhecidos da teoria comentados acima. Faremos enfase no resultado recente de Chohuy e Solotar [CS15], a qual é uma ferramenta que abre caminho a novos resultados importantes na área.

No Capítulo 1 apresentamos os conceitos e resultados básicos sobre álgebras e álgebras de caminhos a serem usados ao decorrer do trabalho. Para o caso de álgebras de dimensão finita sobre um corpo $k$ algebricamente fechado enunciamos o Teorema de Gabriel, o qual joga um papel importante por causa da cohomologia de Hochschild ser invariante por categorias derivadas, em particular, por equivalência Morita.

No Capítulo 2 definimos a Cohomologia de Hochschild junto com a construção desta a partir da resolução estândar. Mostramos a interpretação dos primeiros grupos de cohomologia e apresentamos resoluções projetiva que permitem calcular os grupos de cohomologia de Hochschild para tipos particulares de álgebras como álgebras hereditárias e álgebras de radical quadrado zero.

No Capítulo 3 apresentamos os conceitos relacionados com sistemas de redução associados aos 
geradores de um ideal. Dado um ideal bilateral $I$ de $k Q$ mostramos que sempre existe um sistema de redução que satisfaz a condição do Diamante para $I$. Dada uma ordem admissível sobre o conjunto dos caminhos $Q_{\geq 0}$ mostramos um método para construir um sistema de redução que satisfaz a condição do Diamante para $I$, não tem ambiguidades de inclusão e toda ambiguidade é resolvível. Com um sistema de redução $\mathcal{R}$ como o construído definimos as $n$-ambiguidades, necessárias para a construção da resolução projetiva desejada. Finalmente construímos de maneira indutiva uma resolução projetiva de bimódulos para álgebras da forma $A=k Q / I$ onde o carcás $Q$ tem um número finito de vértices e $I$ é um ideal bilateral, e são dados explicitamente os primeiros homomorfismos da resolução. Essa resolução é obtida no trabalho de Chouhy e Solotar como uma generalização da resolução de Bardzell. Para o caso monomial as duas coincidem e nesse caso a resolução é minimal. No caso não monomial embora não seja minimal, ela é bem mais computável que a Resolução bar. 


\section{Capítulo 1}

\section{Álgebras}

Uma das propriedades mais importantes da cohomologia de Hochschild é o fato de ela ser invariante por equivalências derivadas, em particular por equivalência de Morita, ou seja, dadas duas $k$-álgebras $A$ e $B$ tal que as categorias $\bmod A$ e $\bmod B$ são equivalentes, então $H^{i}(A) \cong H^{i}(B)$ [Wei95]. Em particular, dado um corpo $k$ algebricamente fechado, e uma $k$-álgebra $A$ sempre existe uma álgebra básica Morita equivalente a $A$ [AF12], então podemos nos restringir ao cálculo de cohomologias de Hochschild de álgebras básicas. O objetivo deste capitulo é mostrar os conceitos básicos de álgebras e álgebras de caminhos a ser usados ao longo do texto, como também caracterizar as álgebras de dimensão finita básicas indecomponíveis como quocientes de álgebras de caminhos por um ideal admissível.

As demonstrações dos resultados enunciados neste capitulo podem ser encontradas em [ASS06] , [CLS82] ou em qualquer texto de Teoria de Representações de Álgebras.

\subsection{Conceitos básicos}

Definição 1. Seja $k$ um corpo. Uma k-álgebra é um anel $A$ com elemento identidade, tal que $A$ tem estrutura de $k$-espaço vetorial e além disso

$$
\lambda(a b)=(a \lambda) b=a(\lambda b)=(a b) \lambda
$$

para todo $\lambda \in k$ e todo $a, b \in A$. Dizemos que $A$ é uma álgebra de dimensão finita se $A$ é de dimensão finita como $k$ - espaço vetorial.

Dadas $A$ e $B$ duas $k$-álgebras, um homomorfismo de anéis $f: A \rightarrow B$ é um homomorfismo de $k$-álgebras se $f$ é uma aplicação $k$-linear.

Ao longo deste texto $k$ denotará um corpo, a menos de menção em contrário.

Exemplo 1. (1) O anel de polinômios em $n$ variáveis com coeficientes em $k, k\left[x_{1}, \ldots, x_{n}\right]$ é uma k-álgebra de dimensão infinita.

(2) O anel de polinômios em $n$ variáveis não comutativas, $k\left\langle x_{1}, \ldots, x_{n}\right\rangle$ é uma $k$-álgebra de dimensão infinita tal que

$$
k\left\langle x_{1}, \ldots, x_{n}\right\rangle / I \cong k\left[x_{1}, \ldots, x_{n}\right]
$$

com I o ideal bilateral gerado por $\left\{x_{i} x_{j}-x_{j} x_{i} \mid i, j=1, \ldots, n\right\}$.

(3) Dada uma k-álgebra $A$ e $n \in \mathbb{N}$, o conjunto das matrizes $n \times n$ com coeficientes em $A$, notada por $\mathbb{M}_{n}(A)$ é uma $k$-álgebra ( com a soma e produto usual de matrizes) de dimensão finita. 
(4) Como é feito para toda estrutura algébrica, podemos definir o conceito de subálgebra como um subconjunto de uma álgebra A que mantém a estrutura de álgebra. Por exemplo, o conjunto das matrizes triangulares inferiores,

$$
L_{n}(K)=\left[\begin{array}{cccc}
k & 0 & \ldots & 0 \\
k & k & \ldots & 0 \\
\vdots & \vdots & \ddots & \vdots \\
k & k & \ldots & k
\end{array}\right]
$$

é uma k-subálgebra de $\mathbb{M}_{n}(K)$.

(5) Seja $(I, \preceq)$ um poset (conjunto parcialmente ordenado), onde $I=\left\{a_{1}, \ldots, a_{n}\right\}$ e $\preceq$ é uma relação binária de ordem parcial sobre I. Seja

$$
K I=\left\{\left[\lambda_{i j}\right] \in \mathbb{M}_{n}(k) \mid \lambda_{s t}=0 \quad \text { se } \quad a_{s} \npreceq a_{t}\right\}
$$

isto é, o conjunto formado pelas matrizes $\left[\lambda_{i j}\right]$ tal que $\lambda_{i j}=0$ se a relação $a_{i} \preceq a_{j}$ não se satisfaz em $I$.

$K I$ é uma $k$-subálgebra de $\mathbb{M}_{n}(k)$ chamada álgebra de incidência do poset $(I, \preceq)$ com coeficientes em $k$. As matrizes $\left\{e_{i j}\right\}$ com $a_{i} \preceq a_{j}$ formam uma base do $k$-espaço vetorial $K I$.

(6) Para toda k-álgebra A definimos a sua álgebra oposta $A^{o p}$ como a k-álgebra cuja estrutura de espaço vetorial subjacente é a mesma de A, mas com o produto $*$ em $A^{\text {op }}$ definido por $a * b=b a$.

Notação: vamos notar por $\operatorname{Mod} A$ a categoria dos $A$-módulos à direita, e vamos notar por $\bmod A$ a subcategoria plena de $\operatorname{Mod} A$ formada pelos módulos finitamente gerados. Se $A$ é uma álgebra de dimensão finita, $\bmod A$ coincide com a subcategoria plena de $\operatorname{Mod} A$ formada pelos módulos de $k$-dimensão finita.

Definição 2. Um A-módulo não nulo $M$ é dito indecomponível se $M$ não pode ser expresso como uma soma direta de submódulos não triviais.

A fim de estudar os módulos em modA os módulos indecomponíveis desempenham um papel importante já que basta conhecer os módulos indecomponíveis para construir qualquer módulo sobre $A$.

Teorema 1 (Krull-Schmidt). Seja A uma k-álgebra de dimensão finita. Todo módulo $M$ em modA tem uma única decomposição em soma direta de um número finito de módulos indecomponíveis em $\bmod A$. Isto é,

$$
M \cong M_{1} \oplus M_{2} \oplus \cdots \oplus M_{n}
$$

onde cada $M_{i}$ é indecomponivel, e se existe outra decomposição $M \cong N_{1} \oplus N_{2} \oplus \cdots \oplus N_{m}$ com cada $N_{i}$ indecomponivel, então $m=n$ e existe uma permutação $\sigma \in S_{n}$ tal que $M_{i} \cong N_{\sigma(i)}$ para todo $i$.

Podemos ver a álgebra $A$ como um objeto de $\bmod A$, então pelo anterior $A$ tem uma decomposição única em indecomponíveis de $\bmod A$

$$
A \cong P_{1} \oplus P_{2} \oplus \cdots \oplus P_{n}
$$

Cada um dos $P_{i}$ 's que aparece na decomposição de $A$ é um $A$-módulo projetivo indecomponível pois cada um deles é somando direto de um $A$-módulo livre (neste caso $A$ ). Usando o fato de todo projetivo ser somando direto de um $A$-módulo isomorfo a uma soma de copias do $A$-módulo $A$, e o Teorema de Krull-Schmidt podemos concluir que todo projetivo indecomponível é isomorfo a algum $\operatorname{dos} P_{i}$ 's que aparecem na decomposição do $A$. 
Definição 3. Seja $A$ uma k-álgebra e $\left\{e_{1}, \ldots, e_{n}\right\}$ um subconjunto de $A$. Dizemos que $\left\{e_{1}, \ldots, e_{n}\right\}$ é um sistema completo de idempotentes ortogonais primitivos se

(1) $\sum_{i=1}^{n} e_{i}=1$ (sistema completo),

(2) $e_{i}^{2}=e_{i}$ para todo $1 \leq i \leq n$ (elementos idempotentes),

(3) $e_{i} e_{j}=0$ se $i \neq j$ (elementos ortogonais),

(4) Se $e_{i}=f+g$ com $f$ e $g$ idempotentes ortogonais, então $f=0$ ou $g=0$.(elementos primitivos)

Dada uma $k$-álgebra $A$ e a sua decomposição em módulos indecomponíveis

$$
A \cong P_{1} \oplus P_{2} \oplus \cdots \oplus P_{n}
$$

podemos decompor o elemento identidade de $A$ como $1=e_{1}+\cdots+e_{n}$. O conjunto $\left\{e_{1}, \ldots, e_{n}\right\}$ resulta ser um sistema completo de idempotentes ortogonais primitivos de $A$. Reciprocamente, um subconjunto de $A$ satisfazendo as condições da Definição 3 fornece uma decomposição em indecomponíveis de $A$.

Proposição 1. Seja $A$ uma $k$-álgebra e $\left\{e_{1}, \ldots, e_{n}\right\}$ um sistema completo de idempotentes ortogonais primitivos de $A$, com $e_{i} \neq 0$ para todo $i$. Então

$$
A=e_{1} A \oplus e_{2} A \oplus \cdots \oplus e_{n} A
$$

é uma decomposição de A em módulos indecomponiveis.

Portanto, uma lista completa de classes de isomorfia de projetivos indecomponíveis em modA está dada por $e_{1} A, e_{2} A, \ldots, e_{n} A$.

Em seguida vamos dar a definição de álgebra básica e álgebra indecomponível junto com os resultados que garantem que podemos nos restringir ao estudo deste tipo de álgebras sem perder generalidade.

Definição 4. Uma k-álgebra $A$ de dimensão finita é dita básica se, na decomposição dada em (1.1), $e_{i} A \cong e_{j} A$ se e somente se $i=j$.

Teorema 2. Dada A uma k-álgebra de dimensão finita, existe uma única (a menos de isomorfismo) $k$-álgebra $A^{\prime}$ de dimensão finita básica tal que $\bmod A$ e $\bmod A^{\prime}$ são categorias equivalentes.

No caso em que dados dois anéis $A$ e $B$ as categorias $\bmod A$ e $\bmod B$ são equivalentes, dizemos que $A$ e $B$ são Morita equivalentes. Logo, pelo teorema anterior, dada uma $k$-álgebra de dimensão finita, existe uma $k$-álgebra de dimensão finita básica Morita equivalente a ela.

Definição 5. Uma k-álgebra e chamada indecomponivel se não pode ser expressa como soma direta de duas $k$-álgebras, ou equivalentemente, se seus únicos idempotentes centrais são 0 e 1 , ou seja, se existe $e \in A$ tal que $e^{2}=e$ e ex $=x e$ para todo $x \in A$, então $e=1$ ou $e=0$.

Proposição 2. Toda k-álgebra de dimensão finita é isomorfa a uma soma direta de um número finito de k-álgebras indecomponíveis de dimensão finita.

Proposição 3. Se $A=\prod_{i=1}^{n} A_{i}$, então $\bmod A \cong \prod_{i=1}^{n} \bmod A_{i}$.

Definição 6. O radical ou radical de Jacobson de uma k-álgebra $A$ é a interseção de todos os ideais à direita maximais de A. Este ideal é um ideal bilateral e denotamos este ideal por radA.

Nota: Equivalentemente, podemos definir $\operatorname{rad} A$ como a interseção de todos os ideais à esquerda maximais de $A$.

Proposição 4. Seja A uma k-álgebra. Então A/radA é uma k-álgebra de radical zero.

Proposição 5. Seja A uma k-álgebra. Se I é um ideal bilateral nilpotente de $A$, ou seja, $I^{m}=0$ para algum $m \geq 1$, então $I \subseteq$ radA. Se além disso, $A / I$ é isomorfo a um produto de copias de $k$, então $I=\operatorname{rad} A$. 


\section{2 Álgebras de caminhos}

Definição 7. Um carcás $Q=\left(Q_{0}, Q_{1}, o, t\right)$ é uma quádrupla formada por um conjunto de vértices $Q_{0}$, um conjunto de flechas $Q_{1}$, e duas aplicações $o, t: Q_{1} \rightarrow Q_{0}$ que associam a cada flecha $\alpha \in Q_{1}$ seu origem $o(\alpha) \in Q_{0}$ e seu término $t(\alpha) \in Q_{0}$ respectivamente.

Um carcás $Q$ é finito se $Q_{0}$ e $Q_{1}$ são conjuntos finitos e é conexo se o grafo subjacente é conexo.

Notamos por $\alpha: v \rightarrow v^{\prime}$ a flecha $\alpha \in Q_{1}$ com origem $v=o(\alpha)$ e término $v^{\prime}=t(\alpha)$. O carcás $Q=\left(Q_{0}, Q_{1}, o, t\right)$ é usualmente notado por $Q=\left(Q_{0}, Q_{1}\right)$ ou simplesmente $Q$.

Exemplo 2. Os seguintes são exemplos de carcases

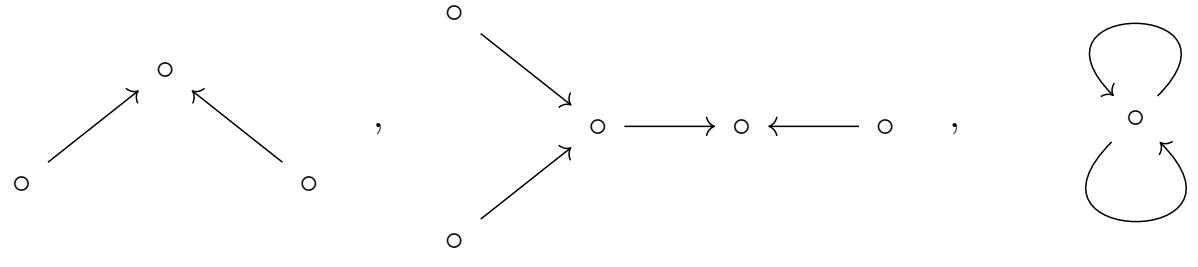

Definição 8. Seja $Q$ um carcás e $v, v^{\prime} \in Q_{0}$. Um caminho sobre $Q$ de comprimento $l \geq 1$ com origem $v$ e término $v^{\prime}$ é uma sequência

$$
\gamma=\alpha_{1} \alpha_{2} \cdots \alpha_{l}
$$

tal que $\alpha_{i} \in Q_{1}$ para todo $1 \leq i \leq l$ e o $\left(\alpha_{1}\right)=v, o\left(\alpha_{2}\right)=t\left(\alpha_{1}\right), \ldots, o\left(\alpha_{l}\right)=t\left(\alpha_{l-1}\right), t\left(\alpha_{l}\right)=v^{\prime}$.

Notamos por $Q_{l}$ o conjunto dos caminhos em $Q$ de comprimento $l$. Adicionalmente, associamos a cada vértice $v_{i} \in Q_{0}$ um caminho de comprimento $l=0$, denominado o caminho trivial em $v_{i}$, e notado por $\tau_{i}$.

Definição 9. Um caminho de comprimento $l \geq 1$ é chamado um ciclo se seu origem e término coincidem. Se o comprimento de um ciclo é 1, então dizemos que ele é um laço.

Definição 10. Se existe em $Q$ um caminho de $v$ a $v^{\prime}$, então $v$ é dito um antecessor de $v^{\prime}$, e $v^{\prime}$ é dito um sucessor de $v$.

Dado um carcás $Q$ e um corpo $k$ é possível construir uma $k$-álgebra definindo o produto de caminhos como segue.

Definição 11. Seja $Q$ um carcás. A álgebra de caminhos $k Q$ é a k-álgebra cujo k-espaço vetorial subjacente tem como base o conjunto de todos os caminhos de comprimento $l \geq 0$ em $Q$. O produto de dois caminhos da base $\gamma_{1}=\alpha_{1} \alpha_{2} \cdots \alpha_{s}$ e $\gamma_{2}=\beta_{1} \beta_{2} \cdots \beta_{t}$ está dado por:

$$
\gamma_{1} \gamma_{2}= \begin{cases}\alpha_{1} \alpha_{2} \cdots \alpha_{s} \beta_{1} \beta_{2} \cdots \beta_{t} & \text { se } t\left(\alpha_{s}\right)=o\left(\beta_{1}\right) \\ 0 & \text { se } t\left(\alpha_{s}\right) \neq o\left(\beta_{1}\right)\end{cases}
$$

Já definido o produto sobre os elementos da base podemos estender o produto a todos os elementos de $k Q$ por linearidade.

Definição 12. Seja $Q$ um carcás e $\gamma_{1}, \gamma_{2}$ caminhos sobre $Q$. Dizemos que $\gamma_{2}$ divide a $\gamma_{1}$ se existem caminhos a e b de comprimento maior ou igual a zero tais que $\gamma_{1}=a \gamma_{2} b$.

Definição 13. Dado um carcás $Q$ e $\gamma_{1}, \gamma_{2}$ caminhos sobre $Q$ dizemos que $\gamma_{1}$ e $\gamma_{2}$ são caminhos paralelos se $o\left(\gamma_{1}\right)=o\left(\gamma_{2}\right)$ e $t\left(\gamma_{1}\right)=t\left(\gamma_{2}\right)$.

Agora vamos apresentar alguns resultados importantes sobre as álgebras de caminhos 
Lema 1. Seja $Q$ um carcás e $k Q$ sua álgebra de caminhos. Então

(1) $k Q$ é uma álgebra associativa,

(2) $k Q$ tem um elemento identidade se e somente se $Q_{0}$ é finito,

(3) $k Q$ tem dimensão finita se e somente se $Q$ é finito e não tem ciclos.

Se $Q$ é um carcás finito e conexo, a álgebra de caminhos $k Q$ é uma $k$-álgebra associativa indecomponível com identidade, e com $\left\{\tau_{i} \mid v_{i} \in Q_{0}\right\}$ sendo um sistema completo de idempotentes ortogonais primitivos.

Daqui em diante, dado um carcás finito e conexo denotaremos por $F$ o ideal bilateral de $k Q$ gerado pelas flechas de $Q$. Note que $F$ coincide com o $k$-espaço vetorial de $k Q$ que tem por base os caminhos de comprimento $l \geq 1$.

Proposição 6. Seja $Q$ um carcás finito e conexo e seja $k Q$ a sua álgebra de caminhos. Se $Q$ não tem ciclos, o radical de $k Q$ coincide com $F$.

Se $Q$ tem algum ciclo o anterior pode não valer. Por exemplo, seja $Q$ o seguinte carcás :

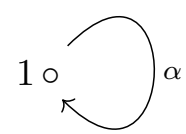

Uma base de $k Q$ está dada por $\left\{\tau_{1}, \alpha, \alpha^{2}, \ldots, \alpha^{t}, \ldots\right\}$ e o produto de dois elementos da base vai estar definido por $\tau_{1} \alpha^{t}=\alpha^{t} \tau_{1}=\alpha^{t}$ para todo $t \geq 1$, e $\alpha^{t} \alpha^{s}=\alpha^{t+s}$ para quaisquer $t, s \geq 1$. Portanto, $k Q$ é isomorfa a álgebra de polinômios $K[x]$ e $\operatorname{rad}(k Q)=0$.

Definição 14. Seja $Q$ um carcás finito e $F$ o ideal bilateral de $k Q$ gerado pelas flechas. Um ideal bilateral $I$ de $k Q$ é dito admissivel se $F^{m} \subseteq I \subseteq F^{2}$ para algum $m \geq 2$.

Se I é um ideal admissível de $k Q$, o par $(Q, I)$ é chamado um carcás limitado.

Exemplo 3. (1) Se $Q$ não tem ciclos, então todo ideal contido em $F^{2}$ é um ideal admissível.

(2) Dado um carcás $Q$ e $m \geq 2, F^{m}$ é admissível.

(3) O ideal zero é admissível em $k Q$ se e somente se $Q$ não tem ciclos.

(4) Seja $Q$ o seguinte carcás

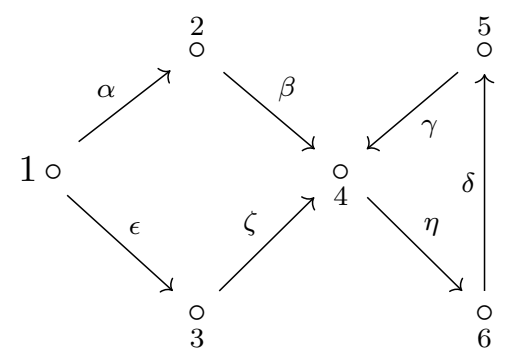

e seja I o ideal bilateral gerado por $\{\alpha \beta-\epsilon \zeta, \beta \eta, \eta \delta \gamma\}$. É claro que $I \subset F^{2}$. Por outro lado, $\eta \delta \gamma$ divide todo caminho $\Upsilon$ de comprimento maior ou igual a 5 com origem $1,3,4,5$ ou 6 , e em consequência $\Upsilon \in I$, e $\beta \eta$ divide todo caminho de comprimento maior o igual a 2 com origem em 2 , então estes caminhos também pertencem a I. Pelo anterior, $F^{5} \subset I$ e I resulta ser um ideal admissivel. 
Proposição 7. Seja $Q$ um carcás finito e I um ideal admissível de $k Q$. Então

(1) $O$ conjunto $\left\{\overline{\tau_{i}}=\tau_{i}+I \mid v_{i} \in Q_{0}\right\}$ é um sistema completo de idempotentes ortogonais primitivos da álgebra $k Q / I$.

(2) $k Q / I$ é indecomponivel se e somente se $Q$ é conexo.

(3) $k Q / I$ é uma álgebra de dimensão finita.

(4) $\operatorname{rad}(k Q / I)=F / I$.

(5) $k Q / I$ é uma álgebra básica.

É conveniente expressar um ideal admissível em termo dos seus geradores.

Definição 15. Seja $Q$ um carcás. Uma relação em $Q$ com coeficientes em $k$ é uma combinação $k$-linear de caminhos de comprimento maior ou igual a 2 , tais que seus origens coincidem e seus términos coincidem. Ou seja, uma relação $\rho$ é um elemento de $k Q$ tal que

$$
\rho=\sum_{i=1}^{n} \lambda_{i} \gamma_{i},
$$

onde $0 \neq \lambda_{i} \in k$ para todo $1 \leq i \leq n$, os $\gamma_{i}$ são caminhos distintos em $Q$ de comprimento maior ou igual a 2, e o $\left(\gamma_{i}\right)=o\left(\gamma_{j}\right), t\left(\gamma_{i}\right)=t\left(\gamma_{j}\right)$ para todo $1 \leq i, j \leq n$.

Se $n=1$, a relação é dita uma relação zero ou relação monomial. Se $\rho$ é uma diferença de caminhos, então $\rho$ é dita uma relação de comutatividade.

Se $\left\{\rho_{j}\right\}_{j \in J}$ é um conjunto de relações de um carcás $Q$ tal que o ideal bilateral gerado por eles $\left\langle\rho_{j} \mid j \in J\right\rangle$ é admissível, dizemos que o carcás $Q$ é limitado pelas relações $\rho_{j}=0$ para todo $j \in J$.

Exemplo 4. No Exemplo 3 (4), as relações $\beta \eta$ e $\eta \delta \gamma$ são relações zero, e $\alpha \beta-\epsilon \zeta$ é uma relação de comutatividade. Dizemos que $Q$ está limitado pelas relações $\beta \eta=0, \eta \delta \gamma=0$ e $\alpha \beta=\epsilon \zeta$.

Lema 2. Seja $Q$ um carcás finito. Todo ideal admissível I é finitamente gerado. Aliás, existe um conjunto finito de relações $\left\{\rho_{1}, \ldots, \rho_{m}\right\}$ tal que $I=\left\langle\rho_{1}, \ldots, \rho_{m}\right\rangle$.

\subsection{Teorema de Gabriel}

Daqui em diante vamos assumir o corpo $k$ algebricamente fechado. Seja $A$ uma álgebra de dimensão finita sobre um corpo algebricamente fechado $k$. Pelo enunciado anteriormente podemos assumir $A$ básica e indecomponível. Vamos ver que neste caso $A$ é isomorfa a um quociente de uma álgebra de caminhos $k Q / I$, com $Q$ um carcás conexo finito e $I$ um ideal admissível de $k Q$, o que é conhecido como o Teorema de Gabriel.

Definição 16. Seja A uma k-álgebra básica e indecomponível de dimensão finita e seja $\left\{e_{1}, \ldots, e_{n}\right\}$ um conjunto completo de idempotentes primitivos ortogonais de $A$. O carcás $Q_{A}$ associado a $A$ é definido por:

- Os vértices de $Q_{A}$ são $1,2, \ldots, n$ em correspondência bijetiva com os idempotentes $e_{1}, e_{2}, \ldots, e_{n}$.

- Dados dois vértices $i, j$ em $Q_{A}$, o número de flechas de $i$ até $j$ está dado pela dimensão do $k$-espaço vetorial

$$
e_{i}\left(\operatorname{rad} A / \operatorname{rad}^{2} A\right) e_{j}
$$

Nota 1. - Como A é de dimensão finita o carcás obtido $Q_{A}$ é finito. 
- $Q_{A}$ não depende da escolha do conjunto de idempotentes primitivos ortogonais em A.

- $Q_{A}$ é conexo.

Exemplo 5. (1) Seja $A=k[x] /\left\langle x^{n}\right\rangle$, com $n \geq 1$, então $Q_{A}$ tem um único vértice pois o único idempotente não nulo de $A$ é 1 . Como $\langle\bar{x}\rangle^{n}=0$ e $A /\langle\bar{x}\rangle \cong k$ temos que $\operatorname{rad}(A)=\langle\bar{x}\rangle$, onde $\bar{x}=x+\left\langle x^{n}\right\rangle$ (Proposição 5). Logo, $\operatorname{rad}^{2}(A)=\left\langle\bar{x}^{2}\right\rangle$ e $\operatorname{dim}_{k}\left(\operatorname{rad}(A) / \operatorname{rad}^{2}(A)\right)=1$ : uma base de $\operatorname{rad}(A) / \operatorname{rad}^{2}(A)$ está dada pela classe de $\bar{x}$ no quociente. Pelo anterior o carcás associado a $A$ é :

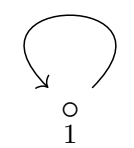

(2) Seja

$$
A=\left[\begin{array}{lll}
k & 0 & 0 \\
k & k & 0 \\
k & 0 & k
\end{array}\right]
$$

a álgebra de matrizes triangulares inferiores $\left[\lambda_{i j}\right] \in M_{3}(k)$, com $\lambda_{32}=0$ e $\lambda_{i j}=0$ para $i<j$. Um sistema completo de idempotentes primitivos ortogonais está dado por

$$
e_{1}=\left[\begin{array}{lll}
1 & 0 & 0 \\
0 & 0 & 0 \\
0 & 0 & 0
\end{array}\right], e_{2}=\left[\begin{array}{lll}
0 & 0 & 0 \\
0 & 1 & 0 \\
0 & 0 & 0
\end{array}\right], e_{3}=\left[\begin{array}{lll}
0 & 0 & 0 \\
0 & 0 & 0 \\
0 & 0 & 1
\end{array}\right] .
$$

É possivel ver que

$$
\operatorname{rad} A=\left[\begin{array}{lll}
0 & 0 & 0 \\
k & 0 & 0 \\
k & 0 & 0
\end{array}\right]
$$

$\operatorname{rad}^{2} A=0$, e e $e_{2}(\operatorname{rad} A) e_{1}$ e $e_{3}(\operatorname{rad} A) e_{1}$ têm dimensão 1 . As dimensões dos outros espaços tem que ser 0 pois $\operatorname{dim}_{k}(\operatorname{rad} A)=2$. Portanto $Q_{A}$ é o carcás

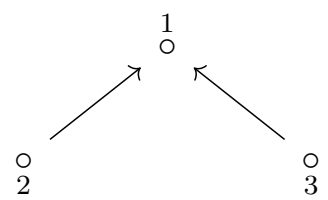

Podemos generalizar este exemplo tomando A como a álgebra de matrizes triangulares $n \times n$

$$
A=\left[\begin{array}{ccccc}
k & 0 & 0 & \ldots & 0 \\
k & k & 0 & \ldots & 0 \\
k & 0 & k & \ldots & 0 \\
\vdots & \vdots & & \ddots & \vdots \\
k & 0 & 0 & \ldots & k
\end{array}\right]
$$

com carcás associado 


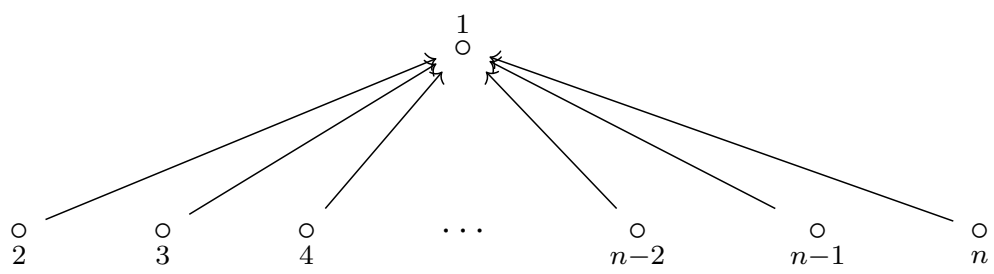

(3) Se $A=k Q / I$ é o quociente de uma álgebra de caminhos por um ideal admissivel, então $Q_{A}$ coincide com $Q$. Sabemos que o conjunto dos vértices $\left\{v_{1}, v_{2}, \ldots, v_{n}\right\}$ formam um conjunto completo de idempotentes primitivos ortogonais de $A$. E não é difícil verificar que $v_{i}\left(\operatorname{rad} A / \mathrm{rad}^{2} A\right) v_{j}$ tem dimensão o número de flechas em $Q$ de $v_{i}$ até $v_{j}$.

Agora vamos construir um homomorfismo sobrejetor $\phi: k Q_{A} \rightarrow A$ tal que $\operatorname{Ker} \phi$ é um ideal admissível e $A \cong k Q_{A} / \operatorname{Ker} \phi$.

Para toda flecha $\alpha: i \rightarrow j \in\left(Q_{A}\right)_{1}$, escolha $x_{\alpha} \in \operatorname{rad}(A)$ tal que $\left\{x_{\alpha}+\operatorname{rad}^{2}(A) \mid \alpha: i \rightarrow j\right\}$ é uma base de $e_{i}\left(\operatorname{rad} A / \operatorname{rad}^{2} A\right) e_{j}$.

Definimos $\phi$ sobre a base de $k Q_{A}$ como segue:

- $\phi\left(\tau_{i}\right):=e_{i}$ para todo caminho trivial $\tau_{i}$,

- $\phi(\alpha):=x_{\alpha}$ para toda $\alpha \in\left(Q_{A}\right)_{1}$,

- $\phi\left(\alpha_{1} \cdots \alpha_{n}\right):=x_{\alpha_{1}} \cdots x_{\alpha_{n}}$ para todo caminho de comprimento $n>1$.

Pela propriedade universal de álgebras de caminhos, que não demonstraremos aqui, existe um único homomorfismo de $k$-álgebras estendendo $\phi$. É possível mostrar que $\phi$ é um homomorfismo de $k$-álgebras sobrejetor cujo núcleo é um ideal admissível de $k Q_{A}$. Note que o ideal admissível $I=\operatorname{ker} \phi$ depende da escolha dos $x_{\alpha}$, assim podemos ter representações diferentes de $A$ tal que $k Q_{A} / I \cong k Q_{A} / I^{\prime}$.

Usando o Teorema Fundamental do Homomorfismo obtemos o seguinte resultado.

Teorema 3. Seja $k$ um corpo algebricamente fechado e $A$ uma k-álgebra de dimensão finita básica e indecomponivel. Existe um ideal admissivel $I$ de $k Q_{A}$ tal que $A \cong k Q_{A} / I$.

O Teorema anterior implica que é equivalente estudar álgebras indecomponíveis básicas de dimensão finita ao estudar quocientes de álgebras de caminhos por ideais admissíveis. O Teorema 3 aparece generalizado no livro [ARS97].

Exemplo 6. (1) Seja $A=k[x] /\left\langle x^{n}\right\rangle$ com $n \geq 1$, como no Exemplo 5. Definimos o homomorfismo $\phi: k Q_{A} \rightarrow A$ por $\phi\left(\tau_{1}\right)=1$ e $\phi(\alpha)=\bar{x}$. Definido $\phi$ deste jeito, é sobrejetor e com núcleo $\left\langle\alpha^{n}\right\rangle$.

(2) Seja $A$ a subálgebra de matrizes $3 \times 3$ do Exemplo 5 (2) e $\phi: k Q_{A} \rightarrow A$ definida por:

$$
\begin{gathered}
\phi\left(\tau_{1}\right)=\left[\begin{array}{lll}
1 & 0 & 0 \\
0 & 0 & 0 \\
0 & 0 & 0
\end{array}\right], \phi\left(\tau_{2}\right)=\left[\begin{array}{lll}
0 & 0 & 0 \\
0 & 1 & 0 \\
0 & 0 & 0
\end{array}\right], \phi\left(\tau_{3}\right)=\left[\begin{array}{lll}
0 & 0 & 0 \\
0 & 0 & 0 \\
0 & 0 & 1
\end{array}\right] . \\
\phi(\alpha)=\left[\begin{array}{lll}
0 & 0 & 0 \\
1 & 0 & 0 \\
0 & 0 & 0
\end{array}\right], \phi(\beta)=\left[\begin{array}{lll}
0 & 0 & 0 \\
0 & 0 & 0 \\
1 & 0 & 0
\end{array}\right]
\end{gathered}
$$

$\phi$ é um isomorfismo, $\operatorname{logo} A \cong k Q_{A}$. 


\section{Capítulo 2}

\section{Cohomologia de Hochschild}

Seja $k$ um anel comutativo com unidade e $A$ uma $k$-álgebra associativa com identidade. Neste capítulo apresentamos a definição do $i$-ésimo grupo de cohomologia de $\operatorname{Hochschild~} H H^{i}(A, M)$ de $A$ com coeficientes no $A$-bimódulo $M$. Apresentamos também uma resolução de $A$ sobre a sua álgebra envolvente $A^{e}:=A^{o p} \otimes A$, o que é equivalente a uma resolução de $A$ como $A$-bimódulo. Aplicando o funtor $\operatorname{Hom}_{A^{e}}(., M)$ a esta resolução obtemos um complexo cujas entradas e homomorfismos coincidem com os inicias para definir o $i$-ésimo grupo de cohomologia de Hochschild. No caso em que $k$ é um corpo esta resolução de bimódulos resulta ser uma resolução projetiva, permitindo assim definir $H H^{i}(A, M):=\operatorname{Ext}_{A e}^{i}(A, M)$. Na segunda seção do capítulo apresentamos a interpretação dos primeiros grupos de cohomologia e algumas consequências desses primeiros grupos se anularem em casos particulares de álgebras. Na terceira seção definimos duas resoluções de bimódulos que servem para calcular os grupos de cohomologia de álgebras hereditárias e álgebras de radical quadrado zero no caso de dimensão finita.

\subsection{Definição de cohomologia de Hochschild}

No Capítulo 1 definimos o conceito de álgebra sobre um corpo $k$, esse conceito continua válido se consideramos $k$ um anel comutativo e $A$ um anel que tem estrutura de $k$-módulo que satisfaz a condição adicional descrita na Definição 1. Vamos definir o $i$-ésimo grupo de cohomologia de Hochschild considerando álgebras sobre anéis comutativos e posteriormente vamos comprovar a necessidade de $k$ ser um corpo para calcular os grupos de cohomologia mediante resoluções projetivas.

No que resta desta dissertação notaremos o produto tensorial de $A n$ vezes sobre $k$ por $A^{\otimes n}:=A \otimes_{k} \cdots \otimes_{k} A$.

Definição 17. Seja $k$ um anel comutativo com unidade e seja A uma k-álgebra associativa com identidade. Dado um A-bimódulo $M$ definimos o Complexo de Hochschild como segue:

$$
\begin{aligned}
0 \longrightarrow M \stackrel{d^{0}}{\longrightarrow} & \operatorname{Hom}_{k}(A, M) \stackrel{d^{1}}{\longrightarrow} \operatorname{Hom}_{k}\left(A^{\otimes 2}, M\right) \longrightarrow \cdots \\
\cdots & \longrightarrow \operatorname{Hom}_{k}\left(A^{\otimes n}, M\right) \stackrel{d^{n}}{\longrightarrow} \operatorname{Hom}_{k}\left(A^{\otimes(n+1)}, M\right) \longrightarrow \ldots
\end{aligned}
$$

onde $\left(d^{0} m\right)(a)=$ am - ma para todo $m \in M, a \in A$ e $d^{n}$, com $n \geq 1$, está definido por

$$
\begin{aligned}
\left(d^{n} f\right)\left(a_{0} \otimes \cdots \otimes a_{n}\right)= & a_{0} f\left(a_{1} \otimes \cdots \otimes a_{n}\right) \\
& +\sum_{i=0}^{n-1}(-1)^{i+1} f\left(a_{0} \otimes \cdots \otimes a_{i} a_{i+1} \otimes \cdots \otimes a_{n}\right) \\
& +(-1)^{n+1} f\left(a_{0} \otimes \cdots \otimes a_{n-1}\right) a_{n},
\end{aligned}
$$

para $f \in \operatorname{Hom}_{k}\left(A^{\otimes n}, M\right)$ e $a_{0}, \ldots, a_{n} \in A$. 
Por exemplo, dado $f \in \operatorname{Hom}_{k}(A, M), g \in \operatorname{Hom}_{k}\left(A^{\otimes 2}, M\right)$ e $a, b, c \in A$ temos que

$$
\begin{aligned}
\left(d^{1} f\right)(a \otimes b) & =a f(b)-f(a b)+f(a) b \\
\left(d^{2} g\right)(a \otimes b \otimes c) & =a g(b \otimes c)-g(a b \otimes c)+g(a \otimes b c)-g(a \otimes b) c
\end{aligned}
$$

A demonstração direta de que a sequência dada na definição anterior é de fato um complexo é apresentada em [Hoc45]. Logo, podemos definir o i-ésimo grupo de cohomologia de $A$ com coeficientes no bimódulo $M$, dado por $H H^{i}(A, M)=\operatorname{ker}\left(d^{i}\right) / \operatorname{Im}\left(d^{i-1}\right)$ para $i \geq 1$. No caso $i=0$ temos $H H^{0}(A, M)=\operatorname{Ker}\left(d^{0}\right)$.

Um caso particular de interesse é quando tomamos $M=A$. Neste caso notamos $H H^{i}(A, A)$ simplesmente por $H H^{i}(A)$.

A cohomologia de Hochschild fornece informações de uma álgebra dada, como por exemplo se ela é separável. Mais adiante definiremos o que significa que uma álgebra seja separável. Logo mostraremos uma condição suficiente de separabilidade envolvendo o primeiro grupo de cohomologia e para isto faremos uso do seguinte Teorema.

Teorema 4. [Hoc45] Se $n \geq 2, H H^{n}(A, M) \cong H H^{n-1}\left(A, H_{o} m_{k}(A, M)\right)$.

A seguir apresentamos a definição de álgebra envolvente, a qual nos fornece outra maneira de calcular os grupos de cohomologia de Hochschild no caso em que a álgebra $A$ é um módulo projetivo sobre $k$. O que acontece sempre quando $k$ é um corpo, que é o que assumimos em toda esta dissertação.

Definição 18. Sejam $k$ um anel comutativo com unidade, $A$ uma k-álgebra associativa com identidade e considere o $k$-módulo $A^{e}:=A^{o p} \otimes_{k} A$ junto com o produto

$$
\left(a_{1} \otimes b_{1}\right) \cdot\left(a_{2} \otimes b_{2}\right)=\left(a_{2} a_{1} \otimes b_{1} b_{2}\right),
$$

para todo $a_{1}, a_{2}, b_{1}, b_{2} \in A$. Chamamos $A^{e}$ de álgebra envolvente de $A$.

Dado um $A$-bimódulo $M$, podemos definir uma estrutura sobre $M$ de $A^{e}$-módulo à esquerda $($ resp. à direita $)$ mediante o produto $(a \otimes b) \cdot m=a m b($ resp. $m \cdot(a \otimes b)=b m a)$. Esta estrutura define um isomorfismo entre a categoria de $A$-bimódulos e a categoria de $A^{e}$-módulos à esquerda (resp. $A^{e}$-módulos à direita).

O produto tensorial $A^{\otimes n}$ é um $A^{e}$-módulo à esquerda (equiv. $A$-bimódulo) com o produto dado por

$$
(a \otimes b) \cdot\left(a_{1} \otimes a_{2} \otimes \cdots \otimes a_{n}\right)=a a_{1} \otimes a_{2} \otimes \cdots \otimes a_{n-1} \otimes a_{n} b,
$$

para todo $a, b, a_{1}, \ldots, a_{n} \in A$. Em particular, $A$ é um $A^{e}$-módulo a esquerda (equiv. $A$-bimódulo) com o produto dado por $(a \otimes b) \cdot c=a c b$ para todo $a, b, c \in A$.

Lema 3. Considere a seguinte sequência de A-bimódulos

$$
\cdots \stackrel{f_{3}}{\longrightarrow} A^{\otimes 4} \stackrel{f_{2}}{\longrightarrow} A^{\otimes 3} \stackrel{f_{1}}{\longrightarrow} A \otimes A \stackrel{f_{0}}{\longrightarrow} A \longrightarrow 0,
$$

$\operatorname{com} f_{n}: A^{\otimes(n+2)} \rightarrow A^{\otimes(n+1)}$ dada por

$$
f_{n}\left(a_{0} \otimes a_{1} \otimes \cdots \otimes a_{n+1}\right)=\sum_{i=0}^{n}(-1)^{i} a_{0} \otimes a_{1} \otimes \cdots \otimes a_{i} a_{i+1} \otimes \cdots \otimes a_{n+1},
$$

para todo $n \geq 0$ e $a_{0}, \ldots, a_{n+1} \in A$. 
A sequência anterior é uma resolução de $A$ sobre $A^{e}$ e a chamamos de resolução de Hochschild.

Demonstração. Seja $s_{n}: A^{\otimes n} \rightarrow A^{\otimes(n+1)}$ dada por $s_{n}\left(a_{0} \otimes \cdots \otimes a_{n-1}\right)=1 \otimes a_{0} \otimes \cdots \otimes a_{n-1}$, para todo $a_{0}, \ldots, a_{n-1} \in A$. A aplicação $s_{1}$ satisfaz $f_{0} s_{1}=i d_{A}$, o que implica que $f_{0}$ é um epimorfismo. Além disso, pode-se mostrar por indução que $f_{n} s_{n+1}+s_{n} f_{n-1}=i d_{A^{\otimes(n+1)}}$ para todo $n \geq 1$, portanto $\operatorname{ker}\left(f_{n-1}\right) \subseteq \operatorname{Im}\left(f_{n}\right)$ para todo $n \geq 1$.

Vamos mostrar por indução que $f_{n} f_{n+1}=0$ para todo $n \geq 0$. Para o caso $n=0$ usamos o fato da álgebra ser associativa. Sejam $a, b, c \in A$, então

$$
f_{0} f_{1}(a \otimes b \otimes c)=f_{0}(a b \otimes c-a \otimes b c)=(a b) c-a(b c)=0 .
$$

Agora, vamos supor que $f_{n-1} f_{n}=0, \operatorname{logo}$

$$
f_{n} f_{n+1} s_{n+2}=f_{n}\left(i d_{A^{\otimes(n+2)}}-s_{n+1} f_{n}\right)=\left(i d_{A^{\otimes(n+2)}}-f_{n} s_{n+1}\right) f_{n}=s_{n} f_{n-1} f_{n}=0
$$

Como $\operatorname{Im}\left(s_{n+2}\right)$ gera $A^{\otimes(n+3)}$ como $A$-módulo e $f_{n}$ é um homomorfismo de $A$-módulos para todo $n \geq 0$, então $f_{n} f_{n+1}=0$.

Definimos o complexo bar como sendo o complexo truncado obtido de (2.1)

$$
\cdots \stackrel{f_{3}}{\longrightarrow} A^{\otimes 4} \stackrel{f_{2}}{\longrightarrow} A^{\otimes 3} \stackrel{f_{1}}{\longrightarrow} A \otimes A \longrightarrow 0 .
$$

Seja $M$ um $A$-bimódulo. Aplicando o funtor $\operatorname{Hom}(., M)$ a $(2.2)$ e usando o isomorfismo de $k$-módulos

$$
\operatorname{Hom}_{A^{e}}\left(A^{\otimes(n+2)}, M\right) \cong \operatorname{Hom}_{k}\left(A^{\otimes n}, M\right),
$$

dado por $\psi(f)=\tilde{f}$, com $\tilde{f}\left(a_{0} \otimes \cdots \otimes a_{n-1}\right)=f\left(1 \otimes a_{0} \otimes \cdots \otimes a_{n-1} \otimes 1\right)$, para todo $f \in$ $\operatorname{Hom}_{A^{e}}\left(A^{\otimes(n+2)}, M\right)$ e todo $a_{0}, \ldots, a_{n-1} \in A$, ( o isomorfismo inverso está dado por $\varphi(f)=f^{\prime}$, $\left.\operatorname{com} f^{\prime}\left(a_{0} \otimes \cdots \otimes a_{n+1}\right)=a_{0} f\left(a_{1} \otimes \cdots \otimes a_{n}\right) a_{n+1}\right)$ obtemos o seguinte isomorfismo de complexos

$$
\begin{aligned}
& \cdots \rightarrow \operatorname{Hom}_{A^{e}}\left(A^{\otimes(n+2)}, M\right) \stackrel{f_{n+1}^{*}}{\longrightarrow} \operatorname{Hom}_{A^{e}}\left(A^{\otimes(n+3)}, M\right) \rightarrow \cdots \\
& \cdots \rightarrow \operatorname{Hom}_{k}\left(A^{\otimes n}, M\right) \stackrel{\delta^{n}}{\longrightarrow} \operatorname{Hom}_{k}\left(A^{\otimes(n+1)}, M\right) \rightarrow \cdots
\end{aligned}
$$

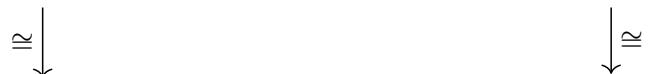

onde $f_{n+1}^{*}(g)=g f_{n+1}$ para todo $g \in \operatorname{Hom}_{A^{e}}\left(A^{\otimes(n+2)}, M\right)$. Os homomorfismos $\delta^{n}$ se definem de modo que os quadrados comutem, logo

$$
\begin{aligned}
\left(\delta^{n} f\right)\left(a_{0} \otimes \cdots \otimes a_{n}\right) & =\left(\psi f_{n+1}^{*} \varphi\right)(f)\left(a_{0} \otimes \cdots \otimes a_{n}\right) \\
& =\psi\left(\varphi(f) f_{n+1}\right)\left(a_{0} \otimes \cdots \otimes a_{n}\right) \\
& =\varphi(f) f_{n+1}\left(1 \otimes a_{0} \otimes \cdots \otimes a_{n} \otimes 1\right)
\end{aligned}
$$

Usando a definição de $f_{n+1}$ obtemos 


$$
\begin{aligned}
\left(\delta^{n} f\right)\left(a_{0} \otimes \cdots \otimes a_{n}\right)=\varphi( & f)\left(a_{0} \otimes a_{1} \otimes \cdots \otimes a_{n} \otimes 1+\right. \\
+ & \sum_{i=0}^{n-1}(-1)^{i+1} 1 \otimes a_{0} \otimes a_{1} \otimes \cdots \otimes a_{i} a_{i+1} \otimes \cdots \otimes a_{n} \otimes 1+ \\
& \left.+(-1)^{n+1} 1 \otimes a_{0} \otimes \cdots \otimes a_{n-1} \otimes a_{n}\right) \\
= & a_{0} f\left(a_{1} \otimes \cdots \otimes a_{n}\right) \\
& +\sum_{i=0}^{n-1}(-1)^{i+1} f\left(a_{0} \otimes \cdots \otimes a_{i} a_{i+1} \otimes \cdots \otimes a_{n}\right) \\
& +(-1)^{n+1} f\left(a_{0} \otimes \cdots \otimes a_{n-1}\right) a_{n}
\end{aligned}
$$

Note que o homomorfismo $\delta^{n}$ coincide com o homomorfismo $d^{n}$ dado na definição de complexo de Hochschild.

No caso em que $A$ é projetivo sobre $k$ (em particular, se $k$ é um corpo ) o complexo (2.2) é uma resolução de $A^{e}$-módulos livres à esquerda, chamada de resolução bar [Red01]. Então concluímos que a cohomologia de Hochschild de $A$ com coeficientes no bimódulo $M$ é

$$
H H^{n}(A, M)=\operatorname{Ext}_{A^{e}}^{n}(A, M) .
$$

Pelo anterior, o $i$-ésimo grupo de cohomologia de Hochschild não depende da resolução projetiva de $A$ sobre $A^{e}$. Para algumas álgebras especificas existem resoluções projetivas diferentes da resolução bar que facilitam os cálculos.

Nota 2. Aplicando o funtor $M \otimes_{A^{e}}$ à̀ resolução (2.1) obtemos o conceito dual de homologia de Hochschild definido por

$$
H H_{i}(A, M)=\operatorname{Tor}_{i}^{A^{e}}(M, A) .
$$

Temos dois isomorfismos importantes que relacionam a cohomologia e a homologia de Hochschild [CE16] :

$$
\begin{aligned}
& H H^{n}\left(A, H_{\text {om }}(M, k)\right) \simeq \operatorname{Hom}_{k}\left(H H_{n}(A, M), k\right) \\
& H H_{n}\left(A, H_{k}(M, k)\right) \simeq \operatorname{Hom}_{k}\left(H H^{n}(A, M), k\right)
\end{aligned}
$$

Definição 19. Sejam $f \in \operatorname{Hom}_{k}\left(A^{\otimes m}, A\right)$ e $g \in \operatorname{Hom}_{k}\left(A^{\otimes n}, A\right)$, definimos o produto cup $f \smile g \in \operatorname{Hom}_{k}\left(A^{\otimes(m+n)}, A\right)$ por

$$
(f \smile g)\left(a_{1} \otimes \cdots \otimes a_{m+n}\right)=f\left(a_{1} \otimes \cdots \otimes a_{m}\right) g\left(a_{m+1} \otimes \cdots \otimes a_{m+n}\right)
$$

para todo $a_{1}, \ldots, a_{m+n} \in A$.

Esse produto definido no complexo satisfaz a regra de Leibnitz e por isso induz um produto associativo na soma direta das cohomologias

$$
H H^{*}(A)=\bigoplus_{i \geq 0} H H^{i}(A) .
$$

Chamamos esta álgebra associativa de álgebra de cohomologia de $A$ [Ger63].

Nota 3. A cohomologia de Hochschild é invariante por equivalências derivadas, em particular por equivalência de Morita. Isto é, dadas duas k-álgebras $A$ e $B$ tais que $D^{b}(A)$ é triangularmente equivalente a $D^{b}(B)$, então $H H^{i}(A) \cong H H^{i}(B)$, para todo $i \geq 0$. [Wei95] 
A seguir, apresentamos um exemplo que mostra que existem resoluções mais simples que a resolução bar com o fim de calcular a cohomologia de Hochschild. Este exemplo é um caso particular do feito em [Gro91].

Exemplo 7. Seja $A=k[x] /\left\langle x^{n}\right\rangle$, onde $k$ é um anel comutativo com unidade e $n \geq 2$. Vamos calcular a cohomologia de Hochschild de A com coeficientes no bimódulo A. Como A é livre sobre $k$, então $A$ é $k$-projetivo e podemos considerar qualquer resolução projetiva de $A$ sobre $A^{e}$. Consideremos o complexo

$$
\cdots \longrightarrow A^{e} \stackrel{d_{n}}{\longrightarrow} A^{e} \longrightarrow \cdots \longrightarrow A^{e} \stackrel{d_{1}}{\longrightarrow} A^{e} \stackrel{\mu}{\longrightarrow} A \longrightarrow 0
$$

onde

$$
\begin{aligned}
d_{2 i}(a \otimes b) & =(a \otimes b) \cdot \sum_{j=0}^{n-1} x^{j} \otimes x^{n-1-j}, \\
d_{2 i-1}(a \otimes b) & =(a \otimes b) \cdot(1 \otimes x-x \otimes 1), \\
\mu(a \otimes b) & =a \cdot b,
\end{aligned}
$$

para todo $a, b \in A$ e todo $i \geq 1$.

Um cálculo direto mostra que $\operatorname{Im}\left(d_{1}\right) \subseteq \operatorname{Ker}(\mu)$, e $\operatorname{Im}\left(d_{2 i}\right) \subseteq \operatorname{Ker}\left(d_{2 i-1}\right)$ para todo $i \geq 1$. De fato,

$$
\begin{aligned}
& \mu d_{1}(a \otimes b)=\mu((a \otimes b) \cdot(1 \otimes x-x \otimes 1))=\mu(a \otimes b x-a x \otimes b)=0 \\
& d_{2 i-1} d_{2 i}(a \otimes b)=d_{2 i-1}\left((a \otimes b) \cdot \sum_{j=0}^{n-1} x^{j} \otimes x^{n-1-j}\right) \\
&=d_{2 i-1}\left(\sum_{j=0}^{n-1} a x^{j} \otimes b x^{n-1-j}\right) \\
&=\left(\sum_{j=0}^{n-1} a x^{j} \otimes b x^{n-1-j}\right) \cdot(1 \otimes x-x \otimes 1) \\
&=\left(\sum_{j=0}^{n-1} a x^{j} \otimes b x^{n-j}\right)-\left(\sum_{j=0}^{n-1} a x^{j+1} \otimes b x^{n-1-j}\right) \\
&=a \otimes b x^{n}-a x^{n} \otimes b \\
&=0
\end{aligned}
$$

Analogamente $\operatorname{Im}\left(d_{2 i+1}\right) \subseteq \operatorname{Ker}\left(d_{2 i}\right)$.

Sejam $s_{0}: A \rightarrow A^{2}, s_{1}: A^{2} \rightarrow A^{2}, s_{2}: A^{2} \rightarrow A^{2}$ as seguintes aplicações A-lineares

$$
s_{0}\left(x^{j}\right)=1 \otimes x^{j}, s_{1}\left(x^{j} \otimes 1\right)=-\sum_{l=1}^{j} x^{l-1} \otimes x^{j-l}, s_{2}\left(x^{j} \otimes 1\right)=\delta_{j, n-1} \otimes 1,
$$

onde $\delta_{j, n-1}$ e o delta de Kronecker. Estas aplicações satisfazem as seguintes igualdades

$$
\mu s_{0}=i d, \quad s_{0} \mu+d_{1} s_{1}=i d, \quad s_{2} d_{2 i}+d_{2 i+1} s_{1}=i d,
$$

o que implica que (2.3) é uma resolução livre de $A$ sobre $A^{e}$. 
Agora, aplicando o funtor $\operatorname{Hom}_{A^{e}}(\cdot, A)$ a (2.3) e usando o isomorfismo $\operatorname{Hom}_{A^{e}}(A \otimes A, A) \cong A$, calcular os grupos de cohomologia de Hochschild se reduz a calcular os grupos de cohomologia do seguinte complexo

$$
0 \longrightarrow A \stackrel{b_{1}}{\longrightarrow} A \stackrel{b_{2}}{\longrightarrow} \cdots \longrightarrow A \stackrel{b_{n}}{\longrightarrow} A \stackrel{b_{n+1}}{\longrightarrow} \cdots,
$$

onde $b_{2 i-1}=0$ e $b_{2 i}(a)=n x^{n-1}$ a para todo $i \geq 1$ e todo $a \in A$.

Se $n=0$ em $k$ então $H H^{i}(A)=A$ para todo $i \geq 0$. Se $n \neq 0$ em $k$, então $H H^{0}(A)=A, H H^{2 i}(A)=A / n x^{n-1} A$ e $H H^{2 i-1}(A)=A n n\left(n x^{n-1}\right)=(x)$ para todo $i \geq 1$.

\subsection{Interpretação dos primeiros grupos de cohomologia}

Dada um álgebra $A$ os grupos de cohomologia $H H^{i}(A)$ com $i=0,1,2$ têm interpretações concretas as quais apresentamos nesta seção usando os morfismo e módulos da Definição 17. Também enunciamos e mostramos alguns resultados no caso em que estes grupos são nulos.

\subsubsection{0-ésimo grupo de cohomologia}

Seja $A$ uma $k$-álgebra e $M$ um $A$-bimódulo. Tendo em conta a Definição 17, a 0-ésima cohomologia de $A$ com coeficientes em $M$ está dada por

$$
H H^{0}(A, M)=\operatorname{Ker}\left(d^{0}\right)=\{m \in M \mid a m=m a, \forall a \in A\} .
$$

No caso particular em que $M=A$, temos $H H^{0}(A)=Z(A)$, o centro da álgebra $A$.

\subsubsection{Primeiro grupo de cohomologia}

Definição 20. Seja A uma álgebra e $M$ um A-bimódulo. O espaço de $k$-derivações de $A$ em $M$ está dado por

$$
\operatorname{Der}(A, M)=\left\{f \in H_{o m}(A, M) \mid f(a b)=a f(b)+f(a) b, \forall a, b \in A\right\} .
$$

O subespaço de $\operatorname{Der}(A, M)$ de derivações internas de $A$ em $M$ está dado por

$$
\operatorname{Der}^{0}(A, M)=\left\{f_{m} \in \operatorname{Hom}_{k}(A, M), m \in M \mid f_{m}(a)=a m-m a, \forall a \in A\right\} .
$$

Note que $\operatorname{Ker}\left(d^{1}\right)=\operatorname{Der}(A, M)$ e $\operatorname{Im}\left(d^{0}\right)=\operatorname{Der}^{0}(A, M)$. Portanto, podemos expressar o primeiro grupo de cohomologia de Hochschild em termos dos espaços de derivações:

$$
H H^{1}(A, M)=\operatorname{Der}(A, M) / \operatorname{Der}^{0}(A, M) .
$$

No caso particular em que $M=A$ notamos $\operatorname{Der}(A, A)$ e $\operatorname{Der}^{0}(A, A)$ por $\operatorname{Der}(A)$ e $\operatorname{Der}^{0}(A)$ respectivamente. $\operatorname{Der}(A)$ é chamado espaço de derivações de $A$ e $\operatorname{Der}^{0}(A)$ o espaço de derivações internas de $A$.

$\mathrm{Na}$ demonstração do seguinte Teorema completamos os detalhes da demonstração encontrada em [Hap89] do Lema 3.1.

Teorema 5. Seja A uma k-álgebra de dimensão finita, onde $k$ é um corpo de característica zero e seja $\left\{e_{1}, e_{2}, \ldots, e_{n}\right\}$ um sistema completo de idempotentes ortogonais primitivos de $A$. Se $\delta \in$ $\operatorname{Der}(A)$, existe $\delta^{\prime} \in \operatorname{Der}^{0}(A)$ tal que $\delta\left(e_{i}\right)=\delta^{\prime}\left(e_{i}\right)$ para $1 \leq i \leq n$.

Demonstração. Como $\delta\left(e_{i}\right)=\delta\left(e_{i}^{2}\right)=e_{i} \delta\left(e_{i}\right)+\delta\left(e_{i}\right) e_{i}$, então $e_{i} \delta\left(e_{i}\right) e_{i}=0$. Logo, $e_{i} \delta\left(e_{i}\right) \in e_{i} A(1-$ $\left.e_{i}\right)$ e $\delta\left(e_{i}\right) e_{i} \in\left(1-e_{i}\right) A e_{i}$. Além disso, para $i \neq j, 0=\delta\left(e_{i} e_{j}\right)=e_{i} \delta\left(e_{j}\right)+\delta\left(e_{i}\right) e_{j}$. 
Como $e_{i} \delta\left(e_{i}\right) \in e_{i} A\left(1-e_{i}\right)$, existe $a \in A$ tal que $e_{i} \delta\left(e_{i}\right)=e_{i} a\left(1-e_{i}\right)$. Usando a decomposição de Peirce ([DK12] pág 26.), podemos expressar $a$ da forma

$$
a=\sum_{k, j} e_{k} a_{k j} e_{j}
$$

então

$$
e_{i} \delta\left(e_{i}\right)=\sum_{j \neq i} e_{i} a_{i j} e_{j}
$$

Vamos notar por $\gamma_{i j}$ o termo $e_{i} a_{i j} e_{j}$ na decomposição de $e_{i} \delta\left(e_{i}\right)$ e fixamos $\gamma_{i i}=0$. Afirmamos $\delta\left(e_{i}\right) e_{i}=-\sum_{j=1}^{n} \gamma_{j i}$. Seja $i$ fixo tal que $1 \leq i \leq n$, e considere a soma

$$
\sum_{j=1, j \neq i}^{n} e_{j} \delta\left(e_{j}\right) e_{i}
$$

Por um lado temos que

$$
\sum_{j=1, j \neq i}^{n} e_{j} \delta\left(e_{j}\right) e_{i}=\sum_{j=1, j \neq i}^{n} \sum_{k \neq j} e_{j} a_{j k} e_{k} e_{i}=\sum_{j=1}^{n} e_{j} a_{j i} e_{i}=\sum_{j=1}^{n} \gamma_{j i},
$$

e por outro lado

$$
\sum_{j=1, j \neq i}^{n} e_{j} \delta\left(e_{j}\right) e_{i}=-\sum_{j=1, j \neq i}^{n} e_{j}\left(e_{j} \delta\left(e_{i}\right)\right)=\left(e_{i}-1\right) \delta\left(e_{i}\right)=-\delta\left(e_{i}\right) e_{i} .
$$

Portanto $\delta\left(e_{i}\right)=\sum_{i=1}^{n} \gamma_{i j}-\gamma_{j i}$. Seja $\gamma=\sum_{i, j} \gamma_{i j}$ e $\delta^{\prime}=\delta_{\gamma}$ a derivação interna associada a $\gamma$, logo $\delta_{\gamma}\left(e_{i}\right)=e_{i} \gamma-\gamma e_{i}=\sum_{i=1}^{n} \gamma_{i j}-\gamma_{j i}=\delta\left(e_{i}\right)$.

Definição 21. Dada uma álgebra $A$, definimos o subespaço de derivações normalizadas de $A$ como o conjunto $\left\{\delta \in \operatorname{Der}(A) \mid \delta\left(e_{i}\right)=0,1 \leq i \leq n\right\}$, o qual denotamos por $\operatorname{Der}^{n}(A)$.

Note que se $\delta \in \operatorname{Der}^{n}(A)$ e $a \in e_{i} A e_{j}$, então $\delta(a)=e_{i} \delta(a) e_{j} \in e_{i} A e_{j}$.

Lema 4. $H H^{1}(A) \cong \operatorname{Der}^{n}(A) / \operatorname{Der}^{n, 0}(A)$, onde $\operatorname{Der}^{n, 0}:=\operatorname{Der}^{n}(A) \cap \operatorname{Der}^{0}(A)$.

Demonstração. Sabemos que $H H^{1}(A)=\operatorname{Der}(A) / \operatorname{Der}^{0}(A)$. Defina o homomorfismo $\phi: \operatorname{Der}^{n}(A) \rightarrow$ $\operatorname{Der}(A) / \operatorname{Der}^{0}(A)$ dado por $\phi(\delta)=\bar{\delta}$. Seja $\bar{\delta} \in \operatorname{Der}(A) / \operatorname{Der}^{0}(A)$, pelo Teorema 5 existe $\delta^{\prime} \in \operatorname{Der}(A)$ tal que $\delta^{\prime}\left(e_{i}\right)=\delta\left(e_{i}\right)$ para todo $1 \leq i \leq n$. Defina $\delta^{\prime \prime}:=\delta-\delta^{\prime}$, então $\delta \in \operatorname{Der}^{n}(A)$ e $\phi\left(\delta^{\prime \prime}\right)=\bar{\delta}$. Claramente $\operatorname{Ker}(\phi)=\operatorname{Der}^{n, 0}$.

Se $A$ é comutativo a única derivação interna de $A$ é a aplicação nula, logo $H H^{1}(A)$ é simplesmente o espaço de derivações de $A$, que nesse caso é isomorfo ao espaço de derivações normalizadas de $A$.

Exemplo 8. Seja $Q$ um carcás finito e $k Q$ a sua álgebra de caminhos associada, com $k$ um corpo de característica zero. Seja I um ideal admissivel de $k Q$ homogêneo com relação ao grau, isto é, I é gerado por combinações lineares de caminhos que tem o mesmo comprimento e seja $A=k Q / I$. Vamos mostrar que se $H H^{1}(A)=0$, então $Q$ não tem ciclos. De fato:

Considere $\delta: k Q / I \rightarrow k Q / I$ dada por $\delta(\bar{w})=l(w) \bar{w}$ para todo caminho $w$ sobre $Q$, onde $l(w)$ é o comprimento de $w$. Note que $\delta$ está bem definida pelo fato de I ser homogêneo. Além disso, se $w_{1}$ e $w_{2}$ são dois caminhos tais que $\overline{w_{1} w_{2}} \neq 0$ então

$$
\delta\left(\overline{w_{1} w_{2}}\right)=l\left(w_{1} w_{2}\right) \overline{w_{1} w_{2}}=\left(l\left(w_{1}\right)+l\left(w_{2}\right)\right) \overline{w_{1} w_{2}}=l\left(w_{1}\right) \overline{w_{1} w_{2}}+l\left(w_{2}\right) \overline{w_{1} w_{2}}=\delta\left(\overline{w_{1}}\right) \overline{w_{2}}+\overline{w_{1}} \delta\left(\overline{w_{2}}\right)
$$


Portanto

$$
\delta\left(\overline{w_{1} w_{2}}\right)=\delta\left(\overline{w_{1}}\right) \overline{w_{2}}+\overline{w_{1}} \delta\left(\overline{w_{2}}\right) .
$$

Para o caso em que $\overline{w_{1} w_{2}}=0$ a igualdade (2.4) se satisfaz trivialmente. Logo $\delta$ é uma derivação sobre A. Como $\delta\left(\overline{\tau_{i}}\right)=l\left(\tau_{i}\right) \overline{\tau_{i}}=0$ para todo $i$ então $\delta$ é uma derivação normalizada.

$A$ hipótese $H H^{1}(A)=0$ implica que $\delta$ é uma derivação interna e portanto existe $\bar{a} \neq 0$ em $A$ tal que $\delta=\delta_{\bar{a}}$. Como $\delta \in \operatorname{Der}^{n}(A)$, então $0=\delta\left(\overline{\tau_{i}}\right)=\delta_{a}\left(\tau_{i}\right)=e_{i} a-a e_{i}$. Logo a é da forma

$$
a=\sum_{i=1}^{n} \mu_{i} e_{i}+y,
$$

onde $\mu_{i} \in k$ para $1 \leq i \leq n$ e $y \in \oplus_{i} e_{i}(\operatorname{rad} A) e_{i}$.

Seja $\alpha$ uma flecha em $Q$ e $\bar{\alpha}$ a sua classe em A. Temos que $\bar{\alpha}=\delta(\bar{\alpha})=\delta_{a}(\bar{\alpha})=\bar{\alpha} a-a \bar{\alpha}=$ $\mu_{t(\alpha)} \bar{\alpha}-\mu_{o(\alpha)} \bar{\alpha}+z$, com $z \in \operatorname{rad}^{2}(A)$. Logo, $\mu_{t(\alpha)}-\mu_{o(\alpha)}=1$ para toda $\alpha \in Q_{1}$. Suponha que existe um ciclo em $Q$

$$
1 \stackrel{\alpha_{1}}{\longrightarrow} 2 \stackrel{\alpha_{2}}{\longrightarrow} 3 \rightarrow \cdots \rightarrow n \stackrel{\alpha_{n}}{\longrightarrow} 1 \text {. }
$$

Pelo anterior devemos ter $\mu_{i+1}-\mu_{i}=1$ para $1 \leq i \leq n-1$ e $\mu_{1}-\mu_{n}=1$. Este sistema de equações não tem solução, pois char $(k)=0$, e obtemos uma contradição.

Definição 22. Sejam $k$ um corpo e A uma k-álgebra. Dizemos que A é separável se para toda extensão $L$ de $k, A_{L}=A \otimes L$ é semi-simples.

Teorema 6. [Hoc45] Uma álgebra $A$ é separável se e somente se $H H^{1}(A, M)=0$ para todo $A$ bimódulo $M$.

Lema 5. As seguintes afirmações são equivalentes:

(a) A é separável.

(b) $H H^{i}(A, M)=0$ para todo A-bimódulo $M$, e todo $i>0$.

(c) A é $A^{e}$-projetivo.

(d) Existe $a \otimes b \in A^{e}$ tal que $a b=1$ e $c(a \otimes b)=(a \otimes b) c$ para todo $c \in A$.

Demonstração. O Teorema (4) e o Teorema (6) mostram a equivalência $(a) \Leftrightarrow(b)$.

$(c) \Rightarrow(d)$ Suponha que $A$ é $A^{e}$-projetivo. Então o $A^{e}$-epimorfismo $\mu: A^{e} \rightarrow A$, onde $\mu(a \otimes b)=a b$ , cinde. Logo, existe um $A^{e}$-homomorfismo $\sigma: A \rightarrow A^{e}$ tal que $\mu \sigma=i d_{A}$. Seja $e=\sigma(1)$, então $\mu(e)=\mu(\sigma(1))=1$ e $a e=a \sigma(1)=\sigma(a)=\sigma(1) a=e a$.

$(d) \Rightarrow(c)$ Defina $\sigma: A \rightarrow A^{e}$ por $\sigma(a)=a e$. Então $\mu \sigma=i d_{A}, \mu$ é um epimorfismo que cinde e $A$ é $A^{e}$-projetivo.

A equivalência $(a) \Leftrightarrow(c)$ é mostrada em [DK12] para álgebras de dimensão finita.

Exemplo 9. Seja $A=M_{n}(k)$, o elemento

$$
e=\sum_{i=1}^{n} e_{i 1} \otimes e_{1 i}
$$

satisfaz as condições de $(d)$ no Lema anterior, logo, $H H^{i}(A, M)=0$ para todo $i>0$ e todo $M_{n}(k)$ bimódulo $M$. Observe que poderíamos obter este resultado pelo fato da álgebra $M_{n}(k)$ ser Morita equivalente a $k$ [ASS06][pág. 38].

\subsubsection{Segundo grupo de cohomologia}

Dada uma álgebra $A$ e um $A$-bimódulo $M$, existe uma correspondência entre as extensões de $A$ por $M$ e o segundo grupo de cohomologia $H H^{2}(A, M)$. A seguir, apresentamos uma demonstração desta correspondência a qual pode ser encontrada em [Red01]. 
Definição 23. Seja A uma k-álgebra .Uma extensão de $A$ é um epimorfismo de k-álgebras $\phi: B \rightarrow A$ que cinde. Dado um A-bimódulo $M$, uma extensão de $A$ por $M$ é uma sequência exata curta

$$
0 \rightarrow M \stackrel{i}{\rightarrow} B \stackrel{\phi}{\rightarrow} A \rightarrow 0
$$

com $i$ um monomorfismo de $k$-módulos tal que

$$
\begin{aligned}
& i(\phi(b) \cdot m)=b \cdot i(m) \\
& i(m \cdot \phi(b))=i(m) \cdot b
\end{aligned}
$$

para todo $b \in B$, e todo $m \in M$.

Dizemos que duas extensões de $A$ por $M$ são equivalentes se existe um isomorfismo $\phi: B \rightarrow B^{\prime}$ que faz o seguinte diagrama comutar

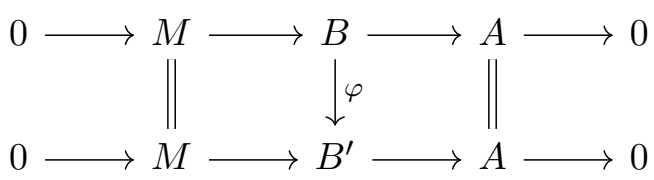

Dada uma extensão de $A$ por $M$

$$
0 \rightarrow M \stackrel{i}{\rightarrow} B \stackrel{\phi}{\rightarrow} A \rightarrow 0
$$

existe $\psi: A \rightarrow B k$-linear tal que $\phi \psi=i d_{A}$, logo temos o isomorfismo de $k$-módulos $B \cong A \oplus M$. Este isomorfismo é um isomorfismo de álgebras no caso em que $\psi$ é um morfismo de álgebras. Em geral $\psi$ não é um morfismo de álgebras, mas podemos medir "quão longe"está $\psi$ de ser um homomorfismo de álgebras por meio da aplicação $f: A \otimes A \rightarrow B$ definida por

$$
f(a \otimes b)=\psi(a b)-\psi(a) \psi(b)
$$

Note que $\operatorname{Im}(f) \subseteq \operatorname{Ker}(\phi)$ e como $\psi$ é $k$-linear temos $f: A \otimes A \rightarrow M$. Portanto, podemos definir o produto em $B \cong A \oplus M$ por

$$
(a, m) \cdot(b, n)=(a b, a n+m b+f(a \otimes b)) .
$$

Como $B$ fica determinado por $A, M$ e $f$ vamos usar a notação $B \cong A \ltimes_{f} M$. Usando a associatividade do produto podemos concluir que $f$ deve satisfazer a seguinte igualdade:

$$
f(a \otimes b) c+f(a b \otimes c)=a f(b \otimes c)+f(a \otimes b c) .
$$

Considere duas extensões equivalentes $A \ltimes_{f_{1}} M$ e $A \ltimes_{f_{2}} M$. A comutatividade do diagrama

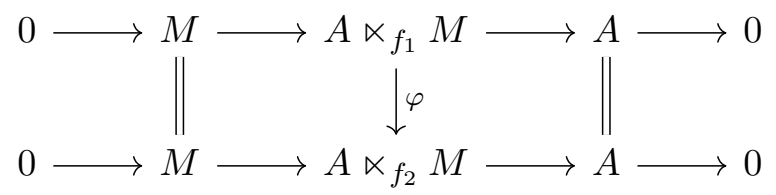

implica $\varphi(a, m)=a, m+g(a)$ para algum $g \in \operatorname{Hom}_{k}(A, M)$. Para $\psi$ ser um homomorfismo de álgebras devemos ter

$$
f_{1}(a \otimes b)-f_{2}(a \otimes b)=a g(b)-g(a b)+g(a) b,
$$

para todo $a, b \in A$.

Como consequência dos argumentos anteriores temos o seguinte Teorema. 
Teorema 7. Seja A uma k-álgebra e $M$ um A-bimódulo. Existe uma correspondência bijetiva entre $H H^{2}(A, M)$ e o conjunto das classes de equivalência de extensões $\operatorname{Ext}(A, M)$ de $A$ por $M$.

Demonstração. Por definição $H H^{2}(A, M)=\operatorname{ker}\left(d^{2}\right) / \operatorname{Im}\left(d^{1}\right)$, onde

$$
\operatorname{Ker}\left(d^{2}\right)=\{f: A \otimes A \rightarrow M: a f(b \otimes c)-f(a b \otimes c)+f(a \otimes b c)-f(a \otimes b) c=0\}
$$

$$
\operatorname{Im}\left(d^{1}\right)=\left\{f: A \otimes A \rightarrow M: f(a \otimes b)=a g(b)-g(a b)+g(a b), g \in H_{k} m_{k}(A, M)\right\}
$$

Portanto, podemos definir uma aplicação sobrejetora

$$
\operatorname{Ker}\left(d^{2}\right) \rightarrow \operatorname{Ext}(A, M)
$$

tal que a imagem de $f \in \operatorname{Ker}\left(d^{2}\right)$ está dada por $A \ltimes_{f} M$. Além disso, $f_{1}-f_{2} \in \operatorname{Im}\left(d^{1}\right)$ se e somente se as extensões $A \ltimes_{f_{1}} M$ e $A \ltimes_{f_{2}} M$ são equivalentes.

Nota 4. Se consideramos álgebras de radical quadrado zero com carcás associado $Q$ conexo, o fato do segundo grupo de cohomologia ser zero fornece condições sobre a forma do carcás $Q$. Enunciaremos isto na Seção 2.5 onde são calculados todos os grupos de cohomologia para álgebras de radical quadrado zero.

\section{$2.3 \quad$ Álgebras hereditárias}

Seja $k$ um corpo algebricamente fechado e $A$ uma $k$-álgebra de dimensão finita, a qual podemos assumir básica e indecomponível. Se $\left\{e_{1}, \ldots, e_{n}\right\}$ é um sistema completo de idempotentes ortogonais primitivos então $\left\{e_{i} \otimes e_{j}\right\}_{1 \leq i, j \leq n}$ é um sistema completo de idempotentes ortogonais primitivos de $A^{e}$. Portanto, $\left\{P(i, j)=\left(e_{i} \otimes e_{j}\right) A^{e} \cong A e_{i} \otimes e_{j} A\right\}$ é um conjunto completo de representantes das classes de isomorfismo de $A^{e}$-módulos projetivos indecomponíveis. Em [Hap89] se apresentam os módulos de uma resolução projetiva minimal que é de utilidade para calcular os grupos de cohomologia de Hochschild de álgebras hereditárias e álgebras da forma $k Q / I \operatorname{com} Q$ um carcás estreito e $I$ um ideal admissível.

Lema 6. [Hap89] Seja

$$
\cdots R_{n} \rightarrow R_{n-1} \rightarrow \cdots \rightarrow R_{1} \rightarrow R_{0} \rightarrow A \rightarrow 0
$$

uma resolução projetiva minimal de $A$ sobre $A^{e}$. Então

$$
R_{n}=\bigoplus_{i, j} P(i, j)^{\operatorname{dim}_{k} E x t_{A}^{n}\left(S_{i}, S_{j}\right)}
$$

onde $P_{i}=e_{i} A$ e $S_{i}=\operatorname{top} P_{i}=P_{i} / \mathrm{rad} P_{i}$.

Definição 24. Dizemos que A é uma álgebra hereditária se os submódulos dos A-módulos projetivos são projetivos.

Lema 7. Seja A uma álgebra. As seguintes afirmações são equivalentes

(a) A é hereditária.

(b) $g l \cdot d i m(A) \leq 1$.

(c) O radical de todo projetivo indecomponivel é projetivo. 
Lema 8. Se A é uma k-álgebra hereditária de dimensão finita existe um carcás $Q$ finito e conexo (sem ciclos) tal que $A \cong k Q$.

Proposição 8. [Hap89] Seja $A=k Q$ uma álgebra básica indecomponivel hereditária de dimensão finita. Então

$$
\operatorname{dim}_{k} H H^{i}(A)= \begin{cases}1 & \text { se } i=0 \\ 0 & \text { se } i>1, \\ 1-n+\sum_{\alpha \in Q_{1}} \nu(\alpha) & \text { se } i=1 .\end{cases}
$$

onde $\nu(\alpha)=\operatorname{dim}_{k} o(\alpha) k Q t(\alpha), n=\left|Q_{0}\right|$ e o $(\alpha) k Q t(\alpha)$ é o subespaço de A gerado pelos caminhos de $o(\alpha)$ a $t(\alpha)$.

Demonstração. Como $Q$ é conexo e não tem ciclos orientados então $H H^{0}(A)=Z(A)=k$.

Pelo Lema (7) $g l \cdot \operatorname{dim}(A) \leq 1$ e $R_{n}$ definido como em (2.5) é nulo para $n \geq 2$. Logo, $H H^{i}(A)=0$ para $i \geq 2$ e $0 \rightarrow R_{1} \rightarrow R_{0} \rightarrow A \rightarrow 0$ é uma resolução projetiva minimal de $A$ sobre $A^{e}$, com

$$
R_{0}=\bigoplus_{i \in Q_{0}} P(i, i) \quad e \quad R_{1}=\bigoplus_{\alpha \in Q_{1}} P(o(\alpha), s(\alpha)),
$$

pois $\operatorname{dim}_{k} \operatorname{Ext}_{A}^{1}\left(S_{i}, S_{j}\right)$ coincide com o número de flechas de $i$ até $j$.

Aplicando $\operatorname{Hom}_{A^{e}}(\cdot, A)$ à sequência anterior obtemos

$$
0 \rightarrow \operatorname{Hom}_{A^{e}}(A, A) \rightarrow \operatorname{Hom}_{A^{e}}\left(R_{0}, A\right) \rightarrow \operatorname{Hom}_{A^{e}}\left(R_{1}, A\right) \rightarrow 0 .
$$

Usando os isomorfismos

$$
\begin{gathered}
\operatorname{Hom}_{A^{e}}(A, A) \simeq k, \\
\operatorname{Hom}_{A^{e}}\left(R_{0}, A\right) \simeq \bigoplus_{i \in Q_{0}} e_{i} A e_{i} \simeq k^{n}, \\
\operatorname{Hom}_{A^{e}}\left(R_{1}, A\right) \simeq \bigoplus_{\alpha \in Q_{1}} o(\alpha) A t(\alpha),
\end{gathered}
$$

podemos concluir que $\operatorname{dim}_{k} H H^{1}(A)=1-n+\sum_{\alpha \in Q_{1}} \operatorname{dim}_{k} O(\alpha) k Q t(\alpha)$.

Corolário 1. Seja $A=k Q$, com $Q$ sem ciclos. Então, $H H^{1}(A)=0$ se e somente se $Q$ é uma árvore.

Exemplo 10. Seja $A=T_{n}(k)$ a álgebra de matrizes triangulares superiores $n \times n$ sobre $k$. Então, A é uma álgebra hereditária com carcás associado

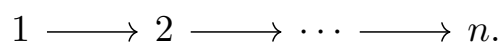

Logo, $H H^{0}(A)=k$ e $H H^{i}(A)=0$ para todo $i>0$.

Nota 5. Existe uma generalização do resultado obtido na Proposição 8 no caso das álgebras truncadas, isto é, álgebras da forma $A=k Q / J^{n}$, com $J$ o ideal gerado pelas flechas e $n \geq 2$ [BLM00].

\subsection{Carcases estreitos}

Definição 25. Seja A uma álgebra de dimensão finita sobre um corpo algebricamente fechado $k$

- Dizemos que $A$ é schuriana se $\operatorname{dim}_{k}\left(\operatorname{Hom}_{A}\left(P, P^{\prime}\right)\right) \leq 1$ para quaisquer $P, P^{\prime}$ A-módulos projetivos indecomponiveis. 
- Dizemos que A é semicomutativa se dados dois caminhos paralelos w e w' em $Q$ tal que $w \in I$ ent $\tilde{a} o w^{\prime} \in I$.

Note que se assumimos $A$ schuriana e semicomutativa então $\nu(\alpha)=1$ para todo $\alpha \in Q .(\nu$ foi definida na Proposição 8.)

Teorema 8. Sejam $Q$ um carcás conexo e finito sem ciclos, I um ideal bilateral de $k Q$ tal que $I \subseteq F^{2}$, e $A=k Q / I$ schuriana e semicomutativa. Então $H H^{0}(A)=k, H H^{1}(A)=1-\left|Q_{0}\right|+\left|Q_{1}\right|$ e $H H^{i}(A)=0$ para todo $i>1$.

Demonstração. Como $Q$ não contém ciclos orientados $H H^{0}(A)=Z(A)=k$.

Note que $\operatorname{dim}_{k}\left(\operatorname{Ext}_{A}^{1}\left(S_{i}, S_{j}\right)\right) \leq 1$ para quaisquer $S_{i}$ e $S_{j} A$-módulos simples. Como $\operatorname{Ext} t_{A}^{1}\left(S_{i}, S_{j}\right)$ fornece o número de flechas de $i$ até $j$ então $\operatorname{Ext}_{A}^{1}\left(S_{i}, S_{j}\right) \neq 0$ se e somente se existe uma flecha $\alpha: i \rightarrow j$.

Se $\operatorname{Ext}_{A}^{t}\left(S_{i}, S_{j}\right) \neq 0$ para $t \geq 2$, então $P_{j}$ aparece numa resolução projetiva minimal de $S_{i}, \operatorname{logo}$ existe um caminho $w$ de $i$ até $j$. Portanto $e_{j} A e_{i}=0$ e o cálculo dos grupos de cohomologia de $A$ se reduz ao cálculo da cohomologia do seguinte complexo

$$
0 \rightarrow \operatorname{Hom}_{A^{e}}(A, A) \rightarrow \operatorname{Hom}_{A^{e}}\left(R_{0}, A\right) \rightarrow \operatorname{Hom}_{A^{e}}\left(R_{1}, A\right) \rightarrow 0 .
$$

Os isomorfismos dados na demonstração do Lema(6) continuam valendo, mas pelas hipóteses $\nu(\alpha)=$ 1 para toda $\alpha \in Q_{1}$ e portanto $\operatorname{dim}_{k}\left(H H^{1}(A)\right)=1-\left|Q_{0}\right|+\sum_{\alpha \in Q_{1}} \nu(\alpha)=1-\left|Q_{0}\right|+\left|Q_{1}\right|$.

Dado um carcás $Q$ o valor $\chi(Q)=1-\left|Q_{0}\right|+\left|Q_{1}\right|$ é conhecido como característica de Euler de $Q$.

Definição 26. Seja $Q$ um carcás conexo e finito. Dizemos que $Q$ é estreito se existe no máximo um caminho entre quaisquer dois vértices.

Corolário 2. Seja $Q$ um carcás estreito e $I$ um ideal admissivel de $k Q$. Então $H H^{0}(k Q / I)=k$, $\operatorname{dim}_{k}\left(H H^{1}(k Q / I)\right)=\chi(Q)$ e $H H^{i}(k Q / I)=0$ para $i>1$.

\section{5 Álgebras de radical quadrado zero}

Teorema 9. (Wedderburn-Malcev)[DK12] Seja A uma k-álgebra associativa de dimensão finita, com $k$ um corpo. Se $A / \operatorname{rad}(A)$ é separável então existe uma subálgebra E semi-simples tal que $A=E \oplus \operatorname{rad}(A)$.

Por simplicidade, ao longo desta seção vamos notar por $r$ o radical de Jacobson de $A$.

Lema 9. [Cib90] Seja A uma k-álgebra de dimensão finita tal que $A / r$ é separável. Seja $A=E \oplus r$ como na decomposição dada no Teorema anterior. A seguinte sequência é uma resolução projetiva de A como A-bimódulo.

$$
\cdots \rightarrow A \otimes_{E} r^{\oplus_{E} i} \otimes_{E} A \stackrel{b_{i}^{\prime}}{\rightarrow} \cdots \rightarrow A \otimes_{E} r \otimes_{E} A \stackrel{b_{1}^{\prime}}{\rightarrow} A \otimes_{E} A \stackrel{\mu}{\rightarrow} A \rightarrow 0,
$$

onde $\mu(a \otimes b)=a b e$

$$
\begin{aligned}
b_{i}^{\prime}\left(a \otimes a_{1} \otimes \cdots \otimes a_{i} \otimes b\right)= & a a_{1} \otimes \cdots \otimes a_{i} \otimes b \\
& +\sum_{j=1}^{i}(-1)^{j} a \otimes a_{1} \otimes \cdots \otimes a_{i} a_{i+1} \otimes \cdots \otimes a_{i} \otimes b \\
& +(-1)^{i} a \otimes a_{1} \otimes \cdots \otimes a_{i} b
\end{aligned}
$$


para todo $a, b \in A$ e todo $a_{1}, \ldots, a_{i} \in r$.

Seja $M$ um $A$-bimódulo . Aplicando o funtor $\operatorname{Hom}_{A-A}(\cdot, M)$ à resolução dada no Lema anterior, e usando o isomorfismo

$$
\operatorname{Hom}_{A-A}\left(A \otimes_{E} X \otimes_{E} A, M\right) \simeq \operatorname{Hom}_{E-E}(X, M),
$$

para todo $E$-bimódulo $X$, podemos calcular a cohomologia de Hochschild como a cohomologia do complexo

$$
\begin{array}{r}
0 \rightarrow M^{E} \stackrel{b_{1}}{\rightarrow} \operatorname{Hom}_{E-E}(r, M) \stackrel{b_{2}}{\longrightarrow} \operatorname{Hom}_{E-E}\left(r \otimes_{E} r, M\right) \rightarrow \cdots \\
\cdots \rightarrow \operatorname{Hom}_{E-E}\left(r^{\otimes_{E} i}, M\right) \stackrel{b_{i+1}}{\longrightarrow} \cdots,
\end{array}
$$

onde $M^{E}=\{m \in M \mid s m=m s, \forall s \in E\},(d m)(x)=m x-x m$ para todo $m \in M^{E}$ e todo $x \in r$, e

$$
\begin{aligned}
\left(b_{i+1} f\right)\left(x_{1} \otimes \cdots \otimes x_{i+1}\right)= & x_{1} f\left(x_{2} \otimes \cdots \otimes x_{i+1}\right) \\
& +\sum(-1)^{j} f\left(x_{1} \otimes \cdots \otimes x_{j} x_{j+1} \otimes \cdots \otimes x_{i+1}\right) \\
& +(-1)^{i+1} f\left(x_{1} \otimes \cdots \otimes x_{i}\right) x_{i+1}
\end{aligned}
$$

para todo $f \in \operatorname{Hom}_{E-E}\left(r^{\otimes_{E} i, M}\right)$ e todo $x_{1}, \ldots, x_{i+1} \in r$.

Dado um corpo $k$ e uma $k$-álgebra básica $A$ de dimensão finita tal que $(\operatorname{rad} A)^{2}=0$, existe um carcás $Q$ finito e conexo tal que $A \cong k Q / F^{2}$, onde $F$ é o ideal gerado pelas flechas em $Q$. Neste caso, dizemos que $A$ é de radical quadrado zero. Para álgebras de radical quadrado zero os homomorfismos do complexo anterior ficam definidos simplesmente por

$$
\left(b_{i+1} f\right)\left(x_{1} \otimes \cdots \otimes x_{i+1}\right)=x_{1} f\left(x_{2} \otimes \cdots x_{i+1}\right)+(-1)^{i+1} f\left(x_{1} \otimes \cdots \otimes x_{i}\right) x_{i+1} .
$$

Toda álgebra $A$ de dimensão finita com radical quadrado zero é Morita equivalente a uma álgebra da forma $(k Q)_{2}=k Q_{0} \oplus k Q_{1}$ com produto dado por $(e, x) \cdot\left(e^{\prime}, x^{\prime}\right)=\left(e e^{\prime}, e x^{\prime}+x e^{\prime}\right)$. Além disso, $\operatorname{rad}(k Q)_{2}=k Q_{1}$ e $E=k Q_{0}$ é uma subálgebra maximal semi-simples separável.

A seguir apresentamos uma serie de resultados que permitem calcular os grupos de cohomologia de Hochschild de toda álgebra de dimensão finita de radical quadrado zero. Estes resultados podem ser encontrados em [Cib98]. Além disso, usaremos a mesma notação do artigo referenciado.

Lema 10. Seja $r=k Q_{1}$ o radical de $\left(k Q_{2}\right)$ e $E=k Q_{0}$. Então, $r^{\otimes_{E} n}$ tem uma base dada por $Q_{n}$, o conjunto de caminhos de comprimento $n$.

Dados dois conjuntos de caminhos $X$ e $Y$ vamos notar por $X / / Y$ o conjunto de duplas $\left(\gamma, \gamma^{\prime}\right) \in$ $X \times Y$ tais que $o(\gamma)=o\left(\gamma^{\prime}\right)$ e $t(\gamma)=t\left(\gamma^{\prime}\right)$.

Lema 11. Seja $A=(k Q)_{2}$. O espaço vetorial $H_{o m-E}\left(r^{\otimes_{E} n}, A\right)$ é isomorfo a $k\left(Q_{n} / / Q_{0}\right) \oplus k\left(Q_{n} / /\right.$ $\left.Q_{1}\right)$.

Proposição 9. O seguinte diagrama comuta para todo $n$

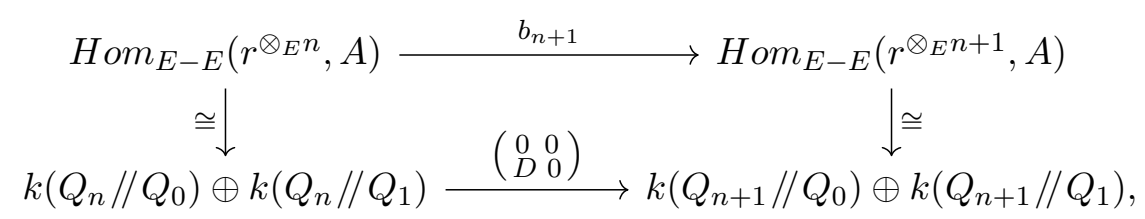


com $D: k\left(Q_{n} / / Q_{0}\right) \rightarrow k\left(Q_{n+1} / / Q_{1}\right)$ dado por

$$
D(\gamma, e)=\sum_{a \in e Q_{1}}(\gamma a, a)+(-1)^{n+1} \sum_{a \in Q_{1} e}(a \gamma, a) .
$$

Teorema 10. Seja $Q$ um carcás conexo tal que $Q$ não é um ciclo. Então

$$
\operatorname{dim}_{k} H H^{n}\left((k Q)_{2}\right)= \begin{cases}1+\left|Q_{1}\right|\left|Q_{0}\right| & \text { se } n=0, \\ \left|Q_{1}\right|\left|Q_{1}\right|-\left|Q_{0}\right|\left|Q_{0}\right|+1 & \text { se } n=1, \\ \left|Q_{n}\right|\left|Q_{1}\right|-\left|Q_{n-1}\right|\left|Q_{0}\right| & \text { se } n>1 .\end{cases}
$$

Demonstração. Vamos mostrar que no caso em que $Q$ não é um ciclo o homomorfismo $D: k\left(Q_{n} / /\right.$ $\left.Q_{0}\right) \rightarrow k\left(Q_{n+1} / / Q_{1}\right)$ é injetivo para $n>0$. Seja $x \in \operatorname{Ker}(D)$. Podemos expressar $x$ da forma

$$
x=\sum_{(\gamma, e) \in k\left(Q_{n} / / Q_{0}\right)} x_{(\gamma, e)}(\gamma, e) .
$$

Seja $(\gamma, e)$ um ciclo fixo na expressão (2.6). Então,

$$
D\left(x_{(\gamma, e)}(\gamma, e)\right)=x_{(\gamma, e)}\left[\sum_{a \in e Q_{1}}(\gamma a, a)+(-1)^{n+1} \sum_{a \in Q_{1} e}(a \gamma, a)\right] .
$$

Vamos notar por $f(\gamma)$ a primeira flecha de $\gamma$ e por $l(\gamma)$ a ultima flecha de $\gamma$.

- Afirmação 1. Se existe uma flecha $a$ tal que $a \neq f(\gamma), a \neq l(\gamma)$ e $o(a)=e$ ou $t(a)=e$, então $x_{(\gamma, e)}=0$.

Seja $a \in Q_{1}$ tal que $o(a)=e$ e $a \neq f(\gamma)$, então $(\gamma a, a)$ não está no suporte de $D\left(\gamma^{\prime}, e^{\prime}\right)$ para todo $\left(\gamma^{\prime}, e^{\prime}\right) \neq(\gamma, e)$. De fato, suponha que $(\gamma a, a)=\left(\gamma^{\prime} a^{\prime}, a^{\prime}\right)$, então $a=a^{\prime}$ e $\gamma=\gamma^{\prime}$. Se $(\gamma a, a)=\left(a^{\prime} \gamma^{\prime}, a^{\prime}\right)$, então $a=a^{\prime}$ e $\gamma a=a \gamma^{\prime}, \operatorname{logo} a=f(\gamma)$, contrário do que foi suposto.

Analogamente, se $a \in Q_{1}$ é tal que $t(a)=e$ e $a \neq l(\gamma)$, então $(\gamma a, a)$ não está no suporte de $D\left(\gamma^{\prime}, e^{\prime}\right)$ para todo $\left(\gamma^{\prime}, e^{\prime}\right) \neq(\gamma, e)$.

Agora, vamos supor que não existe $a \in Q_{1}$ satisfazendo as condições da afirmação anterior, isto é, $e Q_{1}=\{f(\gamma)\}$ e $Q_{1} e=\{l(\gamma)\}$.

Se $\gamma=f(\gamma) a_{2} \cdots a_{j} l(\gamma)$, notamos por $(\bar{\gamma}, \bar{e})$ o elemento em $Q_{n} / / Q_{0}$ tal que $\bar{\gamma}=a_{2} \cdots a_{j} l(\gamma) f(\gamma)$ e $\bar{e}=o\left(a_{2}\right)=t(f(\gamma))$

- Afirmação 2. $x_{(\gamma, e)}=(-1)^{n} x_{(\bar{\gamma}, \bar{e})}$.

$(\gamma f(\gamma), f(\gamma)) \in \operatorname{supp}(D(\gamma, e))$ e como $(f(\gamma) \bar{\gamma}, f(\gamma))=(\gamma f(\gamma), f(\gamma))$ então $(\gamma f(\gamma), f(\gamma)) \in$ $\operatorname{supp}(D(\bar{\gamma}, \bar{e}))$. Vamos ver que $(\gamma f(\gamma), f(\gamma))$ não está em outro suporte. Seja $\left(\gamma^{\prime}, e^{\prime}\right)$ ciclo tal que existe $a \in e^{\prime} Q_{1}$ e $\left(\gamma^{\prime} a, a\right)=(\gamma f(\gamma), f(\gamma))$, então $a=f(\gamma), \gamma^{\prime} f(\gamma)=\gamma f(\gamma)$, logo $\gamma^{\prime}=\gamma$. Analogamente, se $\left(\gamma^{\prime}, e^{\prime}\right)$ é um ciclo tal que $a \in Q_{1} e^{\prime}$ e $\left(a \gamma^{\prime}, a\right)=(\gamma f(\gamma), f(\gamma))$ então $a=f(\gamma)$ e $\gamma^{\prime}=\bar{\gamma}$. Portanto, o coeficiente de $(\gamma f(\gamma), f(\gamma))$ em $D(x)$ é $x_{(\gamma, e)}+(-1)^{n+1} x_{(\bar{\gamma}, \bar{e})}$. Como $D(x)=0$ então $x_{(\gamma, e)}+(-1)^{n+1} x_{(\bar{\gamma}, \bar{e})}=0$.

Sabemos que $\gamma=a_{1} a_{2} \ldots a_{j} a_{j+1}$, com $a_{1}=f(\gamma)$ e $a_{j+1}=l(\gamma)$. Como $Q$ é conexo e não é um ciclo existe $1 \leq k \leq j$ minimal tal que existe $a \in Q_{1}$ que satisfaz $a \neq a_{k+1}, a \neq a_{k}$ e $o(a)=$ $o\left(a_{k+1}\right)=t\left(a_{k}\right)$ ou $t(a)=o\left(a_{k+1}\right)=t\left(a_{k}\right)$. Pela Afirmação 1 temos que $x_{\left(a_{k+1} a_{k+2} \ldots a_{k}, o\left(a_{k+1}\right)\right)}=0$. Pela minimalidade de $k$, então $x_{(\gamma, e)}=(-1)^{n} x_{(\bar{\gamma}, \bar{e})}=\cdots=(-1)^{k n} x_{\left(a_{k+1} a_{k+2} \ldots a_{k}, o\left(a_{k+1}\right)\right)}=0$.

Acabamos de mostrar que $x_{(\gamma, e)}=0$ para todo $(\gamma, e)$ na expressão $(2.6)$, logo $x=0$ e está mostrada a injetividade de $D$. 
Para o caso $n=0$ o homomorfismo $D: k\left(Q_{0} / / Q_{0}\right) \rightarrow k\left(Q_{1} / / Q_{1}\right)$ fica definido por

$$
D(e, e)=\sum_{a \in e Q_{1}}(a, a)-\sum_{a \in Q_{1} e}(a, a)
$$

para todo $e \in Q_{0}$. Logo, se $x=\sum_{i} \mu_{i}\left(e_{i}, e_{i}\right) \in \operatorname{Ker}(D)$ então,

$$
0=\sum_{i} \mu_{i}\left[\sum_{a \in e_{i} Q_{1}}(a, a)-\sum_{a \in Q_{1} e_{i}}(a, a)\right]=\sum_{a \in Q_{1}}\left(\mu_{o(a)}-\mu_{t(a)}\right)(a, a) .
$$

Logo, $\mu_{o(a)}=\mu_{t(a)}$ para toda $a \in Q_{1}$. Então, pela conexidade de $Q, \mu_{i}=\mu_{j}$ para todo $i, j$ e portanto $\operatorname{Ker}(D)=\left\langle\sum_{e \in Q_{0}}(e, e)\right\rangle$.

O anterior e a injetividade de $D$ para $n>0$ mostra o resultado.

Corolário 3. Seja $Q$ um carcás conexo tal que $Q$ não é um ciclo. Então $\bigoplus_{n \geq 0} H H^{n}\left((k Q)_{2}\right)$ é um espaço vetorial de dimensão finita se e somente se $Q$ não tem ciclos. Mais precisamente, se $Q$ tem um ciclo orientado de comprimento $c$, então $H H^{c n+1}\left((k Q)_{2}\right) \neq 0$ para todo $n>0$.

Proposição 10. Seja $Q$ um ciclo de comprimento $m \geq 2$. Então $H H^{0}\left((k Q)_{2}\right)=Z\left((k Q)_{2}\right)=k e$

(i) Se char $(k) \neq 2, H H^{n}\left((k Q)_{2}\right)=H H^{n+1}\left((k Q)_{2}\right)=k$ para $n$ par múltiplo de $m$. Nos outros casos a cohomologia é zero.

(ii) Se char $(k)=2, H H^{n}\left((k Q)_{2}\right)=H H^{n+1}\left((k Q)_{2}\right)=k$ para todo $n$ múltiplo de $m$. Nos outros casos a cohomologia é zero.

Proposição 11. Seja $Q$ um laço. Então $A=(k Q)_{2}=k[x] /\left\langle x^{2}\right\rangle e$

(i) $\operatorname{Se} \operatorname{char}(k) \neq 2, H H^{0}(A)=A$ e $H H^{n}(A)=k$ para $n>0$.

(ii) $\operatorname{Se} \operatorname{char}(k)=2, H H^{n}(A)=A$ para todo $n \geq 0$.

Corolário 4. Seja $A=(k Q)_{2}$ uma k-álgebra de dimensão finita de radical quadrado zero. As seguintes afirmações são equivalentes:

(i) $H H^{i}(A)=0$ para $i>0$.

(ii) $H H^{1}(A)=0$.

(iii) $Q$ é uma árvore.

Demonstração. (ii $) \Rightarrow($ iii $)$ Se $H H^{1}(A)=0$, então $Q$ não é um ciclo e $\left|Q_{1} / / Q_{1}\right|=\left|Q_{0} / / Q_{0}\right|-1$, $\operatorname{logo}\left|Q_{1}\right| \leq\left|Q_{0}\right|-1$, mas $\left|Q_{1}\right|$ não pode ser menor estrito a $\left|Q_{0}\right|-1$, $\log Q$ é uma árvore.

$($ iii $) \Rightarrow\left(\right.$ (i) Se $Q$ é uma árvore, então $\left|Q_{1}\right|=\left|Q_{0}\right|-1$ e o grafo subjacente não tem ciclos. Pelo Teorema (10) $H H^{i}(A)=0$ para todo $i>0$.

Nota 6. Em [Hap89] Happel faz uma demonstração do Corolário(4) usando derivações.

Proposição 12. Seja $Q$ um carcás conexo. Então $H H^{2}\left((k Q)_{2}\right)=0$ se e somente se $Q$ não tem laços, não é um ciclo de comprimento 2 e não contém triângulos não orientados, isto é,

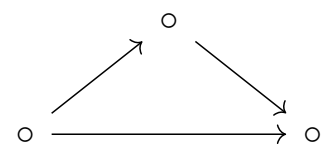




\section{Capítulo 3}

\section{Resolução projetiva para Álgebras associativas}

Este capítulo expõe os conceitos e resultados apresentados em [CS15]. Dada uma álgebra da forma $k Q / I$ com $Q$ um carcás com um número finito de vértices e $I$ um ideal bilateral definimos um sistema de redução que junto com uma ordem sobre os conjuntos dos caminhos satisfazendo a condição da cadeia descendente e compatível com a concatenação, serão úteis para construir uma $k$-base da álgebra sob condições especiais. Tendo um sistema de redução $\mathcal{R}$ que satisfaz a condição do Diamante para $I$ definimos as $n$-ambiguidades, necessárias para construir a resolução projetiva de $A$ como bimódulo. As ideias usadas para a construção desta resolução estão baseadas na Teoria das Bases de Gröbner e é uma generalização da resolução projetiva para álgebras monomiais exibida por Bardzell [Bar97].

\subsection{Sistemas de Redução}

Seja $k$ um corpo e $Q$ carcás com um número finito de vértices. Vamos notar por $Q_{\geq n}$ o conjunto de caminhos de comprimento maior ou igual a $n$. Note que $Q_{\geq 0}$ é simplesmente o conjunto de todos os caminhos em $Q$, incluindo os vértices os quais consideramos como caminhos de comprimento zero.

Seja $E:=k Q_{0}$ a subálgebra da álgebra de caminhos gerada pelos vértices em $Q$.

Definição 27. Sejam $a, b, p, q$ caminhos em $Q$. Se $q=a p b$ dizemos que $p$ é um divisor de $q$. No caso em que $a \in Q_{0}$ (resp. $b \in Q_{0}$ ) dizemos que $p$ é um divisor à esquerda (resp. divisor à direita) de $q$.

A seguir apresentamos uma série de definições que vamos precisar ao longo deste capítulo.

- Um conjunto $\mathcal{R} \subseteq Q_{\geq 0} \times k Q$ é chamado sistema de redução se para todo $(s, f) \in \mathcal{R}, f$ é paralelo a $s$ e $s \neq f$.

- Uma redução básica é uma tripla $(a, \rho, c)$ tal que $\rho=(s, f) \in \mathcal{R}$, e $a$ e $c$ são caminhos tais que $a s c \neq 0$. Toda redução básica determina um endomorfismo de $E$-bimódulos $r_{a, \rho, c}: k Q \rightarrow k Q$ tal que $r_{a, \rho, c}(a s c)=a f c$ e $r_{a, \rho, c}(q)=q$ para todo $q \neq a s c$.

- Um caminho $p$ é de redução finita com respeito a um sistema de redução $\mathcal{R}$ se para toda sequência infinita de reduções básicas $\left(r_{i}\right)_{i \in \mathbb{N}}$, existe $m \in \mathbb{N}$ tal que $r_{n} \circ \cdots \circ r_{1}(p)=r_{m} \circ \cdots \circ$ $r_{1}(p)$ para todo $n \geq m$.

Sejam $p$ e $q$ caminhos em $Q$. Escrevemos $q \rightsquigarrow p$ se $p=q$ ou se existem reduções básicas $r_{1}, \ldots, r_{n}$, com $n \geq 1$, e caminhos $p_{1}, \ldots, p_{n+1}$ tais que $p_{1}=q, p_{n+1}=p$, e $p_{i+1} \in \operatorname{supp}\left(r_{i}\left(p_{i}\right)\right)$ para todo $i=1, \ldots, n$. Note que $\rightsquigarrow$ define uma relação reflexiva e transitiva sobre $Q_{\geq 0}$ 
Lema 12. Seja $\mathcal{R}$ um sistema de redução tal que todo caminho é de redução finita. Então

(1) Se $p$ é um caminho e $r$ é uma redução básica tal que $p \in \operatorname{supp}(r(p))$, então $r(p)=p$.

(2) A relação $\rightsquigarrow$ é uma ordem sobre o conjunto dos caminhos $Q_{\geq 0}$ compativel com a concatenação.

(3) A relação $\rightsquigarrow$ satisfaz a condição da cadeia descendente. No caso em que uma relação satisfaz a condição da cadeia descendente dizemos que ela é Noetheriana.

Demonstração. (1) Como $p \in \operatorname{supp}(r(p))$ então $r(p)=\lambda p+x$, com $\lambda \in K^{*}$ e $p \notin \operatorname{supp}(x)$. Vamos supor que $x \neq 0$ ou $\lambda \neq 1$, então $r(p) \neq p$ e como $r$ é uma redução básica então ela fixa qualquer caminho diferente de $p, \operatorname{logo} r(x)=x$, pois $p \notin \operatorname{supp}(x)$.

Seja $(r, r, \ldots)$ uma sequência infinita de reduções. Pela hipótese, existe $k \in \mathbb{N}$ tal que $r^{k}(p)=r^{k+1}(p)$, isto é,

$$
\lambda^{k} p+\left(\sum_{i=0}^{k-1} \lambda^{i}\right) x=\lambda^{k+1} p+\left(\sum_{i=0}^{k} \lambda^{i}\right) x .
$$

O anterior implica $\lambda=1$ e $x=0$, contradizendo o assumido.

(2) Para mostrar que a relação $\rightsquigarrow$ é uma ordem sobre $Q_{\geq 0}$ basta mostrar que ela é antissimétrica. Vamos supor que não é este o caso, então existem $p_{1}, \ldots, p_{n+1}$ caminhos e $r_{1}, \ldots, r_{n}$ reduções básicas tais que $p_{1}=p_{n+1}$ e $p_{i+1} \in \operatorname{supp}\left(r_{i}\left(p_{i}\right)\right)$ para todo $1 \leq i \leq n$. Suponha $n$ minimal. Existem $x_{1}, \ldots, x_{n} \in k Q$ e $\lambda_{1}, \ldots, \lambda_{n} \in K^{*}$ tais que $r_{i}\left(p_{i}\right)=\lambda_{i} p_{i+1}+x_{i}$, com $p_{i+1} \notin \operatorname{supp}\left(x_{i}\right)$. Pela minimalidade de $n, r_{i}\left(p_{i}\right) \neq p_{i}$, então $r_{i}$ fixa qualquer caminho distinto de $p_{i}$, para todo $i$.

Vamos mostrar que $p_{i} \notin \operatorname{supp}\left(x_{j}\right)$ para todo $j<i$. Suponha que existem $i, j$ tais que $p_{i} \in \operatorname{supp}\left(x_{j}\right), \operatorname{com} j<i$. Defina $u_{k}:=p_{k}, t_{k}:=r_{k}$ para $1 \leq k \leq j$ e $u_{j+l}:=p_{i+l-1}$, $t_{j+l}:=r_{i+l-1}$ para $1 \leq l \leq n-i+2$. É fácil ver que $u_{s+1} \in t_{s}\left(u_{s}\right)$ para $1 \leq s \leq j+n-i+2$, contradizendo a minimalidade de $n$. Pelo anterior, $p_{1}=p_{n+1} \notin \operatorname{supp}\left(x_{j}\right)$ para todo $1 \leq j \leq n$ e a partir dos caminhos $p_{1}, \ldots, p_{n+1}$ e as reduções $r_{1}, \ldots, r_{n}$ é possível construir sequências cíclicas onde o primeiro e último termo é igual a $p_{i}$, para cada $i$. Usando o argumento anterior podemos concluir $p_{i} \notin \operatorname{supp}\left(x_{j}\right)$ para todo $i, j$. Isto implica $r_{n} \circ \cdots \circ r_{1}\left(p_{1}\right)=\lambda p_{1}+x$, para algum $\lambda \in K^{*}$ e $x \in k Q$ com $p_{i} \notin \operatorname{supp}(x)$, para todo $i$. Defina a sequência infinita de reduções básicas $\left(r_{i}\right)_{i} \in \mathbb{N}$ por $r_{m}:=r_{[m]_{n}}$. Seja $r:=r_{n} \circ \cdots \circ r_{1}$, note que $r^{l}\left(p_{1}\right)=\lambda^{l} p_{1}+\sum_{i=0}^{l-1} \lambda^{i} x$, logo, a sequência infinita definida anteriormente não se estabiliza em $p_{1}$, contradizendo que todo caminho é de redução finita.

Agora vamos mostrar que $\rightsquigarrow$ é compatível com a concatenação. Se $p$ e $q$ são tais que $p=q$, o resultado é imediato. Sejam $p$ e $q$ caminhos distintos, e $r$ redução básica tal que $p \in \operatorname{supp}(r(q))$ e $r=r_{a^{\prime}, \rho, c^{\prime}}, \operatorname{com} \rho=(s, f) \in \mathcal{R}$. Como $r(q) \neq q$ então $q=a^{\prime} s c^{\prime}$ e $r(q)=a^{\prime} f c^{\prime}$. Sejam $a$ e $c$ caminhos tais que $a p c \neq 0$ e $a q c \neq 0$. Sabemos que $p \in \operatorname{supp}(r(q)), \operatorname{logo} p \in \operatorname{supp}\left(a^{\prime} f c^{\prime}\right)$, o que implica apc $\in \operatorname{supp}\left(a a^{\prime} f c^{\prime} c\right)$. Note que $r_{a a^{\prime}, \rho, c^{\prime} c}(a q c)=a a^{\prime} f c^{\prime} c$, portanto $a q c \rightsquigarrow a p c$. O resultado segue pela transitividade de $\rightsquigarrow$.

(3) Suponhamos que existe uma sequência de caminhos $\left(p_{i}\right)_{i \in \mathbb{N}}$ e uma sequência de reduções básicas $\left(r_{i}\right)_{i \in \mathbb{N}}$ tal que $p_{i+1} \in \operatorname{supp}\left(t_{i}\left(p_{i}\right)\right)$ para todo $i \in \mathbb{N}$ Como $\rightsquigarrow$ é antissimétrica 
podemos assumir $p_{i} \neq p_{j}$ para $i \neq j$ e $p_{j} \notin \operatorname{supp}\left(x_{i}\right)$ se $i<j$. Vamos mostrar por indução sobre $k$ que existem $i_{1}, \ldots, i_{k}$, com $1=i_{1}<\cdots<i_{k}$ e

$$
\begin{gathered}
p_{i_{k}} \in \operatorname{supp}\left(t_{i_{k-1}} \circ \cdots \circ t_{1}\left(p_{1}\right)\right) \\
p_{j} \notin \operatorname{supp}\left(t_{i_{k-1}} \circ \cdots \circ t_{1}\left(p_{1}\right)\right), j>i_{k} .
\end{gathered}
$$

Para o caso $k=2$, seja $i_{2}=2$. Então $p_{2} \in \operatorname{supp}\left(t_{1}\left(p_{1}\right)\right)$ e $t_{1}\left(p_{1}\right)=\lambda_{1} p_{2}+x_{1}$, com $\lambda_{1} \in K^{*}$ e $p_{2} \notin \operatorname{supp}\left(x_{1}\right)$. Para todo $j>2, p_{j} \notin \operatorname{supp}\left(x_{1}\right), \operatorname{logo} p_{j} \notin \operatorname{supp}\left(t_{1}\left(p_{1}\right)\right)$ para todo $j>2$.

Suponhamos que existem $1<i_{2}<\cdots<i_{k}$ e $p_{i_{k}}$ tais que são satisfeitas as condições (3.1) e (3.2). Seja

$$
X_{k}=\left\{i>i_{k} \mid p_{i} \in \operatorname{supp}\left(t_{i_{k}} \circ \cdots \circ t_{i_{1}}\left(p_{1}\right)\right)\right\} .
$$

Pela hipótese de indução, $t_{i_{k-1}} \circ \cdots \circ t_{i_{1}}\left(p_{1}\right)=\lambda p_{i_{k}}+x$, com $\lambda \in K^{*}, x \in k Q$ e $p_{i_{k}} \notin$ $\operatorname{supp}(x)$. Pela construção da sequência $p_{i_{k+1}} \in \operatorname{supp}\left(t_{i_{k}}\left(p_{i_{k}}\right)\right)$ e $p_{i_{k+1}} \notin \operatorname{supp}\left(t_{i_{k-1}} \circ \cdots \circ\right.$ $\left.t_{i_{1}}\left(p_{1}\right)\right), \operatorname{logo} p_{i_{k+1}} \notin \operatorname{supp}(x)$, então $p_{i_{k+1}} \in \operatorname{supp}\left(t_{i_{k}}\left(p_{i_{k}}\right)+x\right)$. Como $t_{i_{k}} \circ \cdots \circ t_{i_{1}}\left(p_{1}\right)=$ $\lambda t_{i_{k}}\left(p_{i_{k}}\right)+t_{i_{k}}(x)=\lambda t_{i_{k}}\left(p_{i_{k}}\right)+x$, então $p_{i_{k+1}} \in \operatorname{supp}\left(t_{i_{k}} \circ \cdots \circ t_{i_{1}}\left(p_{1}\right)\right.$. Portanto, $X_{k} \neq \emptyset$ e podemos definir $i_{k+1}=\max X_{k}$.

Definição 28. Uma redução é uma $n$-upla $\left(r_{n}, \ldots, r_{1}\right)$ com $n \in \mathbb{N}$ e $r_{i}$ uma redução básica para $1 \leq i \leq n$. Toda redução $r=\left(r_{n}, \ldots, r_{1}\right)$ determina um endomorfismo $r: k Q \rightarrow k Q$ de E-bimódulos, com $r=r_{n} \circ \cdots \circ r_{1}$.

Lema 13. Se $p$ e q são caminhos, então $q \rightsquigarrow p$ se e somente se $p=q$ ou existe uma redução $r$ tal que $p \in \operatorname{supp}(r(q))$.

Definição 29. Seja I um ideal bilateral de $k Q$. Dizemos que um sistema de redução $\mathcal{R}$ satisfaz a condição do Diamante para I se:

(1) I é o ideal bilateral gerado pelo conjunto $\{(s-f)\}_{(s, f) \in \mathcal{R}}$,

(2) Todo caminho é de redução única, e

(3) Para todo $(s, f) \in \mathcal{R}, f$ é irredutivel.

Seja $\leq$ uma boa ordem sobre $Q_{0} \cup Q_{1}$, e seja $\omega: Q_{1} \rightarrow \mathbb{N} \backslash\{0\}$. Podemos estender $\omega$ a $k Q$ fazendo $\omega(v)=0$ para todo $v \in Q_{0}$ e se $p \in Q_{\geq 0}$, com $p=a_{1} \cdots a_{m}$, com $a_{i} \in Q_{1}$ para todo $1 \leq i \leq m$, então $\omega\left(a_{1} \cdots a_{m}\right)=\sum_{i=1}^{m} \omega\left(a_{i}\right)$. Sejam $p, q \in Q_{\geq 0}$. Escrevemos $p \leq_{\omega} q$ se :

- $\omega(p)<\omega(q)$ ou

- $p, q \in Q_{0}$ e $p \leq q$ ou

- $\omega(p)=\omega(q), p=a_{1} \cdots a_{n}, q=b_{1} \cdots b_{m} \in Q_{\geq 1}$ e existe $j \leq \min (|p|,|q|)$ tal que $a_{i}=b_{i}$ para $i<j$ e $a_{j}<b_{j}$.

A ordem $\leq_{\omega}$ é chamada ordem peso-lexicográfica e com ela vamos conseguir mostrar que dado um ideal bilateral $I$ sempre existe um sistema de redução que satisfaz a condição do Diamante para I. Esta ordem é compatível com a concatenação e dado um caminho $p$ o conjunto de caminhos menores a $p$ com relação a esta ordem é finito.

Notação: Seja $p \in k Q$. Se $p=\sum_{i=1}^{n} \lambda_{i} p_{i}$, com $\lambda_{i} \in k^{*}, p_{i} \in Q_{\geq 0}$ para todo $1 \leq i \leq n$, notamos por tip $(p)$ o maior elemento do conjunto $\left\{p_{i}\right\}_{i=1}^{n}$ com relação a ordem $\leq_{\omega}$. Dado $X \subseteq k Q$, notamos por $\operatorname{tip}(X)$ o conjunto $\{\operatorname{tip}(x) \mid x \in X\}$. 
Dado um conjunto $X$ notamos por $k\langle X\rangle$, a $k$-álgebra associativa livre sobre $X$ e por $\langle X\rangle$, o semigrupo livre com unidade sobre $X$.

Mais diante definiremos o conceito de ambiguidade o qual sera útil para construir a resolução projetiva desejada. A seguir apresentamos um Teorema que envolve este conceito e permite demonstrar a existência de um sistema de redução que satisfaz a condição do Diamante dado um ideal bilateral de $k Q$.

Teorema 11. Seja $X$ um conjunto, $\mathcal{R}$ um sistema de redução para $k\langle X\rangle$ e $\leq$ uma ordem parcial sobre $\langle X\rangle$ que satisfaz a condição da cadeia descendente para todo $(s, f) \in \mathcal{R}$ e se $c \in \operatorname{supp}(f)$ então $s>c$. Além disso, se $b<b^{\prime}$ então $a b c<a b^{\prime} c$ para quaisquer $b, b^{\prime}, a, c \in\langle X\rangle$. As seguintes afirmações são equivalentes:

1. Todas as ambiguidades de $\mathcal{R}$ são resolvíveis.

2. Todos os elementos em $k\langle X\rangle$ são de redução única.

3. O conjunto das classes dos caminhos irredutiveis é uma $k$-base de $k Q / I$, onde $I$ é o ideal bilateral gerado pelo conjunto $\{s-f \mid(s, f) \in \mathcal{R}\}$.

Proposição 13. Se I é um ideal bilateral de $k Q$, existe um sistema de redução $\mathcal{R}$ que satisfaz a condição do Diamante para I (Definição 29).

Demonstração. Seja $\leq$ uma boa ordem sobre $Q_{0} \cup Q_{1}$, e $\omega: Q_{1} \rightarrow \mathbb{N} \backslash\{0\}$ uma função peso. Considere a ordem total $\leq_{\omega}$ peso-lexicográfica sobre $Q_{\geq 0}$. Seja

$$
S:=\operatorname{Mintip}(I)=\left\{p \in \operatorname{tip}(I) \mid p^{\prime} \notin \operatorname{tip}(I) \text { se } p^{\prime} \text { é divisor próprio de } p\right\} \text {. }
$$

Para cada $s \in S, s=t i p(x)$ para algum $x \in I$, então podemos escolher $f_{s} \in k Q$ paralelo a $s$, tal que $f_{s}<_{\omega} s$ e $s-f_{s} \in I$.

Vamos mostrar primeiro que o sistema de redução $\mathcal{R}=\left\{\left(s, f_{s}\right)\right\}_{s \in S}$ é tal que $I=\left\langle s-f_{s}\right\rangle_{s \in S}$ e todo caminho é de redução única.

A inclusão $\left\langle s-f_{s}\right\rangle_{s \in S} \subseteq I$ é clara pela escolha dos $f_{s}$. Seja $x \in I$, então podemos escrever $x$ da forma $\sum_{i=1}^{n} \lambda_{i} c_{i}$, com $\operatorname{tip}(x)=c_{1}$. Existe $s \in S$ tal que $s$ divide $c_{1}$, isto é, existem $a, c \in Q_{\geq 0}$ tais que $a s c=c_{1}$.

Seja $x^{\prime}=\lambda_{1} a f_{s} c+\sum_{i=2}^{n} \lambda_{i} c_{i}, \operatorname{logo} x=\lambda_{1} a\left(s-f_{s}\right) c+x^{\prime}$, o que implica $x^{\prime} \in I$. Note que $c_{1}>_{\omega}$ tip $\left(x^{\prime}\right)$. Se $x^{\prime}=0$ claramente $x \in\left\langle s-f_{s}\right\rangle_{s \in S}$, caso contrário, podemos repetir o processo anterior obtendo $x^{\prime \prime} \in k Q$ com tip $\left(x^{\prime}\right)>_{\omega} \operatorname{tip}\left(x^{\prime \prime}\right)$ e $x=\lambda_{1} a\left(s-f_{s}\right) c+\lambda_{1}^{\prime} a^{\prime}\left(s^{\prime}-f_{s}^{\prime}\right) c^{\prime}+x^{\prime \prime}$. Como o conjunto $\left\{p \in Q_{\geq 0} \mid p<_{\omega} c_{1}\right\}$ é finito este processo só pode ser reiterado um número finito de vezes e portanto $x \in\left\langle s-f_{s}\right\rangle_{s \in S}$.

Como $s>_{\omega} f_{s}$ então todo caminho é de redução finita pelo fato de $\leq_{\omega}$ ser compatível com concatenação. Dado $p$ um caminho para toda redução $r$ temos que $p-r(p) \in\left\langle s-f_{s}\right\rangle_{s \in S}=I$. Sejam $r$ e $t$ duas reduções tais que $r(p)$ e $t(p)$ são irredutíveis. Logo $r(p)-t(p)=(p-t(p))-(p-r(p)) \in I$. Se $r(p)-t(p) \neq 0$ então $d=t i p(r(p)-t(p))$ é divisível por algum $s \in S$, existem $a, c \in Q_{\geq 0}$ tais que $a s c=d$, e a redução $r_{a, s, c}$ atua de maneira não trivial sobre $r(p)$ ou sobre $t(p)$, obtendo uma contradição.

Pelo anterior, dado $s \in S$, existe uma redução $r$ tal que $r\left(f_{s}\right)=f_{s}^{\prime}$ é irredutível. Considere o sistema de redução $\mathcal{R}^{\prime}=\left\{\left(s, f_{s}^{\prime}\right)\right\}_{s \in S}$. Note que o conjunto $\mathcal{B}$ dos caminhos irredutíveis com relação a $\mathcal{R}$ é o mesmo conjunto $\mathcal{B}^{\prime}$ dos caminhos irredutíveis com relação a $\mathcal{R}^{\prime}$. Pelo Teorema 11 . $k Q / I=k Q / I^{\prime} \operatorname{com} I^{\prime}=\left\langle s-f_{s}^{\prime}\right\rangle_{s \in S}$, mas $I^{\prime} \subseteq I$ pois $s-f_{s} \in I$ e $f_{s}-f_{s}^{\prime} \in I$ para todo $s \in S$. Portanto $I=\left\langle s-f_{s}^{\prime}\right\rangle_{s \in S}$. Toda redução com relação a $\mathcal{R}^{\prime}$ é uma composição de reduções relativas 
a $\mathcal{R}$, logo todo caminho é de redução única com relação a $\mathcal{R}^{\prime}$. Como $f_{s}^{\prime}$ é irredutível com relação a $\mathcal{R}$ e $\mathcal{B}=\mathcal{B}^{\prime}$ então $f_{s}^{\prime}$ é irredutível com relação a $\mathcal{R}^{\prime}$.

\subsubsection{Construção de um sistema de redução que satisfaz a condição do Dia- mante}

Lema 14. Seja $r=\left(r_{n}, \ldots, r_{1}\right)$ uma redução com $r_{k}=r_{a_{k}, \rho_{k}, c_{k}}, \rho_{k}=\left(s_{k}, f_{k}\right)$ para todo $1 \leq k \leq n$. Então $x-r(x) \in\left\langle\left\{s_{k}-f_{k} \mid 1 \leq k \leq n\right\}\right\rangle$.

Teorema 12. Seja I um ideal bilateral de $k Q$ gerado por um conjunto enumerável $X$. Existe um sistema de redução $\mathcal{R}^{\prime}$ tal que

(1) I é o ideal bilateral gerado pelo conjunto $\{s-f\}_{(s, f) \in \mathcal{R}^{\prime}}$,

(2') Todo caminho é de redução finita, e

(3) Para todo $(s, f) \in \mathcal{R}^{\prime}, f$ é irredutivel.

Demonstração. Fixemos uma boa ordem sobre $Q_{0} \cup Q_{1}$, e uma função $\omega: Q_{1} \rightarrow \mathbb{N} \backslash\{0\}$. Considere a ordem total $\leq_{\omega}$ peso-lexicográfica sobre $Q_{\geq 0}$. Dado $x \in X$, podemos expressar $x$ da forma $x=s_{x}-f_{x}$ de tal maneira que $s_{x}$ seja mônico e $s_{x}>_{\omega} f_{x}$. Defina $\mathcal{R}_{X}:=\left\{\left(s_{x}, f_{x}\right)\right\}_{x \in X}$. O sistema $\mathcal{R}_{X}$ satisfaz as condições (1) e $\left(2^{\prime}\right)$. Se $\mathcal{R}_{X}$ satisfaz (3) o resultado está mostrado. Caso contrário, defina

$$
\mathcal{R}^{\prime}:=\left\{\left(s_{x}, r_{x}\left(f_{x}\right)\right) \mid r_{x} \text { é uma redução tal que } r_{x}\left(f_{x}\right) \text { é irredutível, } x \in X\right\}
$$

$\mathcal{R}^{\prime}$ está bem definido pois todo caminho é de redução finita com relação a $\mathcal{R}_{X} \cdot \mathcal{R}^{\prime}$ satisfaz as condições $\left(2^{\prime}\right)$ e (3), vamos mostrar que satisfaz (1). Note que $\pi\left(r_{x}\left(f_{x}\right)\right)=\pi\left(f_{x}\right)=\pi\left(s_{x}\right)$, logo $\pi\left(s_{x}-r_{x}\left(f_{x}\right)\right)=0 \mathrm{e}$

$$
\left\langle s_{x}-r_{x}\left(f_{x}\right)\right\rangle_{x \in X} \subseteq\left\langle s_{x}-f_{x}\right\rangle_{x \in X}=I
$$

Por conveniência vamos reescrever $\mathcal{R}_{X}$ da forma $\left\{\left(s_{i}, f_{i}\right)\right\}_{i \in \mathbb{N}}$ de tal maneira que $s_{i} \leq_{\omega} s_{i+1}$ para todo $i \in \mathbb{N}$. Vamos mostrar por indução que $s_{i}-f_{i} \in\left\langle s_{x}-r_{x}\left(f_{x}\right)\right\rangle_{x \in X}$ para todo $i \in \mathbb{N}$. Como $s_{1} \leq_{\omega} s_{i}$ para todo $i \in \mathbb{N}$ e todo termo que aparece em $f_{1}$ é estritamente menor que $s_{1}$, então $f_{1}$ é irredutível. Logo, $r_{1}\left(f_{1}\right)=f_{1}$. Seja $i>1$ e suponha $s_{j}-f_{j} \in\left\langle s_{x}-r_{x}\left(f_{x}\right)\right\rangle_{x \in X}$ para todo $j \leq i$. O elemento $f_{i+1}$ só pode ter termos divisíveis por $s_{j}$ com $j \leq i$. Pelo Lema (14) $r_{i+1}\left(f_{i+1}\right)-f_{i+1} \in\left\langle s_{j}-f_{j}\right\rangle_{j=1}^{i}$, e $\left\langle s_{j}-f_{j}\right\rangle_{j=1}^{i} \subseteq\left\langle s_{x}-r_{x}\left(f_{x}\right)\right\rangle_{x \in X}$ pela hipótese de indução. Logo, $r_{i+1}\left(f_{i+1}\right)-f_{i+1} \in\left\langle s_{x}-r_{x}\left(f_{x}\right)\right\rangle_{x \in X}$, o que implica $s_{i+1}-f_{i+1} \in\left\langle s_{x}-r_{x}\left(f_{x}\right)\right\rangle_{x \in X}$.

Exemplo 11. Vamos ver que nem todo sistema de redução que satisfaz as condições do Lema 12. pode ser obtido pelo método usado na demonstração do mesmo Lema. Seja

$$
A=k\langle x, y, z\rangle /\left(x^{3}+y^{3}+z^{3}-x y z\right),
$$

e $\mathcal{R}=\left\{\left(x y z, x^{3}+y^{3}+z^{3}\right)\right\}$. Claramente $\mathcal{R}$ satisfaz as condições (1) e (3). Podemos expressar de maneira única todo caminho (monômio) em A por $p=x_{1}^{a_{1}} x_{2}^{a_{2}} \cdots x_{n}^{a_{n}}$, com $x_{i} \in\{x, y, z\}, x_{i} \neq x_{i+1}$ para todo $1 \leq i \leq n-1$, e $a_{i} \in \mathbb{N} \backslash\{0\}$ para todo $i$. Vamos mostrar por indução sobre $n$ que todo caminho é de redução finita. Se $n=1, p$ é irredutivel. Vamos supor que todo caminho que é produto de potencias de $n$ variáveis ou menos é de redução finita, e seja $p=x_{1}^{a_{1}} x_{2}^{a_{2}} \cdots x_{n+1}^{a_{n+1}}$. Se existe uma redução básica tal que $r(p) \neq p$ é porque $p=x_{1}^{a_{1}} x_{2}^{a_{2}} \cdots x_{k}^{a_{k}} x y z x_{k+4}^{a_{k+4}} \cdots x_{n+1}^{a_{n+1}}$, e $r=r_{a, \rho, c}$ com $a=x_{1}^{a_{1}} x_{2}^{a_{2}} \cdots x_{k}^{a_{k}}, c=x_{k+4}^{a_{k+4}} \cdots x_{n+1}^{a_{n+1}}$ e $\rho=\left(x y z, x^{3}+y^{3}+z^{3}\right)$. Nesse caso

$$
r(p)=x_{1}^{a_{1}} x_{2}^{a_{2}} \cdots x_{k}^{a_{k}}\left(x^{3}+y^{3}+z^{3}\right) x_{k+4}^{a_{k+4}} \cdots x_{n+1}^{a_{n+1}} .
$$

Note que cada um dos termos em $r(p)$ satisfaz a hipótese de indução. Pela hipótese de indução cada um dos termos é de redução finita, portanto $r(p)$ é de redução finita. Isto implica que $p$ é de redução 
finita.

$O$ sistema $\mathcal{R}$ não pode ser obtido pelo método anterior, pois dada qualquer ordem $\leq$ sobre $\{x, y, z\}$ e qualquer função peso $\omega$ temos $\omega(x y z)=\omega(x)+\omega(y)+\omega(z) \leq \omega\left(u^{3}\right)$, com $u=\max _{\leq_{\omega}}\{x, y, z\}$.

Ou seja não é possível via uma ordem admissível obter o sistema acima. No entanto, por exemplo para construir resoluções projetivas, este sistema é muito bom. Esse exemplo mostra uma vantagem de usar os métodos de Chouhy e Solotar sobre os métodos de Green de ordens admissíveis. Em alguns casos é muito interessante considerar sistemas que satisfazem as condições do lema do diamante, mesmo que eles não sejam obtidos por ordens admissíveis. No entanto existe toda uma teoria desenvolvida usando ordens admissíveis, isso justifica o seu estudo.

Definição 30. Seja $\mathcal{R}$ um sistema de redução:

- Uma ambiguidade de inclusão é uma 5-upla $\left(\rho_{1}, \rho_{2}, a, b, c\right)$ com $\rho_{1}, \rho_{2} \in \mathcal{R}, a, b, c \in Q_{\geq 0}$, tal que $\rho_{1}=\left(a b c, f_{1}\right)$ e $\rho_{2}=\left(b, f_{2}\right)$ para $f_{1}, f_{2} \in k Q$.

Se existem reduções $r=\left(r_{n}, \ldots, r_{1}\right), t=\left(t_{m}, \ldots, t_{1}\right)$ tais que $r_{1}=r_{1, \rho_{1}, 1}, t_{1}=r_{a, \rho_{2}, c} e$ $r(a b c)=t(a b c)$, dizemos que $\left(\rho_{1}, \rho_{2}, a, b, c\right)$ é resolvivel.

- Uma ambiguidade de sobreposição é uma 5-upla $\left(\rho_{1}, \rho_{2}, a, b, c\right)$ com $\rho_{1}, \rho_{2} \in \mathcal{R}, a, b, c \in$ $Q_{\geq 0}$, tal que $\rho_{1}=\left(a b, f_{1}\right)$ e $\rho_{2}=\left(b c, f_{2}\right)$ para $f_{1}, f_{2} \in k Q$. Dizemos que uma ambiguidade de sobreposição $\left(\rho_{1}, \rho_{2}, a, b, c\right)$ é minimal se não existem ambiguidades de sobreposição $\left(\rho_{1}^{\prime}, \rho_{2}^{\prime}, x, y, z\right)$ com xyz divisor próprio de abc.

Se existem reduções $r=\left(r_{n}, \ldots, r_{1}\right), t=\left(t_{m}, \ldots, t_{1}\right)$ tais que $r_{1}=r_{1, \rho_{1}, c}, t_{1}=r_{a, \rho_{2}, 1} e$ $r(a b c)=t(a b c)$, dizemos que $\left(\rho_{1}, \rho_{2}, a, b, c\right)$ é resolvivel.

- Uma ambiguidade é uma ambiguidade de inclusão ou uma ambiguidade de sobreposição.

Proposição 14. Seja $\mathcal{R}$ um sistema de redução que satisfaz as condições (1), (2') e (3). $\mathcal{R}$ satisfaz a condição do Diamante se e somente se todas as ambiguidades são resolvíveis.

Exemplo 12. Seja $A=k\langle x, y\rangle / I$, com $I=\left\langle x^{2}, y^{2}, y x-\xi x y\right\rangle$. Fixamos a ordem sobre as flechas $x<$ y e a função peso dada por $\omega(x)=\omega(y)=1$. Seguindo o processo dado no Teorema(12) e o conjunto de geradores $X=\left\{x^{2}, y^{2}, y x-\xi x y\right\}$ obtemos o sistema de redução $\mathcal{R}_{X}=\left\{\left(x^{2}, 0\right),\left(y^{2}, 0\right),(y x, \xi x y)\right\}$. Este sistema satisfaz as condições (1), $\left(2^{\prime}\right)$ e (3). Para verificar se as ambiguidades são resolvíveis vamos notar os elementos do sistema por $\rho_{1}=\left(x^{2}, 0\right), \rho_{2}=(y x, \xi x y)$ e $\rho_{3}=\left(y^{2}, 0\right)$.

Note que não existem ambiguidades de inclusão e que o conjunto de ambiguidades minimais de sobreposição é

$$
\left\{\left(\rho_{1}, \rho_{1}, x, x, x\right),\left(\rho_{2}, \rho_{1}, y, x, x\right),\left(\rho_{3}, \rho_{2}, y, y, x\right),\left(\rho_{3}, \rho_{3}, y, y, y\right)\right\}
$$

$A$ ambiguidade $\left(\rho_{1}, \rho_{1}, x, x, x\right)$ é resolvivel pois $r=r_{1, \rho_{1}, x}$ et $t=r_{x, \rho_{1}, 1}$ satisfazem $r\left(x^{3}\right)=t\left(x^{3}\right)=0$. Do mesmo modo podemos mostrar que $\left(\rho_{3}, \rho_{3}, y, y, y\right)$ é resolvível. Para mostrar que a ambiguidade $\left(\rho_{3}, \rho_{2}, y, y, x\right)$ é resolvivel consideramos as reduções $r=r_{1, \rho_{3}, x}$ e $t=\left(r_{x, \rho_{3}, 1}, r_{1, \rho_{2}, y}, r_{y, \rho_{2}, 1}\right)$. Nesse caso $r\left(y^{2} x\right)=t\left(y^{2} x\right)=0$. As reduções $r=\left(r_{1, \rho_{1}, y}, r_{x, \rho_{2}, 1}, r_{1, \rho_{2}, x}\right)$ e $t=r_{y, \rho_{1}, 1}$ fazem a ambiguidade $\left(\rho_{2}, \rho_{1}, y, x, x\right)$ resolvível. Portanto, o sistema de redução $\mathcal{R}_{X}$ satisfaz a condição do Diamante para I.

Exemplo 13. No Exemplo (11) o sistema $\mathcal{R}=\left\{\left(x y z, x^{3}+y^{3}+z^{3}\right)\right\}$ não tem ambiguidades, logo $\mathcal{R}$ satisfaz a condição do diamante para I. Vamos construir outro sistema de redução usando o processo do Teorema (12). Considere a ordem $x<y<z$ e a função peso dada por $\omega(x)=\omega(y)=\omega(z)=1$. Seja $X=x^{3}+y^{3}+z^{3}-x y z$. Com isto o sistema de redução é $\mathcal{R}_{X}=\left\{\left(z^{3}, x y z-x^{3}-y^{3}\right)\right\}$ e satisfaz as condições (1), $\left(2^{\prime}\right)$ e (3). A única ambiguidade minimal neste caso é $\left(\rho, \rho, z, z^{2}, z\right)$ onde $\rho=\left(z^{3}, x y z-x^{3}-y^{3}\right)$. Esta ambiguidade não resolvível pois $r_{z, \rho, 1}\left(z^{4}\right)=z x y z-z x^{3}-z y^{3} e$ $r_{1, \rho, z}\left(z^{4}\right)=x y z^{2}-x^{3} z-y^{3} z$ são irredutiveis e distintos. Portanto $\mathcal{R}_{X}$ não satisfaz a condição do Diamante para $I$. 
Se temos um sistema de redução $\mathcal{R}$ que satisfaz as condições (1), (2') e (3) mas tem ambiguidades não resolvíveis podemos adicionar elementos ao sistema de tal forma que o novo sistema tenha ditas ambiguidades resolvíveis. De fato, se $\rho=\left(\rho_{1}, \rho_{2}, a, b, c\right)$ é uma ambiguidade não resolvível, escolha duas reduções $r=\left(r_{n}, \ldots, r_{1}\right)$ e $t=\left(t_{m}, \ldots, t_{1}\right)$ tais que $r(a b c)$ e $t(a b c)$ são irredutíveis distintos com $r_{1}$ e $t_{1}$ como na Definição(30). Podemos escrever $r(a b c)-t(a b c)$ da forma $s-f \operatorname{com} f<_{\omega} s$ e seja $f^{\prime}$ irredutivel tal que $r(f)=f^{\prime}$ para alguma redução $r$. O sistema $\mathcal{R}^{\prime}:=\mathcal{R} \cup\left\{\left(s, f^{\prime}\right)\right\}$ satisfaz as condições $(1),\left(2^{\prime}\right)$ e (3) e $\rho$ é resolvível com relação a este novo sistema. Ao adicionar o novo elemento ao sistema poderiam aparecer novas ambiguidades não resolvíveis e nesse caso repetimos o passo anterior tantas vezes quanto forem necessárias (este processo poderia ser infinito) até não ter ambiguidades não resolvíveis, obtendo assim um sistema de redução que satisfaz a condição do Diamante para $I$.

Exemplo 14. Sejam

$$
A=k\langle x, y, z\rangle /\left(x^{3}+y^{3}+z^{3}-x y z\right),
$$

e $\mathcal{R}=\left\{\left(z^{3}, x y z-x^{3}-y^{3}\right)\right\}$. No Exemplo(13) vimos que $\left(\rho, \rho, z, z^{2}, z\right)$ com $\rho=\left(z^{3}, x y z-x^{3}-y^{3}\right)$ é uma ambiguidade não resolvível pois $r_{z, \rho, 1}\left(z^{4}\right)=z x y z-z x^{3}-z y^{3}$ e $r_{1, \rho, z}\left(z^{4}\right)=x y z^{2}-x^{3} z-y^{3} z$ são irredutiveis distintos. A diferença destes dois irredutiveis é $x y z^{2}-x^{3} z-y^{3} z-z x y z+z x^{3}+z y^{3}$. Defina $\mathcal{R}_{1}=\left\{\rho, \rho_{1}\right\}$, onde

$$
\rho_{1}=\left(x y z^{2}, x^{3} z+y^{3} z+z x y z-z x^{3}-z y^{3}\right) .
$$

$A$ ambiguidade $\left(\rho, \rho, z, z^{2}, z\right)$ agora é resolvivel. De fato, se $r=\left(r_{1, \rho_{1}, 1}, r_{1, \rho, z}\right)$ e $t=r_{z, \rho .1}$, então $r\left(z^{4}\right)=t\left(z^{4}\right)=z x y z-z x^{3}-z y^{3}$. O novo conjunto de ambiguidades é $\left\{\left(\rho, \rho, z, z^{2}, z\right),\left(\rho_{1}, \rho, x y, z^{2}, z\right)\right\}$. Esta nova ambiguidade não é resolvível. Temos que $r_{z, \rho_{1}, 1} \circ r_{1, \rho_{1}, z}\left(x y z^{3}\right)=x^{3} z^{2}+y^{3} z^{2}+z^{2} x y z-$ $z^{2} x^{3}-z^{2} y^{3}$ e $r_{x y, \rho, 1}\left(x y z^{3}\right)=x y x y z-x y x^{3}-x y^{4}$ são dois irredutiveis distintos. Logo, o novo sistema é $\mathcal{R}_{2}:=\left\{\rho, \rho_{1}, \rho_{2}\right\}$, onde

$$
\rho_{2}=\left(y^{3} z^{2},-x^{3} z^{2}-z^{2} x y z+z^{2} x^{3}+z^{2} y^{3}+x y x y z-x y x^{3}-x y^{4}\right) .
$$

A ambiguidade $\left(\rho_{1}, \rho, x y, z^{2}, z\right)$ é resolvível com relação a $\mathcal{R}_{2}$. Ao adicionar $\rho_{2}$ ao sistema aparece a ambiguidade $\left(\rho_{2}, \rho, y^{3}, z^{2}, z\right)$ a qual resulta ser resolvivel. Portanto, o sistema $\mathcal{R}_{2}$ satisfaz a condição do Diamante para $I$.

Corolário 5. Seja $\leq$ uma ordem sobre $Q_{0} \cup Q_{1}$, $\omega$ uma função peso e I um ideal bilateral de $k Q$. Se $X$ é um conjunto de relações que geram I e $\mathcal{R}_{X}$ satisfaz as condições (1), (2') e (3)e toda ambiguidade é resolvivel, então $\left\{(s, f) \in \mathcal{R}_{X} \mid s \notin \operatorname{Inc}\left(\mathcal{R}_{X}\right)\right\}$ é um sistema de redução que satisfaz a condição do Diamante para I, não tem ambiguidades de inclusão e

$$
\mathcal{R}_{\leq, \omega}=\left\{(s, f) \in \mathcal{R}_{X} \mid s \notin \operatorname{Inc}\left(\mathcal{R}_{X}\right)\right\}
$$

Além disso, $p \in \operatorname{tip}(I)$ se e somente se existe $q \in \operatorname{tip}(X)$ tal que $q$ divide $p$.

Nota 7. Dado I um ideal bilateral de $k Q$ e $\mathcal{R}$ um sistema de redução que satisfaz a condição do Diamante para I, podemos assumir $S \subseteq Q_{\geq 2}$. Este fato e o Corolário anterior são demonstrados em [CS15] e são de muita utilidade para o nosso objetivo.

\subsection{Ambiguidades}

Seja $I$ um ideal bilateral de $k Q$ e $\mathcal{R}$ um sistema de redução que satisfaz a condição do Diamante para $I$. Podemos assumir que $\mathcal{R}$ não tem ambiguidades de inclusão e $S \subseteq Q_{\geq 2}$.

Definição 31. Seja $n \geq 2$ e $p \in Q_{\geq 0}$,

1. O caminho p é uma n-ambiguidade a esquerda se existem $u_{0} \in Q_{1}$ e $u_{1}, \ldots, u_{n-1}$ caminhos irredutiveis tais que 
(a) $p=u_{0} u_{1} \cdots u_{n-1}$,

(b) Para todo $i$, o caminho $u_{i} u_{i+1}$ é redutivel mas $u_{i} d$ é irredutivel para todo divisor próprio a esquerda de $u_{i+1}$.

2. O caminho $p$ é uma $n$-ambiguidade a direita se existem $v_{0} \in Q_{1}$ e $v_{1}, \ldots, v_{n-1}$ caminhos irredutiveis tais que

(a) $p=v_{n-1} \cdots v_{1} v_{0}$,

(b) Para todo $i$, o caminho $v_{i+1} v_{i}$ é redutivel mas $d v_{i}$ é irredutível para todo divisor próprio a direita de $v_{i+1}$.

Definimos $\mathcal{A}_{0}:=Q_{0}, \mathcal{A}_{1}:=Q_{1}, \mathcal{A}_{2}:=S$ e para $n \geq 2 \mathcal{A}_{n}$ e $\mathcal{A}_{n}^{\prime}$ são o conjunto de $n$-ambiguidades a esquerda e a direita respectivamente.

As ambiguidades para caminhos foram introduzidas por Green e Zacharia, para o estudo da álgebra de extensões de uma álgebra monomial e o conjunto das n-ambiguidades foram chamados por eles de $A P(n)\left[\mathrm{GHZ}^{+} 85\right]$.

A seguir apresentamos alguns resultados importantes envolvendo os conjuntos das $n$-ambiguidades e que não demonstraremos aqui. As demonstrações podem ser encontradas em [Bar97] e [CS15].

Proposição 15. Sejam $n, m \in \mathbb{N}$ e $p \in Q_{\geq 1}$. Se $u_{0}, \widehat{u}_{0} \in Q_{1}, u_{1}, \ldots, u_{n-1}, \widehat{u}_{1}, \ldots, \widehat{u}_{n-1}$ são caminhos em $Q$ tais que $u_{0}, \ldots, u_{n-1}$ e $\widehat{u}_{0}, \ldots, \widehat{u}_{n-1}$ satisfazem as condições $(1 a)$ e (1b) da Definição(31) para $p$, então $n=m$ e $u_{i}=\widehat{u}_{i}$ para $0 \leq i \leq n-1$.

Corolário 6. Dado $n, m \geq 0, \mathcal{A}_{n} \cap \mathcal{A}_{m}=\emptyset$ se $n \neq m$.

Proposição 16. Suponha $S \subseteq Q_{2}$. Para todo $n \geq 1$, podemos definir o conjunto das $n$-ambiguidades por

$$
\mathcal{A}_{n}=\left\{\alpha_{0} \cdots \alpha_{n-1} \in Q_{n} \mid \alpha_{i} \in Q_{1} \text { para todo } i \text { e } \alpha_{i-1} \alpha_{i} \in S\right\} .
$$

Esta proposição fornece uma ferramenta que facilita o calculo das $n$-ambiguidades em casos específicos como podemos ver nos seguintes exemplos.

Exemplo 15. Seja $A=k\langle x, y\rangle / I$, com $I=\left\langle x^{2}, y^{2}, y x-\xi x y\right\rangle$ como no Exemplo 12. Já mostramos que o sistema $\mathcal{R}=\left\{\left(x^{2}, 0\right),\left(y^{2}, 0\right),(y x, \xi x y)\right\} e ́$ um sistema de redução que satisfaz a condição do Diamante para I. Este sistema não tem ambiguidades de inclusão e $S=\left\{x^{2}, y^{2}, y x\right\} \subseteq Q_{2}$. Pela Proposição 16 o conjunto $\mathcal{A}_{n}$ coincide com o conjunto de caminhos de comprimento $n$ que não são divisiveis por $x y$, ou seja

$$
\mathcal{A}_{n}=\left\{y^{s} x^{t} \mid s+t=n\right\}
$$

\subsection{1 Álgebras monomiais}

Seja $A=k Q / I$ com $I$ um ideal admissível gerado por um número finito de caminhos em $Q$. Neste caso dizemos que $A$ é uma álgebra monomial. Vamos fixar uma ordem $<$ sobre $Q_{0} \cup Q_{1}$ e a partir desta ordem $<$ a ordem comprimento-lexicográfica sobre o conjunto de todos os caminhos lendo da origem ao término de cada caminho. Esta ordem admissível permite construir um conjunto finito de geradores do $I$ notado por $\operatorname{Minsharp}_{<}(I)$ e tal que se $p \in \operatorname{Minsharp} p_{<}(I)$ então nenhum subdivisor próprio de $p$ pertence a $\operatorname{Minsharp}_{<}(I)$ [FFG93]. Defina $\mathcal{R}:=\left\{(s, 0) \mid s \in \operatorname{Minsharp}_{<}(I)\right\}$ e note que $\mathcal{R}$ é um sistema de redução que satisfaz a condição do Diamante para $I$. Além disso, $S \subseteq Q_{\geq 2}$ pelo fato de $I$ ser um ideal admissível.

Em [Bar97] Bardzell constroi resoluções projetivas a partir dos elementos dos conjuntos notados $A P(n)$, os quais vamos definir usando os elementos do $\operatorname{Minsharp}_{<}(I)$ e mostrar que no caso 
monomial coincidem com os conjuntos das $n$-ambiguidades.

Seja $p_{i} \in \operatorname{Minsharp}_{<}(I)=\left\{p_{1}, p_{2}, \ldots, p_{m}\right\}, \Upsilon$ um caminho dirigido tal que $p_{i}$ divide a $\Upsilon$ e seja Minsharp $p_{<}^{\Upsilon}(I)$ o conjunto dos elementos em $\operatorname{Minsharp}_{<}(I)$ que dividem $\Upsilon$. Escolha $r_{2} \in$ Minsharp $p_{<}^{\Upsilon}(I)$ tal que $o\left(p_{i}\right) \lesssim o\left(r_{2}\right) \lesssim t\left(p_{i}\right)$ sobre $\Upsilon$, com $o\left(r_{2}\right)$ minimal com relação a $\lesssim$ (caso ele exista). Suponha que já escolhemos $r_{1}=p_{i}, r_{2}, \ldots, r_{j}$ e seja

$$
L_{j+1}=\left\{r \in \operatorname{Minsharp}{ }_{<}^{\Upsilon}(I) \mid t\left(r_{j-1}\right) \lesssim o(r) \lesssim t\left(r_{j}\right)\right\} .
$$

Se $L_{j+1} \neq \emptyset$ escolha $r_{j+1} \in L_{j+1}$ com $o\left(r_{j+1}\right)$ minimal com relação a $\lesssim$.

Para todo $n \geq 2$, a sequência $\left(r_{1}, r_{2}, \ldots, r_{n}\right)$ construída define o caminho $p_{i}^{n}$ com origem $o\left(r_{1}\right)$ e término $t\left(r_{n}\right)$. Tomando todos os caminhos $\Upsilon$ que $p_{i}$ divide e os $p_{i}^{n}$ correspondentes formamos o conjunto $A P_{i}(n)$. Finalmente, fazemos o processo anterior para cada $p_{i} \in \operatorname{Minsharp}_{<}(I)$, obtendo o conjunto

$$
A P(n):=\bigcup_{i=1}^{m} A P_{i}(n)
$$

O seguinte gráfico permite ver uma correspondência natural entre $A P(n)$ e $\mathcal{A}_{n}$ para todo $n \geq 2$.

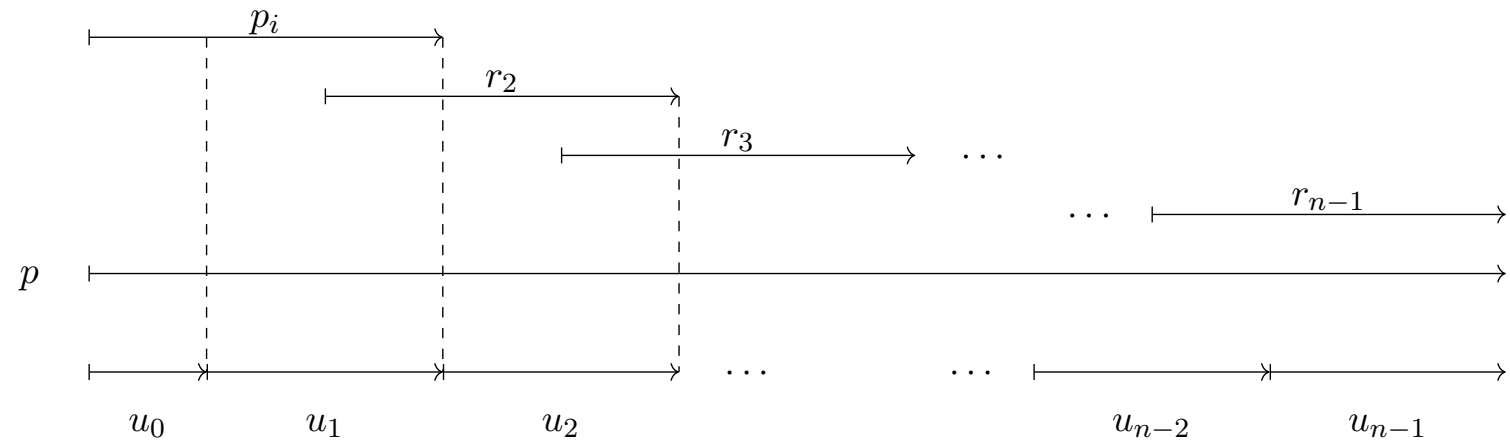

Definimos por $A P(0)$ o conjunto dos vertices e por $A P(1)$ o conjunto das flechas. Note que $A P(2)$ coincide com o conjunto de geradores $\operatorname{Minsharp}_{<}(I)$.

Exemplo 16. Seja $A=k Q / I$ com $Q$ o seguinte carcás

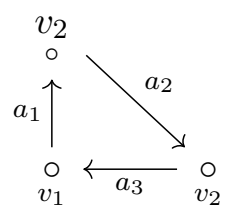

e $I=\left\langle a_{1} a_{2}, a_{2} a_{3}, a_{3} a_{1}\right\rangle$. Neste caso $A P(0)=\left\{v_{1}, v_{2}, v_{3}\right\}$ e AP(1) $=\left\{a_{1}, a_{2}, a_{3}\right\}$. Os elementos geradores de $I$ já fornecem os elementos de $A P(2)$ pois não se dividem entre eles, logo $A P(2)=$ $\left\{a_{1} a_{2}, a_{2} a_{3}, a_{3} a_{1}\right\}$. Pela Proposição 16 AP $(n)$ é o conjunto de todos os caminhos (os três caminhos que começam em cada um dos vértices) de comprimento $n$, para todo $n \geq 2$.

\subsection{Resolução projetiva}

Dada uma álgebra $A=k Q / I$ e um sistema de redução $\mathcal{R}$ que satisfaz a condição do Diamante para $I$ existe uma álgebra monomial associada a $A$ dada por $A_{S}:=k Q /\langle S\rangle$, a qual tem como $k$-base 
$\pi^{\prime}(\mathcal{B})$, onde $\pi^{\prime}$ é a projeção canônica $\pi^{\prime}: k Q \rightarrow A_{S}$.

Podemos definir aplicações $k$-lineares $i: A \rightarrow k Q$ e $i^{\prime}: A_{S} \rightarrow k Q$ tais que $i(\pi(b))=i^{\prime}\left(\pi^{\prime}(b)\right)=b$ para todo $b \in \mathcal{B}$.

Note que o $k Q$-bimódulo $k Q \otimes_{E} k \mathcal{A}_{n} \otimes_{E} k Q$ é um $k$-espaço vetorial com base $\{a \otimes p \otimes c \mid a, c \in$ $Q_{\geq 0}, p \in \mathcal{A}_{n}, a p c \neq 0$ em $\left.k Q\right\}$. Considere a seguinte sequência de $k Q$ bimódulos e aplicações

$$
\ldots \stackrel{f_{3}}{\longrightarrow} k Q \otimes_{E} k \mathcal{A}_{2} \otimes_{E} k Q \stackrel{f_{2}}{\longrightarrow} k Q \otimes_{E} k \mathcal{A}_{1} \otimes_{E} k Q \stackrel{f_{1}}{\longrightarrow} k Q \otimes_{E} k Q \stackrel{f_{0}}{\longrightarrow} k Q \rightarrow 0,
$$

onde

(1) $f_{0}(a \otimes b)=a b$,

(2) Se $n$ é impar, $f_{n}$ é o homomorfismo de $k Q$-bimódulos dado por

$$
f_{n}(1 \otimes q \otimes 1)=v_{n-1} \otimes v_{n-2} \cdots v_{0} \otimes 1-1 \otimes u_{0} \cdots u_{n-2} \otimes u_{n-1},
$$

para todo $q \in \mathcal{A}_{n}$, onde $q=u_{0} \cdots u_{n-1}=v_{n-1} \cdots v_{0}$ são as fatorações a esquerda e direita de $q$ como $n$-ambiguidade respectivamente.

(3) Se $n$ é par, $f_{n}$ é o homomorfismo de $k Q$-bimódulos dado por

$$
f_{n}(1 \otimes q \otimes 1)=\sum_{\substack{a p c=q \\ p \in \mathcal{A}_{n-1}}} a \otimes p \otimes c,
$$

para todo $q \in \mathcal{A}_{n}$.

Notação: Para $n \geq 0$ fixamos a seguinte notação

$$
\begin{array}{cl}
\pi_{n}:=\pi \otimes i d_{k \mathcal{A}_{n}} \otimes \pi, & \pi_{n}^{\prime}:=\pi^{\prime} \otimes i d_{k \mathcal{A}_{n}} \otimes \pi^{\prime} \\
i_{n}:=i \otimes i d_{k \mathcal{A}_{n}} \otimes i, & i_{n}^{\prime}:=i^{\prime} \otimes i d_{k \mathcal{A}_{n}} \otimes i^{\prime}, \\
\beta_{n}:=i_{n} \circ \pi_{n}, & \beta_{n}^{\prime}:=i_{n}^{\prime} \circ \pi_{n}^{\prime} .
\end{array}
$$

Estes homomorfismos $f_{n}$ induzem homomorfismos de $A$-bimódulos e $A_{S^{-}}$-bimódulos dados por $\delta_{n}:=$ $\pi_{n-1} \circ f_{n} \circ i_{n}$ e $\delta_{n}^{\prime}:=\pi_{n-1}^{\prime} \circ f_{n} \circ i_{n}^{\prime}$ respectivamente. Temos o seguinte diagrama comutativo onde a primeira linha é a resolução projetiva mencionada e a segunda linha é a sequência de $A$ bimódulos junto com as aplicações induzidas $\delta_{n}$.

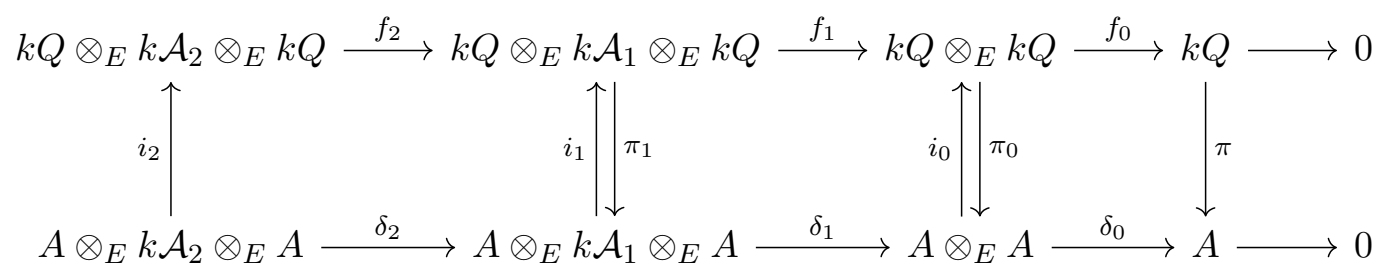

Analogamente, obtemos o complexo de $A_{S}$-bimódulos

$$
\cdots \stackrel{\delta_{3}^{\prime}}{\longrightarrow} A_{S} \otimes_{E} k \mathcal{A}_{2} \otimes_{E} A_{S} \stackrel{\delta_{2}^{\prime}}{\longrightarrow} A_{S} \otimes_{E} k \mathcal{A}_{1} \otimes_{E} A_{S} \stackrel{\delta_{1}^{\prime}}{\longrightarrow} A_{S} \otimes_{E} A_{S} \stackrel{\delta_{0}^{\prime}}{\longrightarrow} A_{S} \longrightarrow 0,
$$

Este complexo é uma resolução, pois de fato coincide com a resolução projetiva dada por Bardzell em [Bar97]. Para obter uma resolução projetiva de $A$ são necessárias mais ferramentas além do complexo de $A$-bimódulos induzido pelos $f_{n}$. 
Exemplo 17. Em [Bar97] Bardzell calcula uma resolução projetiva para a álgebra do Exemplo 16 e a partir desta resolução apresenta os grupos de cohomologia. Vamos mostrar o calculo dos grupos de cohomologia usando a notação de Bardzell, mas lembrando que coincide com a resolução projetiva anterior, pois neste caso $A_{S}=A$.

Usando os conjuntos das n-ambiguidades de A temos a resolução projetiva para A:

$$
\cdots \rightarrow P_{4} \stackrel{\phi_{4}}{\longrightarrow} P_{3} \stackrel{\phi_{3}}{\longrightarrow} P_{2} \stackrel{\phi_{2}}{\longrightarrow} P_{1} \stackrel{\phi_{1}}{\longrightarrow} P_{0} \rightarrow A \rightarrow 0
$$

onde

$$
\begin{aligned}
& P_{0}=\left(A v_{1} \otimes v_{1} A\right) \oplus\left(A v_{2} \otimes v_{2} A\right) \oplus\left(A v_{3} \otimes v_{3} A\right)=P_{3}=\cdots \\
& P_{1}=\left(A v_{1} \otimes v_{2} A\right) \oplus\left(A v_{2} \otimes v_{3} A\right) \oplus\left(A v_{3} \otimes v_{1} A\right)=P_{4}=\cdots \\
& P_{2}=\left(A v_{1} \otimes v_{3} A\right) \oplus\left(A v_{2} \otimes v_{1} A\right) \oplus\left(A v_{3} \otimes v_{2} A\right)=P_{5}=\cdots
\end{aligned}
$$

Aplicando o functor $\mathrm{Hom}_{A^{e}}(\cdot$, A) e usando o isomorfismo

$$
\operatorname{Hom}_{A^{e}}\left(\coprod_{A P(n)} A o\left(p^{n}\right) \otimes t\left(p^{n}\right) A, A\right) \cong \coprod_{A P(n)} o\left(p^{n}\right) A t\left(p^{n}\right)
$$

calcular os grupos de cohomologia de A se reduz a calcular a cohomologia do seguinte complexo

$$
0 \rightarrow P_{0}^{*} \stackrel{\phi_{1}^{*}}{\rightarrow} P_{1}^{*} \stackrel{0}{\rightarrow} P_{2}^{*} \stackrel{0}{\rightarrow} P_{3}^{*} \stackrel{\phi_{4}^{*}}{\rightarrow} P_{4}^{*} \rightarrow \cdots
$$

onde

$$
\begin{aligned}
& P_{0}^{*}=v_{1} A v_{1} \oplus v_{2} A v_{2} \oplus v_{3} A v_{3}=P_{3}^{*}=\cdots \\
& P_{1}^{*}=v_{1} A v_{2} \oplus v_{2} A v_{3} \oplus v_{3} A v_{1}=P_{4}^{*}=\cdots \\
& P_{2}^{*}=v_{1} A v_{3} \oplus v_{2} A v_{1} \oplus v_{3} A v_{2}=0=P_{5}^{*}=\cdots
\end{aligned}
$$

Pela periodicidade de (17) basta calcular $H H^{i}(A)$ para $i=0,1,2,3,4,5$. Trivialmente $H H^{2}(A)=$ $H H^{5}(A)=0$.

Como $\phi_{1}(o(a) \otimes t(a))=a \otimes t(a)-o(a) \otimes$ a para toda flecha a então

$$
\begin{aligned}
\phi_{1}^{*}\left(v_{1}, 0,0\right) & =\left(a_{1} v_{1} t\left(a_{1}\right)-o\left(a_{1}\right) v_{1} a_{1}, a_{2} v_{1} t\left(a_{2}\right)-o\left(a_{2}\right) v_{1} a_{2}, a_{3} v_{1} t\left(a_{3}\right)-o\left(a_{3}\right) v_{1} a_{3}\right) \\
& =\left(-a_{1}, 0, a_{3}\right) .
\end{aligned}
$$

Analogamente $\phi_{1}^{*}\left(0, v_{2}, 0\right)=\left(a_{1},-a_{2}, 0\right)$ e $\phi_{1}^{*}\left(0,0, v_{3}\right)=\left(0, a_{2},-a_{3}\right)$.

Se $\alpha=\left(k_{1} v_{1}, k_{2} v_{2}, k_{3} v_{3}\right)$ então $\phi_{1}^{*}(\alpha)=\left(k_{2}-k_{1}\right)\left(a_{1}, 0,0\right)+\left(k_{3}-k_{2}\right)\left(0, a_{2}, 0\right)+\left(k_{1}-k_{3}\right)\left(0,0, a_{3}\right)$. Logo, se $\alpha \in \operatorname{Ker}\left(\phi_{1}^{*}\right)$ então $k_{1}=k_{2}=k_{3}$ e portanto $H H^{0}(A)=\left\{k\left(v_{1}, v_{2}, v_{3}\right) \mid k \in K\right\} \cong K$.

Para calcular $H H^{3}(A)$ basta calcular Ker $\left(\phi_{4}^{*}\right)$. Temos que $\phi_{4}(o(a) \otimes t(a))=a \otimes t(a)+o(a) \otimes a$ para toda flecha a, então $\phi_{4}^{*}\left(v_{1}, 0,0\right)=\left(a_{1}, 0, a_{3}\right), \phi_{4}^{*}\left(0, v_{2}, 0\right)=\left(a_{1}, a_{2}, 0\right)$ e $\phi_{4}^{*}\left(0,0, v_{3}\right)=\left(0, a_{2}, a_{3}\right)$, portanto $H H^{3}(A)=0$.

Como $\operatorname{dim} P_{0}^{*}=3$ e $\operatorname{dim}\left(\operatorname{ker}\left(\phi_{1}^{*}\right)\right)=2$ então $\operatorname{dim}\left(H H^{1}(A)\right)=1$ e $H H^{1}(A) \cong K$.

Finalmente $H H^{4}(A)=P_{1}^{*} / \operatorname{Im}\left(\phi_{4}^{*}\right) . S e j a\left(l_{1} a_{1}, l_{2} a_{2}, l_{3} a_{3}\right) \in P_{1}^{*}$, então 


$$
\phi_{4}^{*}\left(\frac{1}{2}\left(l_{1}-l_{2}+l_{3}\right) v_{1}, \frac{1}{2}\left(l_{2}+l_{1}-l_{3}\right) v_{2}, \frac{1}{2}\left(l_{2}-l_{1}+l_{3}\right) v_{3}\right)=\left(l_{1} a_{1}, l_{2} a_{2}, l_{3} a_{3}\right)
$$

Logo, $H H^{4}(A)=0$.

Em [Skö08] Sköldberg fornece uma contração de homotopia para a resolução projetiva de Bardzell. Neste caso mais geral vamos usar esta mesma ideia de contraçaõ de homotopia. Definimos o homomorfismo de $k Q-E$-bimódulos $S_{0}: k Q \rightarrow k Q \otimes_{E} k Q$ por $S_{0}(x)=x \otimes 1$ e para $n \geq 1$ definimos os homomorfismos de $k Q-E$-bimódulos $S_{n}: k Q \otimes_{E} k \mathcal{A}_{n-1} \otimes_{E} k Q \rightarrow k Q \otimes_{E} k \mathcal{A}_{n} \otimes_{E} k Q$ por:

$$
S_{n}(1 \otimes q \otimes b)=(-1)^{n} \sum_{\substack{a p c=q b \\ p \in \mathcal{A}_{n}}} a \otimes p \otimes c
$$

Os homomorfismos $S_{n}$ induzem homomorfismos de $A$ - bimódulos $s_{n}: A \otimes_{E} k \mathcal{A}_{n-1} \otimes_{E} A \rightarrow$ $A \otimes_{E} k \mathcal{A}_{n} \otimes_{E} A$ definidos por $s_{n}:=\pi_{n} \circ S_{n} \circ i_{n-1}$. Da mesma maneira temos homomorfismos de $A_{S}$-bimódulos $s_{n}^{\prime}: A_{S} \otimes_{E} k \mathcal{A}_{n-1} \otimes_{E} A_{S} \rightarrow A_{S} \otimes_{E} k \mathcal{A}_{n} \otimes_{E} A_{S}$ definidos por $s_{n}^{\prime}:=\pi_{n}^{\prime} \circ S_{n} \circ i_{n-1}^{\prime}$. A família de homomorfismos $\left\{s_{i}^{\prime}\right\}_{i \geq 0}$ coincide com a contração de homotopia dada por Sköldberg em [Skö08], logo as seguintes igualdades são satisfeitas

$$
s_{0}^{\prime} \circ \delta_{0}^{\prime}=i d_{A_{S} \otimes_{E} A_{S}} \quad, \quad s_{n}^{\prime} \circ \delta_{n}^{\prime}+\delta_{n-1}^{\prime} \circ s_{n-1}^{\prime}=i d_{A_{S} \otimes_{E} k \mathcal{A}_{n} \otimes_{E} A_{S}}
$$

para todo $n \geq 1$.

Definimos sobre o conjunto $k^{*} Q_{\geq 0}:=\left\{\lambda p: \lambda \in k^{*}, p \in Q_{\geq 0}\right\} \cup\{0\}$ a relação $\preceq$ como a menor relação reflexiva e transitiva tal que $\lambda p \preceq \mu q$ se existe uma redução $r$ tal que $r(\mu q)=\lambda p+x$, $\operatorname{com} p \notin \operatorname{supp}(x)$. Estabelecemos $0 \preceq \lambda p$ para todo $\lambda p \in k^{*} Q_{\geq 0}$. Esta relação é compatível com a concatenação e satisfaz a condição da cadeia descendente (Lema 2.11 [CS15]).

Com ajuda da relação $\preceq$, dado $n \geq 1$ definimos os subconjuntos de $k Q \otimes_{E} k \mathcal{A}_{n} \otimes_{E} k Q$

$$
\begin{aligned}
\mathcal{L}_{n}^{\prec}(\mu q) & :=\left\{\lambda a \otimes p \otimes c: a, c \in Q_{\geq 0}, p \in \mathcal{A}_{n}, \lambda a p c \preceq \mu q\right\}, \\
\mathcal{L}_{n}^{\prec}(\mu q) & :=\left\{\lambda a \otimes p \otimes c: a, c \in Q_{\geq 0}, p \in \mathcal{A}_{n}, \lambda a p c \prec \mu q\right\},
\end{aligned}
$$

e os subconjuntos de $A \otimes_{E} k \mathcal{A}_{n} \otimes_{E} A$

$$
\begin{aligned}
& \overline{\mathcal{L}}_{n}^{\prec}(\mu q):=\left\{\lambda \pi(b) \otimes p \otimes \pi\left(b^{\prime}\right): b, b^{\prime} \in \mathcal{B}, p \in \mathcal{A}_{n}, \lambda b p b^{\prime} \preceq \mu q\right\} \\
& \overline{\mathcal{L}}_{n}^{\prec}(\mu q):=\left\{\lambda \pi(b) \otimes p \otimes \pi\left(b^{\prime}\right): b, b^{\prime} \in \mathcal{B}, p \in \mathcal{A}_{n}, \lambda b p b^{\prime} \prec \mu q\right\}
\end{aligned}
$$

a fim de enunciar e mostrar alguns resultados que são base para a construção da resolução projetiva desejada.

Nota 8. Usando as definições acima é fácil ver que os homomorfismos $f_{n}$ e $S_{n}$ "preservam"combinações $\mathbb{Z}$-lineares de elementos dos subconjuntos definidos anteriormente. Mais formalmente,

$$
\begin{aligned}
f_{n+1}(x) & \in\left\langle\mathcal{L}_{n}^{\prec}(\mu q)\right\rangle_{\mathbb{Z}}, \quad \text { para todo } x \in \mathcal{L}_{n+1}^{\prec}(\mu q), e \\
S_{n}(x) & \in\left\langle\mathcal{L}_{n}^{\prec}(\mu q)\right\rangle_{\mathbb{Z}}, \quad \text { para todo } x \in \mathcal{L}_{n}^{\preceq}-1(\mu q) .
\end{aligned}
$$

Lema 15. Se $n \geq 1$ e $\mu q \in k^{*} Q_{\geq 0}$, então temos as seguintes inclusões

$$
\begin{gathered}
\pi_{n}\left(\mathcal{L}_{n}^{\prec}(\mu q)\right) \subseteq\left\langle\overline{\mathcal{L}}_{n}^{\prec}(\mu q)\right\rangle_{\mathbb{Z}} e \\
\pi_{n}\left(\mathcal{L}_{n}^{\prec}(\mu q)\right) \subseteq\left\langle\overline{\mathcal{L}}_{n}^{\prec}(\mu q)\right\rangle_{\mathbb{Z}}
\end{gathered}
$$

Lema 16. Dado $n \geq 1$ e $\mu q \in k^{*} Q_{\geq 0}$, temos as seguintes inclusões 
1. $\delta_{n}\left(\overline{\mathcal{L}}_{n}^{\preceq}(\mu q)\right) \subseteq\left\langle\overline{\mathcal{L}}_{n-1}^{\preceq}(\mu q)\right\rangle_{\mathbb{Z}}$,

2. $\delta_{n}\left(\overline{\mathcal{L}}_{n}^{\prec}(\mu q)\right) \subseteq\left\langle\overline{\mathcal{L}}_{n-1}^{\prec}(\mu q)\right\rangle_{\mathbb{Z}}$,

3. $s_{n}\left(\overline{\mathcal{L}}_{n-1}^{\preceq}(\mu q)\right) \subseteq\left\langle\overline{\mathcal{L}}_{n}^{\preceq}(\mu q)\right\rangle_{\mathbb{Z}}$,

4. $s_{n}\left(\overline{\mathcal{L}}_{n-1}^{\prec}(\mu q)\right) \subseteq\left\langle\overline{\mathcal{L}}_{n}^{\prec}(\mu q)\right\rangle_{\mathbb{Z}}$.

Demonstração. Vamos demonstrar somente a primeira afirmação. As outras afirmações têm uma demonstração similar. Seja $x=\lambda \pi(b) \otimes p \otimes \pi\left(b^{\prime}\right) \in \overline{\mathcal{L}}_{n}^{\preceq}(\mu q)$, então

$$
\delta_{n}(x)=\pi_{n-1}\left(f_{n}\left(i_{n}(x)\right)\right)=\pi_{n-1}\left(f_{n}\left(\lambda b \otimes p \otimes b^{\prime}\right)\right)
$$

Note que $\lambda b \otimes p \otimes b^{\prime} \in \mathcal{L}_{\bar{n}}^{\prec}(\mu q), \operatorname{logo} f_{n}\left(\lambda b \otimes p \otimes b^{\prime}\right) \in\left\langle\mathcal{L}_{n-1}^{\preceq}(\mu q)\right\rangle_{\mathbb{Z}}$ pela Nota(8). Pelo Lema(15) podemos concluir que $\delta_{n}(x) \in\left\langle\overline{\mathcal{L}}_{n-1}^{\prec}(\mu q)\right\rangle_{\mathbb{Z}}$.

Lema 17. Seja $n \geq 0$ e $\mu q \in k^{*} Q_{\geq 0}$. Se $x=\lambda a \otimes p \otimes c \in \mathcal{L}_{\bar{n}}^{\prec}(\mu q)$ é tal que $\pi_{n}^{\prime}(x)=0$, então $\pi_{n}(x) \in\left\langle\overline{\mathcal{L}}_{n}^{\prec}(\mu q)\right\rangle_{\mathbb{Z}}$.

Demonstração. Temos que $0=\pi_{n}^{\prime}(x)=\pi^{\prime}(a) \otimes p \otimes \pi^{\prime}(c)$, então $\pi^{\prime}(a)=0$ ou $\pi^{\prime}(c)=0$, isto é, $a \notin \mathcal{B}$ ou $c \notin \mathcal{B}$, o que implica $\beta(a) \prec a$ ou $\beta(c) \prec c$. Podemos supor sem perda de generalidade $\beta(a) \prec a$

Sejam $\beta(a)=\sum_{i} \lambda_{i} b_{i}$ e $\beta(c)=\sum_{j} \lambda_{j}^{\prime} b_{j}^{\prime}$. Como $\lambda_{i} b_{i} \prec a$ e $\lambda_{j} b_{j}^{\prime} \preceq c$ para quaisquer $i, j$, então $\lambda \lambda_{i} \lambda_{j}^{\prime} b_{i} p b_{j}^{\prime} \prec \lambda a p c \preceq \mu q$ para todo $i, j$ e portanto

$$
\sum_{i, j} \lambda \lambda_{i} \lambda_{j}^{\prime} \pi\left(b_{i}\right) \otimes p \otimes \pi\left(b_{j}^{\prime}\right) \in\left\langle\overline{\mathcal{L}}_{n}^{\prec}(\mu q)\right\rangle_{\mathbb{Z}}
$$

Computando $\pi_{n}(x)$ obtemos

$$
\pi_{n}(x)=\pi_{n}(\beta(x))=\pi_{n}\left(\sum_{i, j} \lambda \lambda_{i} \lambda_{j}^{\prime} b_{i} \otimes p \otimes b_{j}^{\prime}\right)=\sum_{i, j} \lambda \lambda_{i} \lambda_{j}^{\prime} \pi\left(b_{i}\right) \otimes p \otimes \pi\left(b_{j}^{\prime}\right)
$$

Corolário 7. Seja $n \geq 2, \mu q \in k^{*} Q_{\geq 0}$ e $x \in \overline{\mathcal{L}}_{n}^{\prec}(\mu q)$, então

1. $\delta_{n-1} \circ \delta_{n}(x) \in\left\langle\overline{\mathcal{L}}_{n-2}^{\prec}(\mu q)\right\rangle_{\mathbb{Z}}$,

2. $x-\delta_{n+1} \circ s_{n+1}(x)-s_{n} \circ \delta_{n} \in\left\langle\overline{\mathcal{L}}_{n}^{\prec}(\mu q)\right\rangle_{\mathbb{Z}}$.

Lema 18. Seja $n \in \mathbb{N}_{0}$, e seja $R=k$ ou $R=\mathbb{Z}$,

1. Se d : $A \otimes_{E} k \mathcal{A}_{n} \otimes_{E} A \rightarrow A \otimes_{E} k \mathcal{A}_{n-1} \otimes_{E} A$ é um homomorfismo de A-bimódulos tal que $\left(d-\delta_{n}\right)(1 \otimes p \otimes 1) \in\left\langle\overline{\mathcal{L}}_{n-1}^{\prec}(p)\right\rangle_{R}$ para todo $p \in \mathcal{A}_{n}$, então dado $x \in\left\langle\overline{\mathcal{L}}_{n}^{\prec}(\mu q)\right\rangle_{R},\left(d-\delta_{n}\right)(x) \in$ $\left\langle\overline{\mathcal{L}}_{n-1}^{\prec}(\mu q)\right\rangle_{R}$ para todo $\mu q \in k^{*} Q_{\geq 0}$.

2. Se $\rho: A \otimes_{E} k \mathcal{A}_{n-1} \otimes_{E} A \rightarrow A \otimes_{E} k \mathcal{A}_{n} \otimes_{E} A$ é um homomorfismo de $A-E$-bimódulos tal que $\left(\rho-s_{n}\right)(1 \otimes p \otimes \pi(b)) \in\left\langle\overline{\mathcal{L}}_{n}^{\prec}(p b)\right\rangle_{R}$ para todo $p \in \mathcal{A}_{n}$ e $b \in \mathcal{B}$, então para todo $x \in\left\langle\overline{\mathcal{L}}_{n}^{\prec}(\mu q)\right\rangle_{R}$, $\left(\rho-s_{n}\right)(x) \in\left\langle\overline{\mathcal{L}}_{n}^{\prec}(\mu q)\right\rangle_{R}$ para todo $\mu q \in k^{*} Q_{\geq 0}$. 
Demonstração. Seja $\mu q \in k^{*} Q_{\geq 0}$ e $x \in\left\langle\overline{\mathcal{L}}_{n}^{\prec}(\mu q)\right\rangle_{R}$. Queremos mostrar que $\left(d-\delta_{n}\right)(x) \in\left\langle\overline{\mathcal{L}}_{n-1}^{\prec}(\mu q)\right\rangle_{R}$. É suficiente mostrar a afirmação para $x=\lambda \pi(b) \otimes p \otimes \pi\left(b^{\prime}\right) \in \overline{\mathcal{L}}_{n}^{\prec}(\mu q)$.

Pela hipótese, $\left(d-\delta_{n}\right)(1 \otimes p \otimes 1) \in\left\langle\overline{\mathcal{L}}_{n-1}^{\prec}(p)\right\rangle_{R}$, logo $\left(d-\delta_{n}\right)(x)=\lambda \pi(b)\left(d-\delta_{n}\right)(1 \otimes p \otimes 1) \pi\left(b^{\prime}\right)$ pertence a $\left\langle\overline{\mathcal{L}}_{n-1}^{\prec}\left(\lambda b p b^{\prime}\right)\right\rangle_{R}$ como consequência do Lema(15). Como $\left\langle\overline{\mathcal{L}}_{n-1}^{\prec}\left(\lambda b p b^{\prime}\right)\right\rangle_{R} \subseteq\left\langle\overline{\mathcal{L}}_{n-1}^{\prec}(\mu q)\right\rangle_{R}$, então $\left(d-\delta_{n}\right)(x) \in\left\langle\overline{\mathcal{L}}_{n-1}^{\prec}(\mu q)\right\rangle_{R}$.

Proposição 17. Seja $n \in \mathbb{N}_{0}$. Suponha que para todo $i \in\{1, \ldots, n\}$ existem homomorfismos de $A$-bimódulos $d_{i}: A \otimes_{E} k \mathcal{A}_{i} \otimes_{E} A \rightarrow A \otimes_{E} k \mathcal{A}_{i-1} \otimes_{E} A$, e homomorfismos de $A-E$-bimódulos $\rho_{i}: A \otimes_{E} k \mathcal{A}_{i-1} \otimes_{E} A \rightarrow A \otimes_{E} k \mathcal{A}_{i} \otimes_{E} A$. Denote $d_{0}=\mu$ e defina $\rho_{0}: A \rightarrow A \otimes_{E} A$ como $\rho(a)=a \otimes 1$. Se

(i) $d_{i-1} \circ d_{i}=0$ para todo $i \in\{1, \ldots, n\}$,

(ii) $\left(d_{i}-\delta_{i}\right)(1 \otimes q \otimes 1) \in\left\langle\overline{\mathcal{L}}_{i-1}^{\prec}(q)\right\rangle_{R}$ para todo $i \in\{1, \ldots, n\}$ e todo $q \in \mathcal{A}_{i}$,

(iii) para todo $i \in\{0, \ldots, n-1\}$ e todo $x \in \otimes_{E} k \mathcal{A}_{i} \otimes_{E} A, x=d_{i+1} \circ \rho_{i+1}(x)+\rho_{i} \circ d_{i}(x)$,

(iv) $\left(\rho_{i}-s_{i}\right)(1 \otimes q \otimes \pi(b)) \in\left\langle\overline{\mathcal{L}}_{i}^{\prec}(q b)\right\rangle_{R}$ para todo $i \in\{1, \ldots, n\}$ para todo $q \in \mathcal{A}_{i}$ e todo $b \in \mathcal{B}$, então

1. Existe um homomorfismo de A-bimódulos $d_{n+1}: A \otimes_{E} k \mathcal{A}_{n+1} \otimes_{E} A \rightarrow A \otimes_{E} k \mathcal{A}_{n} \otimes_{E} A$ tal que

(a) $d_{n} \circ d_{n+1}=0$

(b) $\left(d_{n+1}-\delta_{n+1}\right)(1 \otimes q \otimes 1) \in\left\langle\overline{\mathcal{L}}_{n}^{\prec}(q)\right\rangle_{R}$.

2. Existe um homomorfismo $\rho_{n+1}: A \otimes_{E} k \mathcal{A}_{n} \otimes_{E} A \rightarrow A \otimes_{E} k \mathcal{A}_{n+1} \otimes_{E} A$ de $A-E$-bimódulos tal que

(c) para todo $x \in A \otimes_{E} k \mathcal{A}_{n} \otimes_{E} A, x=d_{n+1} \circ \rho_{n+1}(x)+\rho_{n} \circ d_{n}(x) e$

(d) para todo $q \in \mathcal{A}_{n}$ e todo $b \in \mathcal{B},\left(\rho_{n+1}-s_{n+1}\right)(1 \otimes q \otimes \pi(b)) \in\left\langle\mathcal{L}_{n+1}^{\prec}(q b)\right\rangle_{R}$.

Demonstração. $\quad(1)$ Seja $q \in \mathcal{A}_{n+1}$. Note que $\delta_{n+1}(1 \otimes q \otimes 1) \in\left\langle\overline{\mathcal{L}}_{n}^{\prec}(q)\right\rangle_{\mathbb{Z}}$ pois $1 \otimes q \otimes 1 \in \overline{\mathcal{L}}_{n+1}^{\prec}(q)$.

Queremos mostrar que $d_{n}\left(\delta_{n+1}(1 \otimes q \otimes 1)\right) \in\left\langle\overline{\mathcal{L}}_{n-1}^{\prec}(q)\right\rangle_{R}$. Como

$$
d_{n}\left(\delta_{n+1}(1 \otimes q \otimes 1)\right)=\delta_{n} \circ \delta_{n+1}(1 \otimes q \otimes 1)+\left(d_{n}-\delta_{n}\right)\left(\delta_{n+1}(1 \otimes q \otimes 1)\right)
$$

é suficiente mostrar que $\left(d_{n}-\delta_{n}\right)\left(\delta_{n+1}(1 \otimes q \otimes 1)\right) \in\left\langle\overline{\mathcal{L}}_{n-1}^{\prec}(q)\right\rangle_{R}$ e $\delta_{n} \circ \delta_{n+1}(1 \otimes q \otimes 1) \in$ $\left\langle\overline{\mathcal{L}}_{n-1}^{\prec}(q)\right\rangle_{R}$. Isto é consequência do Lema(18), o Corolário(7) e o fato de $1 \otimes q \otimes 1$ pertencer a $\overline{\mathcal{L}}_{n+1}^{\preceq}(q)$.

Definamos $\tilde{\mathrm{d}}_{n+1}: A \times k \mathcal{A}_{n+1} \times A \rightarrow A \otimes_{E} k \mathcal{A}_{n} \otimes_{E} A$ por

$$
\tilde{\mathrm{d}}_{n+1}(a, q, c)=a \delta_{n+1}(1 \otimes q \otimes 1) c-a \rho_{n}\left(d_{n}\left(\delta_{n+1}(1 \otimes q \otimes 1)\right)\right) c,
$$

para todo $a, c \in A$ e $q \in \mathcal{A}_{n+1}$. A aplicação $\tilde{\mathrm{d}}_{n+1}$ é $E$-multilinear e balanceada, então induz uma única aplicação

$$
d_{n+1}: A \otimes_{E} k \mathcal{A}_{n+1} \otimes_{E} A \rightarrow A \otimes_{E} k \mathcal{A}_{n} \otimes_{E} A,
$$

a qual é um homomorfismo de $A$-bimódulos. 
Escrevendo $\rho_{n}=s_{n}+\left(\rho_{n}-s_{n}\right)$ e fazendo $x=d_{n}\left(\delta_{n+1}(1 \otimes q \otimes 1)\right)$ temos

$$
\left(d_{n+1}-\delta_{n+1}\right)(1 \otimes q \otimes 1)=-\rho_{n} \circ d_{n} \circ \delta_{n+1}(1 \otimes q \otimes 1)=-s_{n}(x)-\left(\rho_{n}-s_{n}\right)(x) .
$$

Já mostramos que $d_{n}\left(\delta_{n+1}(1 \otimes q \otimes 1)\right) \in\left\langle\overline{\mathcal{L}}_{n-1}^{\prec}(q)\right\rangle_{R}$, logo $s_{n}(x) \in\left\langle\overline{\mathcal{L}}_{n}^{\prec}(q)\right\rangle_{R}$ pelo Lema(16) e usando a hipótese $(i v)$ e o Lema(18) obtemos que $\left(\rho_{n}-s_{n}\right)(x) \in\left\langle\overline{\mathcal{L}}_{n}^{\prec}(q)\right\rangle_{R}$. Portanto $\left(d_{n+1}-\delta_{n+1}\right)(1 \otimes q \otimes 1) \in\left\langle\overline{\mathcal{L}}_{n}^{\prec}(q)\right\rangle_{R}$.

Como $x=d_{n}\left(\delta_{n+1}(1 \otimes q \otimes 1)\right) \in A \otimes_{E} k \mathcal{A}_{n-1} \otimes_{E} A$, por $(i i i)$ com $i=n-1, x=d_{n} \circ \rho_{n}(x)+$ $\rho_{n-1} \circ d_{n-1}(x)$. Logo

$$
d_{n} \circ \delta_{n+1}(1 \otimes q \otimes 1)=d_{n} \circ \rho_{n} \circ d_{n} \circ \delta_{n+1}(1 \otimes q \otimes 1),
$$

e temos provado que $d_{n} \circ d_{n+1}=0$.

(1) Seja $q \in \mathcal{A}_{n+1}$ e $b \in \mathcal{B}$. Temos que

$$
\begin{aligned}
1 \otimes q \otimes \pi(b)-\rho_{n} \circ d_{n}(1 \otimes q \otimes \pi(b))= & 1 \otimes q \otimes \pi(b)-\rho_{n} \delta_{n}(1 \otimes q \otimes \pi(b)) \\
& -\rho_{n} \circ\left(d_{n}-\delta_{n}\right)(1 \otimes q \otimes \pi(b))
\end{aligned}
$$

Note que $1 \otimes q \otimes \pi(b) \in \overline{\mathcal{L}}_{n}^{\prec}(q b), \operatorname{logo}\left(d_{n}-\delta_{n}\right)(x) \in\left\langle\overline{\mathcal{L}}_{n-1}^{\prec}(q b)\right\rangle_{R}$ pelo Lema(18). Expressando $\rho_{n}$ da forma $s_{n}+\left(\rho_{n}-s_{n}\right)$ e usando os $\operatorname{Lemas}(16,18)$ podemos concluir que $\rho_{n} \circ\left(d_{n}-\delta_{n}\right)(1 \otimes$ $q \otimes \pi(b)) \in\left\langle\overline{\mathcal{L}}_{n}^{\prec}(q b)\right\rangle_{R}$. Neste caso escrevemos

$$
\left(i d-\rho_{n} \circ \delta_{n}+\rho_{n} \circ\left(d_{n}-\delta_{n}\right)\right)(1 \otimes q \otimes \pi(b)) \equiv i d-\rho_{n} \circ \delta_{n}(1 \otimes q \otimes \pi(b)) \bmod \left\langle\overline{\mathcal{L}}_{n}^{\prec}(q b)\right\rangle_{R} .
$$

Também temos as seguintes congruências módulo $\left\langle\overline{\mathcal{L}}_{n}^{\prec}(q b)\right\rangle_{r}$

$$
\begin{aligned}
\left(i d-\rho_{n} \circ \delta_{n}\right)(1 \otimes q \otimes \pi(b)) & \equiv\left(i d-s_{n} \circ \delta_{n}\right)(1 \otimes q \otimes \pi(b)) \bmod \left\langle\overline{\mathcal{L}}_{n}^{\prec}(q b)\right\rangle_{R} \\
& \equiv \delta_{n+1} \circ s_{n+1}(1 \otimes q \otimes \pi(b)) \bmod \left\langle\overline{\mathcal{L}}_{n}^{\prec}(q b)\right\rangle_{R} \\
& \equiv d_{n+1} \circ s_{n+1}(1 \otimes q \otimes \pi(b)) \bmod \left\langle\overline{\mathcal{L}}_{n}^{\prec}(q b)\right\rangle_{R} .
\end{aligned}
$$

Pelo anterior, existe $\xi \in\left\langle\overline{\mathcal{L}}_{n}^{\prec}(q b)\right\rangle_{R}$ tal que

$$
\left(i d-\rho_{n} \circ d_{n}\right)(1 \otimes q \otimes \pi(b))=d_{n+1} \circ s_{n+1}(1 \otimes q \otimes \pi(b))+\xi .
$$

Vamos ver que $\xi \in \operatorname{ker}\left(d_{n}\right)$. Para isto, basta mostrar que $d_{n}\left(\left(i d-\rho_{n} \circ d_{n}\right)(1 \otimes q \otimes \pi(b))\right)=0$ e $d_{n}\left(d_{n+1} \circ s_{n+1}(1 \otimes q \otimes \pi(b))\right)=0$. Se denotamos $1 \otimes q \otimes \pi(b)$ por $x$ então, usando $(i i i)$ e $(i)$ obtemos

$$
\begin{aligned}
d_{n}\left(x-\rho_{n} \circ d_{n}(x)\right) & =d_{n}(x)-\left(d_{n} \rho_{n}\right)\left(d_{n}(x)\right) \\
& =d_{n}(x)-\left(d_{n}(x)-\rho_{n-1} d_{n-1}\left(d_{n}(x)\right)\right) \\
& =0
\end{aligned}
$$

Por outro lado $d_{n}\left(d_{n+1} \circ s_{n+1}(1 \otimes q \otimes \pi(b))\right)=0$ pois estamos supondo $d_{n} \circ d_{n=1}=0$.

Como $\preceq$ satisfaz a condição da cadeia descendente podemos usar indução sobre $\left(k^{*} Q_{\geq 0}, \preceq\right)$. Se não existe $\lambda p \in k^{*} Q_{\geq 0}$ tal que $\lambda p \prec q b$, então $\xi=0$ e definimos $\rho_{n+1}(1 \otimes q \otimes \pi(b)):=$ $s_{n+1}(1 \otimes q \otimes \pi(b))$. Agora, suponha que $\rho_{n+1}(\xi)$ está definido. A igualdade $d_{n}(\xi)=0$ implica $\xi=d_{n+1} \circ \rho_{n+1}(\xi)$

$$
\left(i d-\rho_{n} \circ d_{n}\right)(1 \otimes q \otimes \pi(b))=d_{n+1}\left(s_{n+1}(1 \otimes q \otimes \pi(b))+\rho_{n+1}(\xi)\right) .
$$


Definimos $\rho_{n+1}(1 \otimes q \otimes \pi(b)):=s_{n+1}(1 \otimes q \otimes \pi(b))+\rho_{n+1}(\xi)$.

Teorema 13. Sejam $d_{0}:=\delta_{0}$ e $d_{1}:=\delta_{1}$. Dado $N \in \mathbb{N}_{0}$ e homomorfismos de A-bimódulos $d_{i}$ : $A \otimes_{E} k \mathcal{A}_{i} \otimes_{E} A \rightarrow A \otimes_{E} k \mathcal{A}_{i-1} \otimes_{E} A$ para $2 \leq i \leq N$. Se

(1) $d_{i-1} \circ d_{i}=0$ para todo $i$, com $2 \leq i \leq N$,

(2) $\left(d_{i}-\delta_{i}\right)(1 \otimes q \otimes 1) \in\left\langle\overline{\mathcal{L}}_{i-1}^{\prec}(q)\right\rangle_{k}$ para todo $2 \leq i \leq N$ e todo $q \in \mathcal{A}_{i}$,

então o complexo

$$
A \otimes_{E} k \mathcal{A}_{N} \otimes_{E} A \stackrel{d_{N}}{\longrightarrow} \cdots \stackrel{d_{2}}{\longrightarrow} A \otimes_{E} k \mathcal{A}_{1} \otimes_{E} A \stackrel{d_{1}}{\longrightarrow} A \otimes_{E} A \stackrel{d_{0}}{\longrightarrow} A \rightarrow 0
$$

é exato.

Demonstração. Vamos mostrar que existe uma aplicação de $A-E$-bimódulos $\rho_{1}: A \otimes_{E} k \mathcal{A}_{0} \otimes_{E} A \rightarrow$ $A \otimes_{E} k \mathcal{A}_{1} \otimes_{E} A$ que satisfaz $d_{1} \circ \rho_{1}+\rho_{0} \circ d_{0}=i d$, com $d_{0}=\mu$ e $\rho_{0}(a)=s_{0}(a)=a \otimes 1$ para todo $a \in A$.

Dado $b=b_{k} \cdots b_{1} \in \mathcal{B}$, com $b_{i} \in Q_{1}$ para todo $1 \leq i \leq k$, temos que

$$
s_{1}(1 \otimes \pi(b))=-\sum_{i} \pi\left(b_{k} \cdots b_{k-i+1}\right) \otimes b_{k-i} \otimes \pi\left(b_{k-i-1} \cdots b_{1}\right)
$$

Por um lado $1 \otimes \pi(b)-\pi(b) \otimes 1=1 \otimes \pi(b)-s_{0}\left(d_{0}(1 \otimes \pi(b))\right)$, e por outro lado temos que $1 \otimes \pi(b)-\pi(b) \otimes 1=\delta_{1}\left(s_{1}(1 \otimes \pi(b))\right), \operatorname{logo} 1 \otimes \pi(b)-s_{0}(1 \otimes \pi(b))=\delta_{1}\left(s_{1}(1 \otimes \pi(b))\right)$. Pela hipótese, $\left(d_{1}-\delta_{1}\right)(1 \otimes \pi(b)) \in\left\langle\overline{\mathcal{L}}_{0}^{\prec}(b)\right\rangle_{k}$, portanto existe $\xi \in\left\langle\overline{\mathcal{L}}_{0}^{\prec}(q)\right\rangle_{k}$ tal que

$$
1 \otimes \pi(b)-s_{0}\left(d_{0}(1 \otimes \pi(b))\right)=d_{1}\left(s_{1}(1 \otimes \pi(b))\right)+\xi .
$$

Logo $d_{0}(\xi)=0$. Suponha que não existe $\lambda p \in k^{*} Q_{\geq 0}$ tal que $\lambda p \prec b$. Neste caso, definimos $\rho_{1}(1 \otimes \pi(b))=s_{1}(1 \otimes \pi(b))$. Agora, suponha que $\rho_{0}(\xi)$ está definido para todo $\xi$ tal que $d_{0}(\xi)=0$. Como $\xi=d_{1}\left(\rho_{1}(\xi)\right)$, podemos definir $\rho_{1}(1 \otimes \pi(b)):=s_{1}(1 \otimes \pi(b))+\rho_{1}(\xi)$.

Por indução e a Proposição 17. obtemos uma retração de homotopia do complexo (3.3) provando que este é exato.

Teorema 14. Existem homomorfismos de A-bimódulos $d_{i}: A \otimes_{E} k \mathcal{A}_{i} \otimes_{E} A \rightarrow A \otimes_{E} k \mathcal{A}_{i-1} \otimes_{E} A$ para $i \geq 1$ e $d_{0}: A \otimes_{E} A \rightarrow A$ tal que

(1) $d_{i-1} \circ d_{i}=0$ para todo $i \geq 1$,

(2) $\left(d_{i}-\delta_{i}\right)(1 \otimes q \otimes 1) \in\left\langle\overline{\mathcal{L}}_{i-1}^{\prec}(q)\right\rangle_{\mathbb{Z}}$ para todo $i \geq 0$ e todo $q \in \mathcal{A}_{i}$,

Demonstração. Mostramos anteriormente que $1 \otimes \pi(b)=\left(s_{0} \circ d_{0}+\delta_{1} \circ s_{1}\right)(1 \otimes \pi(b))$, logo $s_{0} \circ d_{0}+$ $\delta_{1} \circ s_{1}=i d_{A \otimes_{E} A}$. O resultado segue da Proposição 17 fixando $d_{1}=\delta_{1}$.

Observe que conseguimos uma resolução projetiva de $A$ como $A$-bimódulo onde os bimódulos projetivos tem uma correspondência bijetiva com os bimódulos projetivos aparecendo na resolução minimal de $A_{S}$. Isto implica o seguinte resultado.

Corolário 8. Se $A_{S}$ tem dimensão global finita então A tem dimensão global finita.

Demonstração. $A_{S}$ tem dimensão global $n$ se e somente se $\mathcal{A}_{n+1}=0$ e nesse caso a resolução de $A$ que descrevemos é finita e portanto $A$ tem dimensão global finita. 
Essa observação foi usada por Green e posteriormente por Green e Sybille para descrever uma variedade algébrica das álgebras sobre um carcás fixo que tem a mesma álgebra monomial associada. Este é um assunto que pretendemos investigar no nosso trabalho a seguir.

\subsection{Primeiros homomorfismos da resolução}

Seja $A=k Q / I$ e $\mathcal{R}$ um sistema de redução satisfazendo a condição do Diamante para $I$. Usando a definição de $\delta_{0}: A \otimes_{E} A \rightarrow A$ e $\delta_{1}: A \otimes_{E} k \mathcal{A}_{1} \otimes_{E} A \rightarrow A \otimes_{E} A$, temos que para todo $a, c \in k Q$ e $\alpha \in Q_{1}$,

$$
\delta_{0}(\pi(a) \otimes \pi(c))=\pi(a c)
$$

$\mathrm{e}$

$$
\delta_{1}(\pi(a) \otimes \alpha \otimes \pi(c))=\pi(a \alpha) \otimes \pi(c)-\pi(a) \otimes \pi(\alpha c) .
$$

Seja $\phi_{1}: k Q \rightarrow A \otimes_{E} k \mathcal{A}_{1} \otimes_{E} A$ a única aplicação $k$-linear tal que

$$
\phi_{1}(c)=\sum_{n}^{i=1} \pi\left(c_{n} \cdots c_{i+1}\right) \otimes c_{i} \otimes \pi\left(c_{i-1} \cdots c_{1}\right)
$$

para $c \in Q_{\geq 0}, c=c_{n} \cdots c_{1}$, com $c_{i} \in Q_{1}$ para todo $i, 1 \leq i \leq n$.

Dada uma redução básica $r=r_{a, s, c}$, definimos $\phi_{2}(r,-): k Q \rightarrow A \otimes_{E} k \mathcal{A}_{2} \otimes_{E} A$ como a única aplicação $k$-linear tal que, dado $p \in Q \geq 0$,

$$
\phi_{2}(r, p)= \begin{cases}\pi(a) \otimes s \otimes \pi(c) & \text { se } p=a s c \\ 0 & \text { caso contrario. }\end{cases}
$$

Com o anterior, podemos definir de maneira recursiva $\phi_{2}(r,-)$ para qualquer redução $r$. Seja $r=$ $\left(r_{n}, \ldots, r_{1}\right)$ uma redução com $r_{i}$ uma redução básica para $1 \leq i \leq n$ e denotemos $r^{\prime}=\left(r_{n}, \ldots, r_{2}\right)$. Definimos $\phi_{2}(r,-): k Q \rightarrow A \otimes_{E} k \mathcal{A}_{2} \otimes_{E} A$ como a única aplicação $k$-linear tal que, dado $p \in Q_{\geq 0}$,

$$
\phi_{2}(r, p)=\phi_{2}\left(r_{1}, p\right)+\phi_{2}\left(r^{\prime}, r_{1}(p)\right) .
$$

Definimos um homomorfismo de $A$-bimódulos $d_{2}: A \otimes_{E} k \mathcal{A}_{2} \otimes_{E} A \rightarrow A \otimes_{E} k \mathcal{A}_{1} \otimes_{E} A$ por

$$
d_{2}(1 \otimes s \otimes 1)=\phi_{1}(s)-\phi_{1}(\beta(s)),
$$

para todo $s \in \mathcal{A}_{2}$.

Lema 19. Seja $p \in Q_{\geq 0}$ e $x \in k Q$ tal que $x \prec p$. Para toda redução $r, \phi_{2}(r, x) \in\left\langle\overline{\mathcal{L}}_{2}^{\prec}(p)\right\rangle_{\mathbb{Z}}$.

Lema 20. Para todo $x \in A \otimes_{E} k \mathcal{A}_{2} \otimes_{E} A$, x pertence a $k e r\left(\delta_{1} \circ d_{2}\right)$.

Demonstração. Seja $x \in A \otimes_{E} k \mathcal{A}_{2} \otimes_{E} A$. Como $\delta_{1}$ e $d_{2}$ são homomorfismos de bimódulos, podemos assumir $x$ da forma $1 \otimes s \otimes 1$, com $s \in \mathcal{A}_{2}$, logo

$$
\delta_{1}\left(d_{2}(1 \otimes s \otimes 1)\right)=\delta_{1}\left(\phi_{1}(s)-\phi_{1}(\beta(s))\right)=\pi(s) \otimes 1-1 \otimes \pi(s)-\pi(\beta(s)) \otimes 1+1 \otimes \pi(\beta(s))=0 .
$$

Usando a definição de $\phi_{1}$ e a $k$-linearidade de $\phi_{1}$ e $\pi$ temos o seguinte resultado.

Lema 21. Dados $a, c \in Q_{\geq 0}$ e $p=\sum_{i=1}^{n} \in k Q$, com $p_{i} \in Q_{\geq 0}$ para $1 \leq i \leq n$, temos que

$$
\phi_{1}(a p c)=\phi_{1}(a) \pi(p c)+\pi(a) \phi_{1}(p) \pi(c)+\pi(a p) \phi_{1}(c) .
$$


Lema 22. Dado $p \in Q_{\geq 0}$ e uma redução $r=\left(r_{n}, \ldots, r_{1}\right)$, com $r_{i}$ uma redução básica para $1 \leq i \leq n$, temos que

$$
d_{2}\left(\phi_{2}(r(p))\right)=\phi_{1}(p)-\phi_{1}(r(p)) .
$$

Demonstração. Vamos fazer a demonstração por indução sobre $n$.

Seja $r=r_{1}=r_{a_{1}, s_{1}, c_{1}}$ uma redução básica. Se $p \neq a_{1} s_{1} c_{1}$ então $r_{1}(p)=p$ e $\phi_{2}(r, p)=0$, logo a equação (3.4) é satisfeita. Se $p=a_{1} s_{1} c_{1}$ então $\phi_{2}\left(r_{1}, p\right)=\pi\left(a_{1}\right) \otimes s_{1} \otimes \pi\left(c_{1}\right)$ e $r_{1}(p)=a_{1} \beta\left(s_{1}\right) c_{1}$. Logo,

$$
\begin{aligned}
d_{2}\left(\phi_{2}\left(r_{1}, p\right)\right)+\phi_{1}\left(r_{1}(p)\right) & =d_{2}\left(\pi\left(a_{1}\right) \otimes s_{1} \otimes \pi\left(c_{1}\right)\right)+\phi_{1}\left(a_{1} \beta\left(s_{1}\right) c_{1}\right) \\
& =\pi\left(a_{1}\right) \phi_{1}\left(s_{1}\right) \pi\left(c_{1}\right)-\pi\left(a_{1}\right) \phi_{1}\left(\beta\left(s_{1}\right)\right) \pi\left(c_{1}\right)+\phi_{1}\left(a_{1} \beta\left(s_{1}\right) c_{1}\right)
\end{aligned}
$$

Pelo Lema 21.

$$
\phi_{1}\left(a_{1} \beta\left(s_{1}\right) c_{1}\right)=\phi_{1}\left(a_{1}\right) \pi\left(\beta\left(s_{1}\right) c_{1}\right)+\pi\left(a_{1}\right) \phi_{1}\left(\beta\left(s_{1}\right)\right) \pi\left(c_{1}\right)+\pi\left(a_{1} \beta\left(s_{1}\right)\right) \phi_{1}\left(c_{1}\right) .
$$

Então por (3.5) e (3.6) temos que

$$
\begin{aligned}
d_{2}\left(\phi_{2}\left(r_{1}, p\right)\right)+\phi_{1}\left(r_{1}(p)\right) & =\pi\left(a_{1}\right) \phi_{1}\left(s_{1}\right) \pi\left(c_{1}\right)+\phi_{1}\left(a_{1}\right) \pi\left(\beta\left(s_{1}\right) c_{1}\right)+\pi\left(a_{1} \beta\left(s_{1}\right)\right) \phi_{1}\left(c_{1}\right) \\
& =\pi\left(a_{1}\right) \phi_{1}\left(s_{1}\right) \pi\left(c_{1}\right)+\phi_{1}\left(a_{1}\right) \pi\left(s_{1} c_{1}\right)+\pi\left(a_{1} s_{1}\right) \phi_{1}\left(c_{1}\right) .
\end{aligned}
$$

Esta última expressão é igual a $\phi_{1}\left(a_{1} s_{1} c_{1}\right)=\phi_{1}(p)$ pelo Lema 21. Portanto

$$
d_{2}\left(\phi_{2}\left(r_{1}, p\right)\right)+\phi_{1}\left(r_{1}(p)\right)=\phi_{1}(p) .
$$

Agora vamos supor que o resultado vale para $n-1$. Vamos notar $r^{\prime}=\left(r_{n}, \ldots, r_{2}\right)$. Sabemos pela definição de $\phi_{2}$ que $d_{2}\left(\phi_{2}(r, p)\right)=d_{2}\left(\phi_{2}\left(r_{1}, p\right)\right)+d_{2}\left(\phi_{2}\left(r^{\prime}, r_{1}(p)\right)\right)$, logo

$$
\begin{aligned}
d_{2}\left(\phi_{2}(r, p)\right)+\phi_{1}(r(p)) & =d_{2}\left(\phi_{2}\left(r_{1}, p\right)\right)+d_{2}\left(\phi_{2}\left(r^{\prime}, r_{1}(p)\right)\right)+\phi_{1}\left(r^{\prime}\left(r_{1}(p)\right)\right) \\
& =d_{2}\left(\phi_{2}\left(r_{1}, p\right)\right)+\phi_{1}\left(r_{1}(p)\right) \\
& =\phi_{1}(p) .
\end{aligned}
$$

Seja $p \in \mathcal{A}_{3}$. Podemos expressar $p$ como uma ambiguidade a esquerda e a direita por $p=$ $u_{0} u_{1} u_{2}=v_{2} v_{1} v_{0}$, onde $u_{0} u_{1}$ e $v_{1} v_{0}$ pertencem a $\mathcal{A}_{2}$ e são as únicas 2-ambiguidades que dividem $p$. Seja $r=r_{a, s, c}$ uma redução básica tal que $r(p) \neq p$, logo $s=u_{0} u_{1}$ ou $s=v_{1} v_{0}$. Dada uma redução $r=\left(r_{n}, \ldots, r_{1}\right)$, dizemos que $r$ começa à esquerda de $p$ se $r_{1}=r_{a, s, c}, s=u_{0} u_{1}$ e $a s c=p$,e dizemos que $r$ começa à direita de $p$ se $r_{1}=r_{a, s, c}, s=v_{1} v_{0}$ e $a s c=p$.

Considere a aplicação $\tilde{\mathrm{d}}_{3}: A \otimes_{E} k \mathcal{A}_{3} \otimes_{E} A \rightarrow A \otimes_{E} k \mathcal{A}_{2} \otimes_{E} A$ definida por

$$
\tilde{\mathrm{d}}_{3}(x, p, y)=x \phi_{2}\left(t^{p}, p\right) y-x \phi_{2}\left(r^{p}, p\right) y
$$

para todo $x, y \in A$. Usando a definição de $\phi_{2}$ podemos concluir que

$$
\tilde{\mathrm{d}}_{3}(x e, p, y)=\tilde{\mathrm{d}}_{3}(x, p e, y)=\tilde{\mathrm{d}}_{3}(x, p, e y),
$$

para todo $e \in E$. Logo, $\tilde{\mathrm{d}}_{3}$ é multilinear e induz uma aplicação $d_{3}: A \otimes_{E} k \mathcal{A}_{3} \otimes_{E} A \rightarrow A \otimes_{E} k \mathcal{A}_{2} \otimes_{E} A$. Esta aplicação é um morfismo de $A$-bimódulos dado por $d_{3}(1 \otimes p \otimes 1)=\phi_{2}\left(t^{p}, p\right)-\phi_{2}\left(r^{p}, p\right)$ para todo $p \in \mathcal{A}_{3}$.

Proposição 18. Sejam $\left\{r^{p}\right\}_{p \in \mathcal{A}_{3}}$ e $\left\{t^{p}\right\}_{p \in \mathcal{A}_{3}}$ dois conjuntos de reduções tais que $r^{p}(p)$ e $t^{p}(p)$

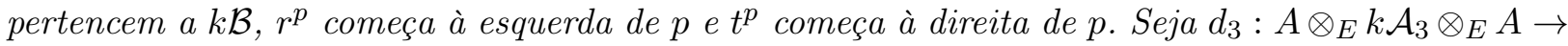


$A \otimes_{E} k \mathcal{A}_{2} \otimes_{E} A$ a aplicação de $A$-bimódulos definida como acima. A sequência

$$
\begin{aligned}
& A \otimes_{E} k \mathcal{A}_{3} \otimes_{E} A \stackrel{d_{3}}{\longrightarrow} A \otimes_{E} k \mathcal{A}_{2} \otimes_{E} A \stackrel{d_{2}}{\longrightarrow} A \otimes_{E} k \mathcal{A}_{1} \otimes_{E} A \stackrel{\delta_{1}}{\longrightarrow} A \otimes_{E} A \stackrel{\delta_{0}}{\longrightarrow} A \rightarrow 0, \\
& \text { é exata. }
\end{aligned}
$$

Demonstração. Vamos mostrar primeiro que a sequência dada em (3.7) é um complexo.

$$
\delta_{0}\left(\delta_{1}(\pi(a) \otimes \alpha \otimes \pi(c))\right)=\delta_{0}(\pi(a \alpha) \otimes \pi(c)-\pi(a) \otimes \pi(\alpha c))=\pi(a \alpha c)-\pi(a \alpha c)=0 .
$$

E já mostramos no Lema 20. que $\delta_{1} \circ d_{2}=0$.

Seja $p \in \mathcal{A}_{3}$. Usando o Lema 22. temos que

$$
\begin{aligned}
d_{2}\left(d_{3}(1 \otimes p \otimes 1)\right) & =d_{2}\left(\phi_{2}\left(t^{p}, p\right)-\phi_{2}\left(r^{p}, p\right)\right) \\
& =\phi_{1}(p)-\phi_{1}\left(t^{p}(p)\right)-\phi_{1}(p)+\phi_{1}\left(r^{p}(p)\right) \\
& =\phi_{1}\left(r^{p}(p)\right)-\phi_{1}\left(t^{p}(p)\right) .
\end{aligned}
$$

$\operatorname{Mas} \phi_{1}\left(r^{p}(p)\right)-\phi_{1}\left(t^{p}(p)\right)=\phi_{1}(\beta(p))-\phi_{1}(\beta(p)), \log 0 d_{2} \circ d_{3}=0$.

Pelo Teorema 13. basta mostrar que $d_{2}(1 \otimes s \otimes 1)-\delta_{2}(1 \otimes s \otimes 1) \in\left\langle\overline{\mathcal{L}}_{1}^{\prec}(s)\right\rangle_{k}$ para todo $s \in \mathcal{A}_{2}$ e $\left(d_{3}-\delta_{3}\right)(1 \otimes p \otimes 1) \in\left\langle\overline{\mathcal{L}}_{2}^{\prec}(p)\right\rangle_{k}$ para todo $p \in \mathcal{A}_{3}$ para mostrar a exatidão do complexo (3.7).

$\delta_{2}(1 \otimes s \otimes 1)=\phi_{1}(\beta(s))$, e como $\beta(s) \prec s$ então $\phi_{1}(\beta(s)) \in\left\langle\overline{\mathcal{L}}_{1}^{\prec}(s)\right\rangle_{k}$. Portanto

$$
d_{2}(1 \otimes s \otimes 1)-\delta_{2}(1 \otimes s \otimes 1)=-\phi_{1}(\beta(s)) \in\left\langle\overline{\mathcal{L}}_{1}^{\prec}(s)\right\rangle_{k}
$$

Vamos mostrar que $\left(d_{3}-\delta_{3}\right)(1 \otimes p \otimes 1) \in\left\langle\overline{\mathcal{L}}_{2}^{\prec}(p)\right\rangle_{k}$. Podemos escrever $p=u_{0} u_{1} u_{2}=v_{2} v_{1} v_{0}$ como 3 -ambiguidade à esquerda e direita respectivamente. Logo,

$$
\delta_{3}(1 \otimes p \otimes 1)=\pi_{2} f_{3} i_{3}(1 \otimes p \otimes 1)=\pi\left(v_{2}\right) \otimes v_{1} v_{0} \otimes 1-1 \otimes u_{0} u_{1} \pi\left(u_{2}\right) .
$$

Agora considere $r^{p}=\left(r_{n}, \ldots, r_{1}\right)$ e $t^{p}=\left(t_{m}, \ldots, t_{1}\right)$ tais que $r^{p}$ começa à esquerda de $p$ e $t^{p}$ começa a direita de $p$, então

$$
\left(d_{3}-\delta_{3}\right)(1 \otimes p \otimes 1)=\phi_{2}\left(t^{\prime}, t_{1}(p)\right)-\phi_{2}\left(r^{\prime} p, r_{1}(p)\right),
$$

onde $t^{\prime p}=\left(t_{m}, \ldots, t_{1}\right)$ e $r^{\prime p}=\left(r_{n}, \ldots, r_{1}\right)$. Pelo Lema 19. $\phi_{2}\left(t^{\prime} p, t_{1}(p)\right)$ e $\phi_{2}\left(r^{\prime} p, r_{1}(p)\right)$ pertencem a $\left\langle\overline{\mathcal{L}}_{2}^{\prec}(p)\right\rangle_{k}$ e fica demonstrado o resultado.

\subsection{Contra exemplo da questão de Happel}

Seja uma álgebra $A$ sobre um corpo $k$. Se $A$ tem dimensão global finita então $H H^{*}(A)=$ $\bigoplus_{i \geq 0} H H^{i}(A)$ é uma $k$-álgebra de dimensão finita. Em 1989 Happel questiona a validade da implicação contrária para álgebras de dimensão finita, isto é, o fato de $H H^{*}(A)$ ser de dimensão finita implica a álgebra $A$ ser de dimensão global finita? [Hap89]. A resposta para esta questão é negativa e o primeiro contra exemplo foi dado por Buchweitz, Green, Madsen e Solberg em [BGMS05] em 2005, onde mostram que dado um corpo $k$, e $\xi \in k$ a álgebra $A=k\langle x, y\rangle /\left(x^{2}, y^{2}, y x-\xi x y\right)$ não tem dimensão global finita mas $H H^{*}(A)$ tem dimensão finita se $\xi$ é não nulo e não é uma raiz da unidade. Nesta seção usamos a resolução projetiva desenvolvida no presente capítulo para mostrar que $H H^{*}(A)$ tem dimensão finita. 
Seja $k$ um corpo, $\xi$ um elemento do corpo $k$ e seja $A$ a $k$-álgebra com geradores $x$ e $y$, os quais satisfazem as relações $x^{2}=0=y^{2}, y x=\xi x y$. Escolha a ordem $x<y$ e a função peso $w$ tal que $w(x)=w(y)=1$ e fixe o sistema de redução $\mathcal{R}=\left\{\left(x^{2}, 0\right),\left(y^{2}, 0\right),(y x, \xi x y)\right\}$, o qual mostramos no Exemplo 13. que satisfaz a condição do Diamante para $I=\left\langle x^{2}, y^{2}, y x-\xi x y\right\rangle$. No Exemplo 15 vimos que para todo $n$, o conjunto $\mathcal{A}_{n}$ está dado por

$$
\mathcal{A}_{n}=\left\{y^{s} x^{t} \mid s+t=n\right\} .
$$

A Proposição 18. fornece o começo duma resolução projetiva de $A$

$$
A \otimes_{E} k \mathcal{A}_{3} \otimes_{E} A \stackrel{d_{3}}{\rightarrow} A \otimes_{E} k \mathcal{A}_{2} \otimes_{E} A \stackrel{d_{2}}{\rightarrow} A \otimes_{E} k \mathcal{A}_{1} \otimes_{E} A \stackrel{d_{1}}{\rightarrow} A \otimes_{E} A \stackrel{d_{0}}{\rightarrow} A \rightarrow 0
$$

onde $d_{2}$ é a aplicação de $A$-bimódulos tal que

$$
\begin{aligned}
& d_{2}\left(1 \otimes x^{2} \otimes 1\right)=x \otimes x \otimes 1+1 \otimes x \otimes x \\
& d_{2}\left(1 \otimes y^{2} \otimes 1\right)=y \otimes y \otimes 1+1 \otimes y \otimes y \\
& d_{2}(1 \otimes y x \otimes 1)=y \otimes x \otimes 1+1 \otimes y \otimes x-\xi x \otimes y \otimes 1-\xi \otimes x \otimes y
\end{aligned}
$$

e $d_{3}$ é a aplicação de $A$-bimódulos tal que

$$
\begin{aligned}
d_{3}\left(1 \otimes y^{3} \otimes 1\right) & =y \otimes y^{2} \otimes 1-1 \otimes y^{2} \otimes y \\
d_{3}\left(1 \otimes y^{2} x \otimes 1\right) & =y \otimes y x \otimes 1+\xi \otimes y x \otimes y+\xi^{2} x \otimes y^{2} \otimes 1-1 \otimes y^{2} \otimes x \\
d_{3}\left(1 \otimes y x^{2} \otimes 1\right) & =y \otimes x^{2} \otimes 1-1 \otimes y x \otimes x-\xi x \otimes y x \otimes 1-\xi^{2} \otimes x^{2} \otimes y \\
d_{3}\left(1 \otimes x^{3} \otimes 1\right) & =x \otimes x^{2} \otimes 1-1 \otimes x^{2} \otimes x
\end{aligned}
$$

Dado $q \in \mathcal{A}_{n}$, existem $s, t \in \mathbb{N}$ tais que $s+t=n$ e $q=y^{s} x^{t}$. Os únicos divisores de $q$ em $\mathcal{A}_{n-1}$ são $y^{s-1} x^{t}$ e $y^{s} x^{t-1}$ e portanto $\delta_{n}: A \otimes_{E} k \mathcal{A}_{n} \otimes_{E} A \rightarrow A \otimes_{E} k \mathcal{A}_{n-1} \otimes_{E} A$ está definido por

$$
\delta_{n}\left(1 \otimes y^{s} x^{t} \otimes 1\right)= \begin{cases}y \otimes y^{s-1} x^{t} \otimes 1+(-1)^{n} \otimes y^{s} x^{t-1} \otimes x, & \text { se } s \neq 0 \text { e } t \neq 0, \\ y \otimes y^{n-1} \otimes 1+(-1)^{n} \otimes y^{n-1} \otimes y & \text { se } t=0, \\ x \otimes x^{n-1} \otimes 1+(-1)^{n} \otimes x^{n-1} \otimes x & \text { se } s=0,\end{cases}
$$

Por definição

$$
\overline{\mathcal{L}}_{n-1}^{\prec}\left(y^{s} x^{t}\right)=\left\{\lambda \pi(b) \otimes p \otimes \pi\left(b^{\prime}\right) \mid b, b^{\prime} \in \mathcal{B}, p \in \mathcal{A}_{n-1}, \lambda b p b^{\prime} \prec y^{s} x^{t}\right\},
$$

$\lambda b p b^{\prime} \prec y^{s} x^{t}$ se existe uma redução $r$ tal que $r\left(y^{s} x^{t}\right)=\lambda b p b^{\prime}+x$, com $b p b^{\prime} \notin \operatorname{supp}(x)$. Como $S=\left\{x^{2}, y^{2}, y x\right\}$, toda redução básica $r$ tal que $r\left(y^{s} x^{t}\right) \neq y^{s} x^{t}$ satisfaz $r\left(y^{s} x^{t}\right)=0$ ou $r\left(y^{s} x^{t}\right)=\xi y^{s-1} x y x^{t-1}$.

Se $s>0, t>0$ então

$$
\overline{\mathcal{L}}_{n-1}^{\prec}\left(y^{s} x^{t}\right)=\left\{\xi^{s} x \otimes y^{s} x^{t-1} \otimes 1, \xi^{t} \otimes y^{s-1} x^{t} \otimes y\right\},
$$

Se $s=0$ ou $t=0$ o conjunto $\overline{\mathcal{L}}_{n-1}^{\prec}\left(y^{s} x^{t}\right)$ é vazio.

Consideremos o complexo

$$
\cdots \stackrel{d_{n+1}}{\longrightarrow} A \otimes_{E} k \mathcal{A}_{n} \otimes_{E} A \stackrel{d_{n}}{\longrightarrow} \cdots \stackrel{d_{2}}{\longrightarrow} A \otimes_{E} k \mathcal{A}_{1} \otimes_{E} A \stackrel{d_{1}}{\longrightarrow} A \otimes_{E} A \stackrel{d_{0}}{\longrightarrow} A \rightarrow 0
$$

onde

$$
\begin{aligned}
d_{n}\left(1 \otimes y^{s} x^{t} \otimes 1\right) & =y \otimes y^{s-1} x^{t} \otimes 1+(-1)^{n} \otimes y^{s} x^{t-1} \otimes x \\
& +(-1)^{s} \xi^{s} x \otimes y^{s} x^{t-1} \otimes 1+(-1)^{s} \xi^{t} \otimes y^{s-1} x^{t} \otimes y
\end{aligned}
$$


para $s>0$ e $t>0$ e

$$
\begin{aligned}
& d_{n}\left(1 \otimes y^{n} \otimes 1\right)=y \otimes y^{n-1} \otimes 1+(-1)^{n} 1 \otimes y^{n-1} \otimes y, \\
& d_{n}\left(1 \otimes x^{n} \otimes 1\right)=x \otimes x^{n-1} \otimes 1+(-1)^{n} 1 \otimes x^{n-1} \otimes x .
\end{aligned}
$$

Este complexo satisfaz as condições do Teorema(13), portanto é uma resolução projetiva de bimódulos de $A$. Aplicando o funtor $\operatorname{Hom}_{A^{e}}(-, A)$ a (3.8) e usando o isomorfismo

$$
\operatorname{Hom}_{A^{e}}\left(A \otimes_{E} k \mathcal{A}_{n} \otimes_{E} A, A\right) \cong \operatorname{Hom}_{E-E}\left(k \mathcal{A}_{n}, A\right),
$$

obtemos o complexo

$$
\begin{aligned}
0 \rightarrow & \operatorname{Hom}_{E-E}\left(k \mathcal{A}_{0}, A\right) \stackrel{\phi_{1}}{\longrightarrow} \operatorname{Hom}_{E-E}\left(k \mathcal{A}_{1}, A\right) \stackrel{\phi_{2}}{\longrightarrow} \operatorname{Hom}_{E-E}\left(k \mathcal{A}_{2}, A\right) \stackrel{\phi_{3}}{\longrightarrow} \cdots \\
& \quad \cdots \rightarrow \operatorname{Hom}_{E-E}\left(k \mathcal{A}_{n-1}, A\right) \stackrel{\phi_{n}}{\longrightarrow} \operatorname{Hom}_{E-E}\left(k \mathcal{A}_{n}, A\right) \stackrel{\phi_{n+1}}{\longrightarrow} \operatorname{Hom}_{E-E}\left(k \mathcal{A}_{n+1}, A\right) \rightarrow \cdots
\end{aligned}
$$

onde

$$
\phi_{n}(f)\left(y^{s} x^{t}\right)=y f\left(y^{s-1} x^{t}\right)+(-1)^{n} f\left(y^{s} x^{t-1}\right) x+(-1)^{s} \xi^{s} x f\left(y^{s} x^{t-1}\right)+(-1)^{s} \xi^{t} f\left(y^{s-1} x^{t}\right) y,
$$

para todo $f \in H_{\text {om }}\left(k \mathcal{A}_{n-1}, A\right)$ e $s>0, t>0$ tais que $s+t=n$, e

$$
\begin{aligned}
& \phi_{n}(f)\left(y^{n}\right)=y f\left(y^{n-1}\right)+(-1)^{n} f\left(y^{n-1}\right) y, \\
& \phi_{n}(f)\left(x^{n}\right)=x f\left(x^{n-1}\right)+(-1)^{n} f\left(x^{n-1}\right) x,
\end{aligned}
$$

O $i$-ésimo grupo de cohomologia de Hochschild de $A$ fica determinado por

$$
H H^{i}(A)=\operatorname{ker}\left(\phi_{n+1}\right) / \operatorname{Im}\left(\phi_{n}\right) \text {. }
$$

Vamos calcular os primeiros grupos de cohomologia e mostrar que para o caso em que $\xi$ é não nulo e não é uma raiz da unidade $H H^{n}(A)=0$ para $n \geq 3$. Estes resultados coincidem com o feito em [BGMS05].

Seja $g \in \operatorname{Hom}_{E-E}\left(k \mathcal{A}_{0}, A\right)$ e $\alpha \in\{x, y\}$, então $\phi_{1}(g)(\alpha)=\alpha g(v)-g(v) \alpha$. Logo, se $g \in k e r\left(\phi_{1}\right)$ devemos ter

$$
\begin{array}{r}
g(v)=g(v) x \\
y g(v)=g(v) x
\end{array}
$$

Como $g(v) \in A$ podemos expressar ele da forma $g(v)=\lambda_{1}+\lambda_{2} x+\lambda_{3} y+\lambda_{4} x y$. As igualdades anteriores implicam $\lambda_{3} x y=\lambda_{3} \xi x y$ e $\lambda_{2} \xi x y=\lambda_{2} x y$. Logo, $\lambda_{3}(1-\xi)=0$ e $\lambda_{2}(\xi-1)=0$. Portanto

$$
H H^{0}(A)= \begin{cases}A & \text { se } \xi=1 \\ \operatorname{span}_{k}\{1, x y\} & \text { se } \xi \neq 1\end{cases}
$$

Seja $g \in H_{o m} m_{E}\left(k \mathcal{A}_{1}, A\right)$, tal que $g \in \operatorname{ker}\left(\phi_{2}\right)$. Então

$$
\begin{aligned}
\phi_{2}(g)\left(y^{2}\right) & =y g(y)+g(y) y=0, \\
\phi_{2}(g)(y x) & =y g(x)+g(y) x-\xi x g(y)-\xi g(x) y=0, \\
\phi_{2}(g)\left(x^{2}\right) & =x g(x)+g(x) x=0 .
\end{aligned}
$$

Escrevendo $g(x)$ e $g(y)$ da forma $g(x)=\lambda_{1}+\lambda_{2} x+\lambda_{3} y+\lambda_{4} x y$ e $g(y)=\mu_{1}+\mu_{2} x+\mu_{3} y+\mu_{4} x y$, obtemos o sistema de equações 


$$
\begin{array}{r}
2 \lambda_{1} x+\lambda_{3}(1+\xi) x y=0, \\
2 \mu_{1} y+\mu_{2}(\xi+1) x y=0, \\
\lambda_{1}(1-\xi) y+\mu(1-\xi) x=0 .
\end{array}
$$

Para calcular $H H^{1}(A)$ vamos considerar 4 casos:

CASO 1: Se $\xi \neq-1,1$, então $\lambda_{1}=\mu_{1}=\lambda_{3}=\mu_{2}=0$, logo $\operatorname{dim}_{k}\left(k e r\left(\phi_{2}\right)\right)=4$. Neste caso $H H^{0}(A)=\operatorname{ker}\left(\phi_{1}\right)=\operatorname{span}_{k}\{1, x y\}$, portanto $\operatorname{dim}_{k}\left(\operatorname{Im}\left(\phi_{1}\right)\right)=2$ e $\operatorname{dim}_{k} H H^{1}(A)=2$.

CASO 2: Se $\xi=1, \xi \neq-1,(\operatorname{char}(k) \neq 2)$ então $\lambda_{1}=\mu_{1}=\lambda_{3}=\mu_{2}=0, \operatorname{logo} \operatorname{dim}_{k}\left(\operatorname{ker}\left(\phi_{2}\right)\right)=$ 4. Neste caso $\operatorname{dim}_{k}\left(\operatorname{Im}\left(\phi_{1}\right)\right)=0$ e portanto $\operatorname{dim}_{k} H H^{1}(A)=4$.

CASO 3: Se $\xi \neq 1, \xi=-1,(\operatorname{char}(k) \neq 2)$ então $\lambda_{1}=\mu_{1}=0, \operatorname{logo}_{\operatorname{dim}}\left(k e r\left(\phi_{2}\right)\right)=6$. Neste caso $\operatorname{dim}_{k}\left(\operatorname{Im}\left(\phi_{1}\right)\right)=2$ e portanto $\operatorname{dim}_{k} H H^{1}(A)=4$.

CASO 4: Se $\xi=1$ e $\operatorname{char}(k)=2$ então $\operatorname{dim}_{k} H H^{1}(A)=8$ e $\operatorname{dim}_{k} \operatorname{Im}\left(\phi_{1}\right)=0$, portanto $\operatorname{dim}_{k} H H^{1}(A)=8$.

Sumarizando os resultados anteriores temos

$$
\operatorname{dim}_{k} H H^{1}(A)= \begin{cases}2 & \text { se } \xi \neq 1,-1 \\ 4 & \text { se }(\xi=1 \text { e } \xi \neq-1) \text { ou }(\xi \neq 1 \text { e } \xi=-1) \\ 8 & \text { se } \xi=-1 \text { e } \operatorname{char}(k)=2 .\end{cases}
$$

Fazendo um processo análogo ao feito para calcular a 0-ésima cohomologia e a dimensão da primeira cohomologia é possível obter a dimensão da segunda cohomologia. De fato

$$
\operatorname{dim}_{k} H H^{2}(A)= \begin{cases}1 & \text { se } \xi \neq 1,-1, \\ 5 & \text { se } \xi=1 \text { e } \xi \neq-1, \\ 6 & \text { se } \xi \neq 1 \text { e } \xi=-1, \\ 10 & \text { se } \xi=-1 \text { e } \operatorname{char}(k)=2 .\end{cases}
$$

Podemos supor sem perda de generalidade $n>3$ par e $f \in H_{o m-E}\left(k \mathcal{A}_{n}, A\right)$. O valor de $f$ nas $n$-ambiguidades pode ser expresso por

$$
f\left(y^{n-i} x^{i}\right)=\lambda_{1}^{(n-i)(i)}+\lambda_{2}^{(n-i)(i)} x+\lambda_{3}^{(n-i)(i)} y+\lambda_{4}^{(n-i)(i)} x y
$$

para todo $i=0,1, \ldots, n$. Se $f \in \operatorname{ker}\left(\phi_{n+1}\right)$ as seguintes igualdades devem ser satisfeitas para todos os pares $s$ e $t$ tais que $s+t=n+1$ e $s=1,2, \ldots, n$

$$
\begin{aligned}
\lambda_{2}^{(n)(0)}(\xi-1) & =0, \\
\lambda_{1}^{(s-1)(t)}\left(1+(-1)^{s} \xi^{t}\right) & =0, \\
\lambda_{1}^{(s)(t-1)}\left((-1)^{s} \xi^{s}-1\right) & =0, \\
\lambda_{3}^{(0)(n)}(1-\xi) & =0,
\end{aligned}
$$

$\mathrm{e}$

$$
\xi \lambda_{2}^{(s-1)(t)}-\xi \lambda_{3}^{(s)(t-1)}+(-1)^{s} \xi^{s} \lambda_{3}^{(s)(t-1)}+(-1)^{s} \xi^{t} \lambda_{2}^{(s-1)(t)}=0 .
$$

Vamos supor que $\xi$ é não nulo e não é uma raiz da unidade. Note que isto implica $\operatorname{char}(k) \neq 2$. Com estas hipóteses e as igualdades anteriores obtemos que $\lambda_{2}^{(n)(0)}=\lambda_{3}^{(0)(n)}=0, \lambda_{1}^{(n-i)(i)}=0$ para todo $i=0,1, \ldots, n$ e $\lambda_{2}^{(i)(n-i)}$ sempre pode ser expresso em termos de $\lambda_{3}^{(i+1)(n-i-1)}$ para todo 
$i=0,1, \ldots, n-1$. Portanto

$$
\operatorname{dim}_{k} \operatorname{ker}\left(\phi_{n+1}\right)=\operatorname{dim}_{k} \operatorname{Hom}_{E-E}\left(k \mathcal{A}_{n}, A\right)-(2 n+3)=4(n+1)-(2 n+3)=2 n+1 .
$$

Para calcular a dimensão de $\operatorname{Im}\left(\phi_{n}\right)$ fixamos $f \in H_{\text {om-E }}\left(k \mathcal{A}_{n-1}, A\right)$ e expressamos a imagem das $(n-1)$-ambiguidades por

$$
f\left(y^{n-i} x^{i-1}\right)=\lambda_{1}^{(n-i)(i-1)}+\lambda_{2}^{(n-i)(i-1)} x+\lambda_{3}^{(n-i)(i-1)} y+\lambda_{4}^{(n-i)(i-1)} x y
$$

para todo $i=1,2, \ldots, n$. Neste caso as igualdades a serem satisfeitas pelos coeficientes na expressão de $f\left(y^{n-i} x^{i-1}\right)$ e por $\xi$ são

$$
\begin{aligned}
2 \lambda_{1}^{(n-1)(0)} & =0, & & \lambda_{2}^{(n-1)(0)}(\xi+1)=0, \\
\lambda_{1}^{(s-1)(t)}\left(1+(-1)^{s} \xi^{t}\right) & =0, & & \lambda_{1}^{(s)(t-1)}\left(1+(-1)^{s} \xi^{s}\right)=0, \\
2 \lambda_{1}^{(0)(n-1)} & =0, & & \lambda_{3}^{(0)(n-1)}(1+\xi)=0,
\end{aligned}
$$

$\mathrm{e}$

$$
\xi \lambda_{2}^{(s-1)(t)}+\xi \lambda_{3}^{(s)(t-1)}+(-1)^{s} \xi^{s} \lambda_{3}^{(s)(t-1)}+(-1)^{s} \xi^{t} \lambda_{2}^{(s-1)(t)}=0 .
$$

Note que em (3.10) se $s=1$ então $\lambda_{2}^{(s-1)(t)}=\lambda_{2}^{(0)(n-1)}=0$ e se $t=1$ então $\lambda_{3}^{(s)(t-1)}=\lambda_{3}^{(n-1)(0)}$. Para $s>0, t>0 \lambda_{2}^{(s-1)(t)}$ pode ser expresso em termos de $\lambda_{3}^{(s)(t-1)}$. Como $\xi$ é não nulo e não é uma raiz da unidade temos que $\lambda_{1}^{(n-1)(0)}=\lambda_{2}^{(n-1)(0)}=\lambda_{1}^{(0)(n-1)}=\lambda_{3}^{(0)(n-1)}=0$ e $\lambda_{1}^{(n-i)(i-1)}=0$ para todo $i=1,2, \ldots, n$. Isto implica $\operatorname{dim}_{k} \operatorname{Im}\left(\phi_{n}\right)=2 n+1=\operatorname{dim}_{k} k e r\left(\phi_{n+1}\right)$.

Usando os resultados obtidos para os primeiros grupos de cohomologia de $A$ e assumindo $\xi$ não nulo e $\xi^{l} \neq 1$ para todo $l \geq 1$, temos que $\operatorname{dim}_{k} H H^{0}(A)=2, \operatorname{dim}_{k} H H^{1}(A)=2, \operatorname{dim}_{k} H H^{2}(A)=1$ e $\operatorname{dim}_{k} H H^{n}(A)=0$ para todo $n \geq 3$. Portanto,

$$
\operatorname{dim}_{k} H H^{*}(A)=5 .
$$




\section{Projetos para o futuro}

Usando os resultados que consegui no meu trabalho de graduação [LP18], junto com os resultados de Chohuy e Bardzell pretendemos distinguir classes de álgebras importantes e suas categorias derivadas. No meu doutorado pretendo fazer um curso de categorias derivadas para esse fim e me parece que as álgebras Calabi-Yau e as de $d$-Koszul, [GMMVZ04] podem ser as primeiras a serem atacadas. Isso parece interessante também porque recentemente meu orientador e o Prof. Yury Volkov, classificaram as álgebras $d$-homogêneas cujo ideal definidor pode ser gerado por no máximo dois elementos[MV17, MV]. Uma outra direção possível seria estudar a geometria por trás das bases de Gröbner. Pretendo estudar esse tópico baseado nos trabalhos de Green, Schroll [GHS17] bem como o pre print do meu orientador com Green e Schroll [GMS17]. 


\section{Referências Bibliográficas}

[AF12] F. W. Anderson e K. R. Fuller. Rings and categories of modules, volume 13. Springer Science \& Business Media, 2012. 3

[AG87] D. Anick e E. L. Green. On the homology of quotients of path algebras. Communications in Algebra, 15(1-2):309-341, 1987. iii, v, 1

[Ani86] D. Anick. On the homology of associative algebras. Transactions of the American Mathematical Society, 296(2):641-659, 1986. 1

[ARS97] M. Auslander, I. Reiten e S. Smalo. Representation theory of Artin algebras, volume 36. Cambridge university press, 1997. 10

[ASS06] I. Assem, A. Skowronski e D. Simson. Elements of the Representation Theory of Associative Algebras: Volume 1: Techniques of Representation Theory, volume 65. Cambridge University Press, 2006. 3, 18

[Bar97] M. J. Bardzell. The alternating syzygy behavior of monomial algebras. Journal of Algebra, 188(1):69-89, 1997. iii, v, 1, 27, 34, 36, 37

[BGMS05] R.-O. Buchweitz, E. L. Green, D. Madsen e Ø. Solberg. Finite Hochschild cohomology without finite global dimension. Math. Res. Lett., 12(5-6):805-816, 2005. 45, 47

[BLM00] M. J. Bardzell, A. C. Locateli e E. N. Marcos. On the Hochschild cohomology of truncated cycle algebras. Comm. Algebra, 28(3):1615-1639, 2000. 21

[CE16] H. Cartan e S. Eilenberg. Homological Algebra (PMS-19), volume 19. Princeton University Press, 2016. 1, 14

[Cib90] C. Cibils. Rigidity of truncated quiver algebras. Advances in Mathematics, 79(1):1842, 1990. iii, v, 1, 22

[Cib98] C. Cibils. Hochschild cohomology algebra of radical square zero algebras. Em Algebras and modules, II, volume 24, páginas 93-101. Amer. Math. Soc. Providence, RI, 1998. 23

[CLS82] C. Cibils, F. Larrión e L. Salmerón. Métodos diagramáticos en teoría de representaciones. Monografías del Instituto de Matemáticas. Universidad Nacional Autónoma de México, 1982. 3

[CS15] S. Chouhy e A. Solotar. Projective resolutions of associative algebras and ambiguities. Journal of Algebra, 432:22-61, 2015. iii, v, 1, 27, 33, 34, 38

[DK12] Ju. A. Drozd e V. Kirichenko. Finite dimensional algebras. Springer Science \& Business Media, 2012. 17, 18, 22

[FFG93] D. R. Farkas, C.D. Feustel e E. L. Green. Synergy in the theories of gröbner bases and path algebras. Canadian Journal of Mathematics, 45(4):727-739, 1993. 34 
[Ger63] M. Gerstenhaber. The cohomology structure of an associative ring. Annals of Mathematics, páginas 267-288, 1963. 14

[Ger64] M. Gerstenhaber. On the deformation of rings and algebras. Annals of Mathematics, páginas 59-103, 1964. 1

[GHS17] E. L. Green, L. Hille e S. Schroll. Algebras and varieties. ArXiv e-prints, Julho 2017. 51

[GHZ $\left.{ }^{+} 85\right]$ E. L. Green, D. Happel, D. Zacharia et al. Projective resolutions over artin algebras with zero relations. Illinois Journal of Mathematics, 29(1):180-190, 1985. 34

[GMMVZ04] E. L. Green, E. N. Marcos, R. Martmez-Villa e P. Zhang. D-koszul algebras. Journal of Pure and Applied Algebra, 193(1-3):141-162, 2004. 51

[GMS17] E. L. Green, E. N. Marcos e S. Schroll. Module and varieties. arXiv:170\%.07877, Julho 2017. 51

[Gro91] Buenos Aires Cyclic Homology Group. Cyclic homology of algebras with one generator. K-theory, 5:51-69, 1991. 15

[Hap89] D. Happel. Hochschild cohomology of finite - dimensional algebras. Em Marie-Paule Malliavin, editor, Séminaire d'Algèbre Paul Dubreil et Marie-Paul Malliavin, páginas 108-126, Berlin, Heidelberg, 1989. Springer Berlin Heidelberg. iii, v, 1, 16, 20, 21, 25, 45

[Hoc45] G. Hochschild. On the cohomology groups of an associative algebra. Annals of Mathematics, páginas 58-67, 1945. 1, 12, 18

[LP18] O. Lezama e M. Paiba. Computing finite presentations of Tor and Ext skew PBW extensions and some applications. Acta Math. Acad.Paedagog. Nyházi, 34(1), 2018. 51

[MV] E. N. Marcos e Y. Volkov. s-homogeneous algebras with two relations. Manuscript in preparation. 51

[MV17] E. N. Marcos e Y. Volkov. s-homogeneous algebras via $s$-homogeneous triples. arXiv preprint arXiv:1711.10664, 2017. 51

[Red01] M. J. Redondo. Hochschild cohomology: some methods for computations. Resenhas do Instituto de Matemática e Estatística da Universidade de São Paulo, 5(2):113-137, 2001. 14,18

[Skö08] E. Sköldberg. A contracting homotopy for bardzell's resolution. Em Mathematical Proceedings of the Royal Irish Academy, páginas 111-117. JSTOR, 2008. 38

[Wei95] C. Weibel. An introduction to homological algebra. Number 38. Cambridge university press, 1995. 3, 14 